\title{
THE SECOND-GENERATION GUIDE STAR CATALOG: DESCRIPTION AND PROPERTIES
}

\author{
Barry M. Lasker ${ }^{1,10}$, Mario G. Lattanzi ${ }^{2}$, Brian J. McLean ${ }^{1}$, \\ Beatrice Bucciarelli ${ }^{2}$, Ronald Drimmel ${ }^{2}$, Jorge Garcia ${ }^{5}$, Gretchen Greene ${ }^{1}$, Fabrizia \\ Guglielmetti ${ }^{6}$, Christopher Hanley ${ }^{1}$, George Hawkins ${ }^{1}$, Victoria G. Laidler ${ }^{1,3}$, Charles \\ Loomis ${ }^{1,3}$, Michael Meakes ${ }^{1}$, Roberto Mignani ${ }^{7}$, Roberto Morbidelli ${ }^{2}$, Jane Morrison ${ }^{4}$, \\ Renato Pannunzio ${ }^{2}$, Amy Rosenberg ${ }^{1}$, Maria Sarasso ${ }^{2}$, Richard L. Smart ${ }^{2}$, Alessandro \\ Spagna ${ }^{2}$, Conrad R. Sturch ${ }^{1,3,9}$, Antonio Volpicelli ${ }^{2}$, Richard L. White ${ }^{1}$, David Wolfe ${ }^{1}$, \\ and Andrea Zacchei ${ }^{8}$
}

\begin{abstract}
The Guide Star Catalog II (GSC-II) is an all-sky database of objects derived from the uncompressed Digitized Sky Surveys that the Space Telescope Science Institute has created from the Palomar and UK Schmidt survey plates and made available to the community.

Like its predecessor (GSC-I), the GSC-II was primarily created to provide guide star information and observation planning support for Hubble Space Telescope. This version, however, is already employed at some of the ground-based new-technology telescopes such as GEMINI, VLT, and TNG, and will also be used to provide support for the James Webb Space Telescope (JWST) and Gaia space missions as well as the Large Sky Area Multi-Object Fiber Spectroscopic Telescope, one of the major ongoing scientific projects in China.

Two catalogs have already been extracted from the GSC-II database and released to the astronomical community. A magnitude-limited $\left(R_{F}=18.0\right)$ version, GSC2.2, was distributed soon after its production in 2001, while the GSC2.3 release has been available for general access since 2007.

The GSC2.3 catalog described in this paper contains astrometry, photometry, and classification for $945,592,683$ objects down to the magnitude limit of the plates. Positions are tied to the International Celestial Reference System; for stellar sources, the all-sky average absolute error per coordinate ranges from $0 . .^{\prime \prime} 2$ to $0 .{ }^{\prime \prime} 28$ depending on magnitude. When dealing with extended objects, astrometric errors are $20 \%$ worse in the case of galaxies and approximately a factor of 2 worse for blended images.

Stellar photometry is determined to $0.13-0.22 \mathrm{mag}$ as a function of magnitude and photographic passbands $\left(R_{F}, B_{J}, I_{N}\right)$. Outside of the galactic plane, stellar classification is reliable to at least $90 \%$ confidence for magnitudes brighter than $R_{F}=19.5$, and the catalog is complete to $R_{F}=20$.
\end{abstract}

Subject headings: astrometry - astronomical data bases: miscellaneous - catalogs - surveys - techniques: image processing - photometric

\footnotetext{
${ }^{1}$ Space Telescope Science Institute, 3700 San Martin Drive, Baltimore, MD 21218, USA

${ }^{2}$ INAF-Osservatorio Astronomico di Torino, Strada Osservatorio 20, 10025 Pino Torinese, TO, Italy

${ }^{3}$ Computer Sciences Corporation, Space Telescope Sci-
}

\footnotetext{
ence Institute, 3700 San Martin Drive, Baltimore, MD 21218, USA

${ }^{4}$ Steward Observatory, 9425 N.Weather Hill Dr., Tucson, AZ 5743-5484, USA

${ }^{5}$ Gemini Observatory Southern Operations Center, c/o
} 


\section{Introduction}

The Guide Star Catalog II (GSC-II) is an astronomical database constructed from the scanned images of 9541 Palomar and UK Schmidt photographic sky survey plates digitized at Space Telescope Science Institute (STScI). These same plate images are also known as the Digitized Sky Survey (DSS), and are accessible directly from the STScI eb site as well as from a number of major astronomical data centers around the world. A subset of the original images, based on the second-epoch Palomar surveys only (also known as DPOSS), is available separately from Caltech (Djorgovski et al. 2003). Whilst all the images represent over 8 terabytes of data that are archived at STScI, the use of the H-transform compression technique (White et al. 1992; White and Percival 1994) has enabled a reduction in data volume to $\approx 1$ terabyte for distribution to data centers.

Each individual uncompressed image was processed to detect the objects, and calibrations were obtained for each plate by polynomial modeling against photometric and astrometric reference catalogs. The average accuracy in position for stellar objects of intermediate magnitude $(V \lesssim 18.5)$ is $\sim 0.3^{\prime \prime}$, whilst the corresponding astrometric precision, on a scale of approximately 0.5 degrees, is close to $0.2^{\prime \prime}$. Photometric calibrations, carried out in the natural plate passband, show typical errors of 0.1-0.2 mag and systematic offsets lower than 0.1 mag. Stars are correctly classified as such with at least $90 \%$ confidence for magnitudes brighter than $R_{F}=19.5$.

The plate catalogs were loaded into a database system known as Catalog of Objects and Measured Parameters from All Sky Surveys (COMPASS), (see Lasker et al. 1998). This database was built using "Objectivity", a commercial ObjectOriented Database Management System; it consti-

AURA, Casilla 603, La Serena, Chile

${ }^{6}$ Max-Planck-Institut fr Plasmaphysik, Ber:MF Geb:L4 Zi:338, Boltzmannstrasse 2, 85748 Garching bei Muenchen, Germany

${ }^{7}$ Mullard Space Science Laboratory, University College London, Holmbury St. Mary, Dorking, Surrey, RH5 6NT, $\mathrm{UK}$

${ }^{8}$ Osservatorio Astronomico di Trieste, Via Tiepolo 11, 34131, Trieste, Italy

${ }^{9}$ Retired

${ }^{10}$ Deceased tutes a repository for $\approx 5$ billion measurements, and is $\approx 2.5$ terabytes in size. Once the individual observations have been cross-matched to link different measurements of unique astronomical objects, a catalog can be exported from the database as a collection of FITS binary tables. Then, the export catalog is made available to the community via Web services and also provided to a number of data centers. Over the last few years there have been a number of interim releases from the GSC-II, primarily to consortium members for telescope operations. The latest public release, the GSC 2.3.2, contains $945,592,683$ unique objects and is $\approx 170 \mathrm{~GB}$ in size. Details about the release content and access methods are available from the ST ScI and Osservatorio Astronomico di TOrino (OATo) Web sites.

In this paper we describe the construction, calibration, and overall quality of GSC 2.3.2, the current catalog release (for simplicity, GSC 2.3 in the rest of this paper), which was investigated through internal tests and a number of external comparisons to suitable literature data.

\subsection{Background}

In the age of new-technology telescopes and space-based missions, it is useful to recall that an important part of observational astronomy has historically been the creation of catalogs containing the reference and (often) target objects required to support observing programs. From a historical viewpoint it is fascinating that astrometry - the oldest branch of astronomy - is so vital to the success of modern high-tech observatories. As the technological complexity (and cost) of building and operating telescopes has increased enormously over the last 20 years, so has the effort to provide the best scientific return for these investments. One of the important issues is the optimization of observing efficiency, which depends on the use of proper pointing and input catalogs as well as on the access to digitized versions of the optical sky surveys.

The need for a deeper, all-sky catalog was highlighted early by the creation of the first Guide Star Catalog (GSC-I) to satisfy the pointing requirements of the Hubble Space Telescope (HST). An in-depth description of the GSC-I catalog may be found in a set of three papers (Lasker et al. 1990; Russell et al. 1990; Jenkner et al. 1990). 
The GSC-I was used for HST observation planning as well as target acquisition and tracking and has proved to be very reliable for its intended purpose. In addition, since its publication on CDROM, this catalog had become widely used by many ground-based telescopes to speed up the process of finding guide stars.

Although the GSC-I has been used with great success operationally and scientifically, it became clear (even during its construction) that it was possible to improve its usefulness by addressing the known systematic calibration errors. Indeed, it was clear that an increase in scope to include multicolor, multi-epoch data would eventually lead to the requirements for a new much improved archive, the GSC-II. In addition to the risk of damaging the UV-sensitive MAMA detectors in the second generation of HST instruments by $\mathrm{O}$ stars as faint as 19th magnitude, it was generally acknowledged that the proper motions of the guide stars during the proposed lifetime of $H S T$ would pose a potential problem for the accurate pointing of the telescope during the latter years of its operation. It was also realized that a fainter archive with multicolors such as the GSC-II would address a number of other astronomical needs such as: supporting adaptive optics on the next generation of large telescopes, remote or queue scheduling capabilities, and improved detector instruments requiring color information of the target objects.

In order to be prepared for this, STScI negotiated access to the original plates of the Palomar Observatory Sky Survey (POSS-II) and, in partnership with the Anglo-Australian Observatory (AAO), undertook the Second Epoch Southern (SES) red survey with the UK Schmidt Telescope Unit (UKSTU). These new surveys, when combined with the plate material used in the GSCI and the earlier POSS-I and UKSTU surveys, provide the material to generate a GSC-II based on, at least, two epochs and three passbands.

\subsection{The GSC-II Project}

STScI and OATo first began a collaboration to start developing GSC-II in 1989. STScI was primarily motivated by telescope operations, and OATo was interested in the scientific applications of this catalog for galactic structure.

Once the project was underway, additional re- sources were contributed by ESO and GEMINI (who wished to use GSC-II for VLT and GEMINI telescope operations respectively), ST-ECF (as part of the NASA-ESA HST funding agreement), and the Astrophysics Division of ESA (for science projects). A significant fraction $(\sim 30 \%)$ of the plate processing was performed at ST-ECF using the same pipeline software system described later.

\section{Astronomical data}

\subsection{Photographic Plates}

All of the survey plates scanned in this project were originally taken by the Palomar Schmidt telescope in California, USA, and the UK Schmidt telescope at Coonabarabran, Australia. Table 1 summarizes the main characteristics of the various plate material and a complete description of the photographic surveys can be found in Morgan (1995) and references therein.

In general, there is a strong similarity between the northern and southern surveys since they used the same photographic emulsions; however, there are small differences in some of the filters used, which result in slight differences in the transmission curves as shown in Figure 1 .

\subsection{Astrometric Reference Catalogs}

Following the recommendation of the IAU XXIII General Assembly (resolution B2), the International Celestial Reference System (ICRS)was adopted to be the reference system for the GSCII. At optical wavelengths, the ICRS is defined by the Hipparcos catalog; however, the bright Hipparcos stars are heavily saturated on Schmidt plates. On the other hand, the fainter stars $(V>10)$ of the Tycho (Perryman and ESA 1997) and Tycho2 (Høg et al. 2000) catalogs, do have measurable images on the Schmidt plates, so that each survey plate is directly tied to the ICRS through Tycho's faint end. Because the Tycho-2 catalog was not available until after early 2000, a subset of the GSC 2.3 astrometric calibrations are based on the ACT catalog (Urban et al. 1998). Slight deviations of the ACT and Tycho-2 catalogs from the ICRS system are well below our measuring error. 


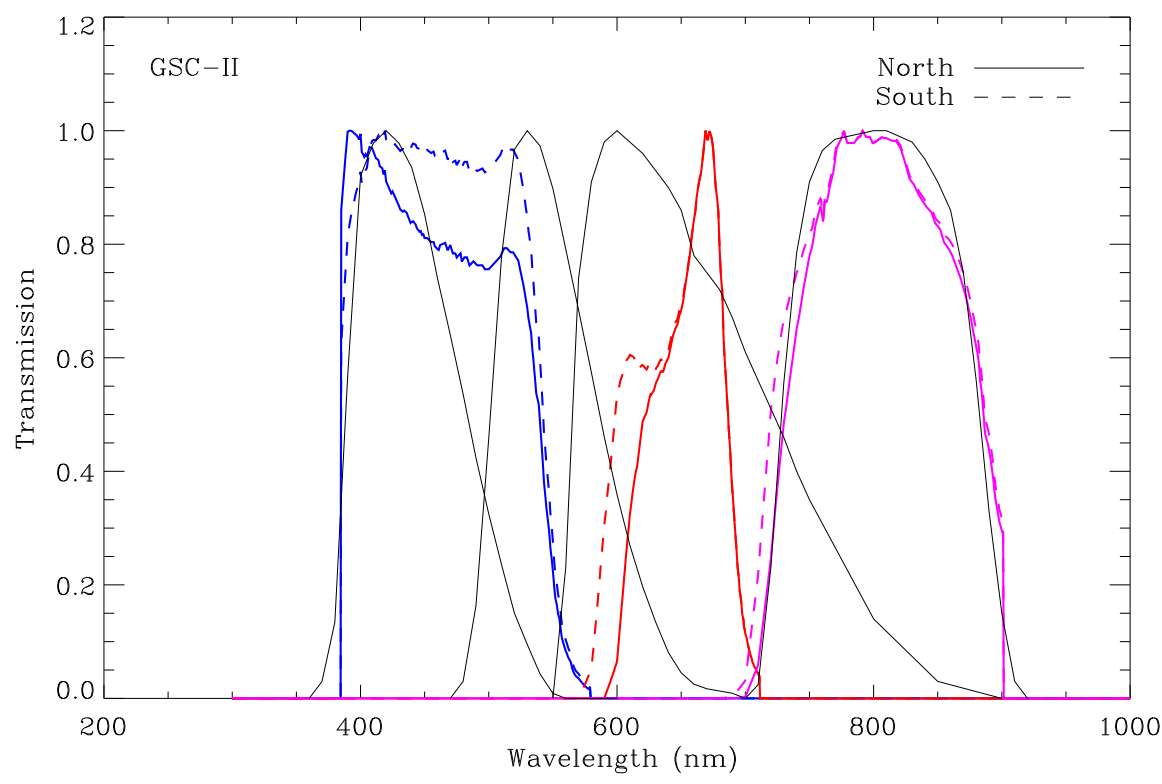

Fig. 1.- Transmission curves of the photographic passbands $B_{J}, R_{F}$, and $I_{N}$ for the Palomar (solid lines) and AAO (dashed line) Schmidt surveys, compared to the Johnson-Kron-Cousins BVRI $\mathrm{C}_{\mathrm{c}}$ filters.

\subsection{Photometric Reference Catalogs}

The photometric calibrators used for the GSCII are the GSPC-II, GSPC-I and Tycho catalogs, which sample respectively the faint, intermediate and bright range of the photographic magnitudes.

The GSPC-I (Guide Star Photometric Catalog Lasker et al. 1988), which was built to support the construction of GSC-I, contains 1477 photoelectric sequences with $B$ and $V$ standard photometry of 9-15th mag stars at the 0.1-0.15 mag level of accuracy.

Because of the nonlinear response of the photographic plate, the need for fainter calibrators was a requirement for the construction of GSCII. A lack of such calibrators in the astronomical literature motivated the start of a decade-long series of observations, only now coming to completion, which led to the construction of GSPCII (Bucciarelli et al. 2001; Bucciarelli et al. 2006). This is an all-sky catalog of CCD photometric sequences centered in each survey plate field. Each sequence provides $B, V$ and $R$ standard photometry at the 0.05-0.1 mag precision level down to approximately $\mathrm{V}=19.5$. The number of stars per sequence varies from a dozen to several hundred depending on the field of view of the CCD and the galactic latitude. At the time of GSC 2.3 calibrations, $0.5 \%$ of the plates still did not have an extended photometric sequence. In these cases, the photometric calibration relied on existing standards and deriving artificial points, as explained in Section 3.3.2. Figure 2 shows the magnitude limit distribution of all GSPC-II calibration sequences. The Tycho catalog (Perryman and ESA 1997) was used in the GSC-II photometric calibration to stabilize the bright end of the density-tointensity curve. The calibration limits of GSC 2.3 are given in Fig. 3 which shows the distribution of the faintest GSCP-II reference stars available in the Hierarchical Triangulated Mesh (HTM) regions and used for the photometric calibration of the blue $B_{J}$ and red $R_{F}$ plates.

\section{Plate processing pipeline}

\subsection{Plate digitization}

The processing of photographic plates begins with the use of microdensitometers, machines capable of measuring photographic transmission $T$. This is the fraction of light measured as a current, transmitted by the portion of the developed 
emulsion as illuminated. The PDS machines used for the digitization of GSC-II plates measure the semispecular density which is defined as the light transmitted and diffused within an angle of $<24^{\circ}$. This is then converted into photographic density $\rho$ through the relation

$$
\rho=-\log _{10} T \text {. }
$$

As $T$ can assume all values from zero (complete opaque emulsion) to 1 (perfectly transparent media), $\rho$ can vary from zero to infinity; in practice, $\rho$ usually lies in the range $0-5.0$. The Perkin-Elmer 2020G PDSs originally selected for digitizing the STScI plate collection were first modified to cope with the accuracy requirements imposed by the GSC-I project (see Lasker et al. 1990). The increase in the number of plates $(5 \times)$ to be digitized for the GSC-II and DSS projects also required a significant increase in scanning throughput. For this reason, the two scanning machines available at STScI were refurbished with entirely new critical subsystems to increase their speed without affecting their spatial and photometric capability; the improved twin digitizing devices were named GAMMA 1 and GAMMA 2 (Laidler et al. 1994).

The Guide Star Automatic Measuring MAchine (GAMMA) is a laser-illuminated multichannel scanning microdensitometer, modularly built upon the substrate of the modified PDS used in earlier STScI work.

A rebuild of the $x$ - and $y$-servos is based on an HP 5507 laser transducer system with a custom card to implement the PDS " $1 / N$ " functionality. The light source is a spatially filtered $2 \mathrm{~mW} \mathrm{HeNe}$ laser beam, expanded to $1 \mathrm{~mm}$ diameter $\left(1 / e^{2}\right.$ of total energy), moved with a $\mathrm{TeO}(2)$ acousto-optic deflector (AOD), and finally imaged on the plate as a $42 \mu \mathrm{m}$ Gaussian spot. This size gives low aliasing with the adopted $15 \mu \mathrm{m}$ sampling. The table moves in the $x$-direction at $100 \mathrm{~mm} \mathrm{~s}^{-1}$, while the AOD steps the laser beam through a small number of channels (typically 5 ) in the $y$-direction. The AOD is controlled by a frequency synthesizer operated from a digital signal processor (DSP) which cycles through a channel-to-frequency table, synchronized by pulses from the servo $1 / N$ logic. The DSP also removes y-position errors due to residual servo auto-lock disequilibrium by applying a small frequency correction to the AOD, based on a linear function of the error signal from the $y$-servo.

The light-collecting optics consists of a standard microscope, an exit slit, an integrating sphere to make the response insensitive to the channel number, and a photomultiplier. Pixel integration times are about 25-50 $\mu \mathrm{sec}$, and the data conversion is done with a 15-bit floating point analog-todigital-converter (ADC), which supplies the photometric parameter $D_{i j}=a+b \rho_{i, j}$ linearly related to the optical density measured at pixel $i, j$.

A separate photodiode to monitor the laser flicker is also provided for photometric normalization. A dedicated VAXStation with IEEE-488 and CAMAC interfaces controls each GAMMA machine. Separate programs operate each subsystem (servo, data collection, laser monitoring, AOD, console) and communicate with each other via shared memory and common event flags. This matching of the software and hardware architectures results in easily maintainable code.

Nominal performance of the scanning machines was verified on a regular basis using several numerical methods. A sample of three areas from each scan was tested using a scan-line correlation and constrained minimization to test for scan-line "shear" as a function of channel. Scans where the shear exceeded 0.1 pixel were rejected and further tests and mechanical maintenance were then performed. In general the intrapass channels showed a very high degree of correlation and shear rarely exceeded 0.05 pixel. Periodic engineering scans were also performed on a routine basis of Max Levy 2 slanted and vertical rulings in order to tune the AOD system for optimal performance.

\subsection{Image processing}

After digitization of the Schmidt plate material on the GAMMA machines, each plate scan was processed through an image processing pipeline, as detailed in the following sections.

\subsubsection{Sky Background Determination}

A single value of the sky background standard deviation is calculated for the whole plate by subdividing it into a regular grid of $0.5 \mathrm{~mm}$ cells for the estimation of local density histograms and pixels statistics. Then, the median values of each cell are computed, and a sequence of median filters is applied at different scales $(8,4,2,1$ cells $)$ to produce 

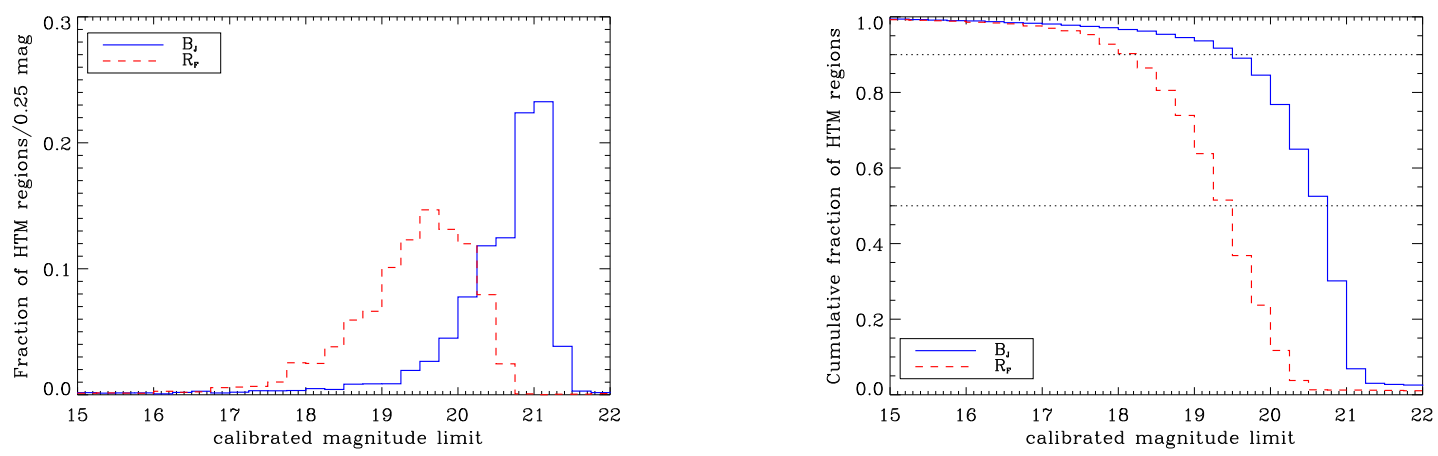

Fig. 3.- GSC 2.3 calibrated magnitude limit. Left panel: distribution of the faintest GSPC2 reference star available in the HTM regions and which result from the photometric calibration of the blue $B_{J}$ and red $R_{F}$ plates. Right panel: cumulative distribution of the HTM regions has a function of the faintest GPSC2 reference stars. Note that $\sim 90 \%$ of the sky has been calibrated with photometric sequences down to $R_{F} \gtrsim 18$ and $B_{J} \gtrsim 19.5$, while for $\sim 50 \%$ of the sky the photometric sequences attain $R_{F} \gtrsim 19.5$ and $B_{J} \gtrsim 20.5$.

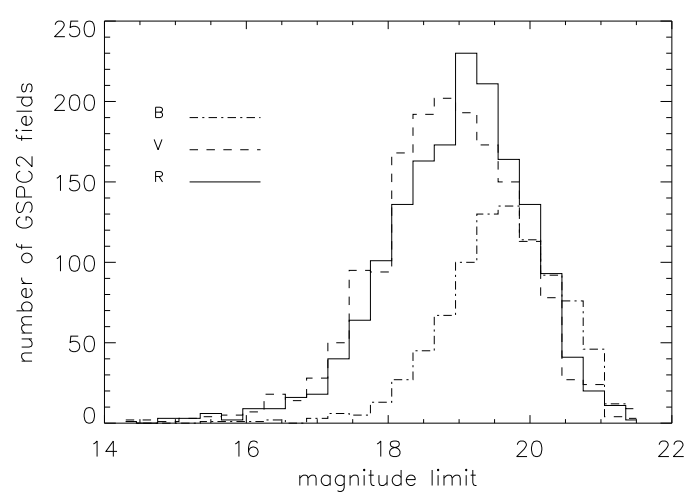

Fig. 2.- Histogram of GSPC-II sequences magnitude limit.

an average sky image. The $\sigma$ calculation for each cell is found by taking the slope of the cumulative histogram of pixel values in the cell at the 50\% level, to minimize the disturbance of stellar contribution. Pixels at this level are nearly all sky, and if the sky noise behaves as a Gaussian, the slope of the cumulative pixel histogram is $1 /(\sqrt{2 \pi} \sigma)$ which can be used to find $\sigma$ for the individual cells. The final $\sigma$ for the plate is then found from the mode of the histogram of the individual cell $\sigma$ 's. This is a good approximation for $\sigma$ over the whole plate from the unsmoothed sky using all pixels in the plate. Finally, the edges of the average sky image are examined in an attempt to avoid processing the "bad" edges of the plate as well as the label and sensitometer areas: areas in which the mean values are more than $5 \sigma$ away from the mean sky are set to a negative value, which is a flag for the object detection program to avoid processing.

\subsubsection{Object Detection}

This task used the same implementation of the ROE COSMOS image processing software used for the GSC-I (see Lasker et al. 1990; Lutz 1980; MacGillivray and Stobie 1984) which connects a minimum number of pixels that are brighter than a threshold level above the sky. The threshold level for object detection was nominally set at $3 \sigma$, though it was increased to $4 \sigma$ in cases of plates with large grain noise or in crowded fields. A single value of sigma was used for the entire plate, as described above. Fifteen additional thresholds, equally spaced (in density space) between the detection threshold and the saturation level, were also defined, and minimum areas were determined at each of these thresholds as well. A small image "cutout" was then extracted and saved around each detected object, with ample margins to include the wings of extended objects. An example of image cutouts is given in Figure 4

From the size, integrated density, and moment values of each object, parameters such as object's shape and $(x, y)$ of its centroider were derived. These and any further image processing were performed on the cutout images rather than the full 

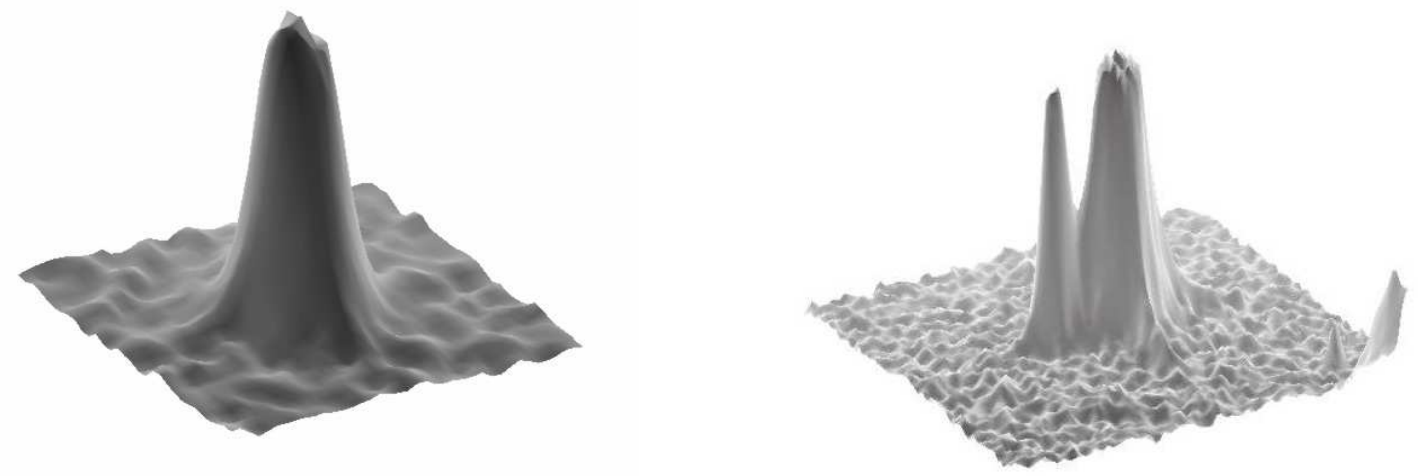

Fig. 4.- Cutout of a bright star (left panel) and of a resolved binary star (right panel). The irregular truncation of the peak of the brightest object is due to the saturation limit of the photographic emulsion.
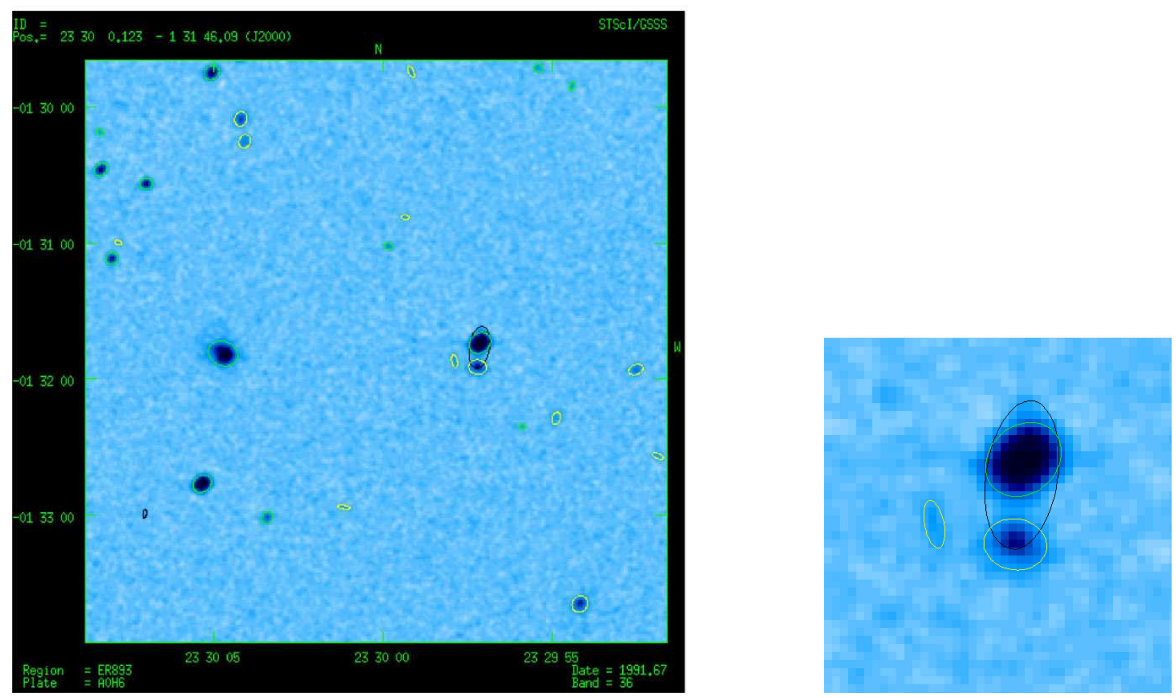

Fig. 5.- Typical high latitude field of $4^{\prime} \times 4^{\prime}$ (left panel) showing various stellar and extended objects, plus a deblended binary system (zoomed in the right panel). The identified objects are encircled by ellipses whose size and orientation are defined by the moments of their density distribution; the line color indicates the object classification: green for "stars", yellow for "nonstars", and black for either "defects" or "blends". 
scan image. The maximum cutout extracted was $4.25^{\prime} \times 4.25^{\prime}$, so, if a detected object was larger than this, it received no further processing (deblending, centroiding, etc). This results in a small number of "holes" in the GSC-II catalog around the brightest stars, galaxies, and the occasional very bright satellite trails.

\subsubsection{Blend resolution}

This is based upon the published Beard et al. 1990) ROE COSMOS deblender algorithm, which works well also in relatively crowded regions. Each new object is re-thresholded, and at each new threshold the connectivity algorithm is applied (Thanisch and Robin (1984)) to check if the object has split into two or more objects. Faint outlying pixels around blended objects are fractionally allocated according to the faint pixel allocation technique as described in these references. The deblender uses the same thresholds as the object detection task, but applies a single local sky value, i.e., the one recorded by the object detection. Once an object has been identified as single, its size, integrated density and moments are computed and parameters such as $x, y$ and shape finally calculated. An example of deblended objects formed by the two companions of a parent binary is shown in Figure 5 .

\subsubsection{Centroiding}

The centroider algorithm performs a fit to the cutout image with an elliptical Gaussian to find the object's center. After the image is normalized, the centroid is calculated iteratively, with the barycentric position as starting point, and assuming a flat sky. The centroider returns the amplitude, $x, y$, and their covariance matrix, as well as the $x$ slope, $y$ slope, and level of the fitted background. Deblended images occurring in the same cutout require special handling. First, an attempt is made to perform a simultaneous fit to all objects in the deblended family. If this fails to converge, each deblended component is run individually through the single centroider after applying the fractional pixel allocation determined by the deblender. If the centroider failed to converge on an object after a reasonable number of iterations, or if the centroider failed for some other reason, only the barycentric position determined from the object detection or deblending was used in the calibration. If the object had been classified as a plate artifact, which has a very low chance of convergence, the iterative procedure was not attempted. For objects that occurred in very large cutouts (for example, faint stars in the vicinity of a bright star), the centroider was also omitted, due to resource constraints.

\subsubsection{Classification Parameters}

In order to provide additional information for the image classifier (see Table 2), two additional types of numerical features known as spike parameters and texture parameters are computed. Bright point sources on Schmidt plates display diffraction spikes. These were used to good effect in GSC-I for star/nonstar discrimination in the brighter magnitude ranges. Spike parameters result from the comparison of the image gradient along the spike, which should be weak, with the gradient along the thin dimension of the spike, which should be strong. Texture parameters are based on the co-occurrence matrix of the object cutout (with fractional pixel allocation applied in the case of deblended objects), see Malagnini (1983) for further details.

\subsubsection{Summary of Known Limitations}

The sky processor is less efficient in very crowded fields or around bright stars and halos, such as filter ghosts. As already mentioned, the object detection procedure fails in the presence of large objects, such as bright stars or galaxies, or connected objects that will require a cutout greater than $4.25^{\prime} \times 4.25$ '. Also, the use of a unique sky standard deviation for the whole plate becomes problematic at some low galactic regions in the presence of high extinction gradients; here, local detection thresholds should be determined for optimal object detection.

GSC-II has both long- and short-exposure plates along the galactic plane. In these regions, the plate processing pipeline was often run with customized parameters.

Faint objects in the halos of bright ones were often missed due to both the sky problem described above and a failure of the deblender task. Objects brighter than 13th magnitude were sometimes broken up into smaller objects. The halo or spikes of a bright star were usually split up and identified 
as artifacts; if these artifacts were matched between successive plates or if they were classified as star/nonstar objects, then they were included in the export catalog. In some cases, faint objects close to a bright stars have been erroneously detected twice: firstly, as a secondary component in the wide cutout of the bright star, and secondly as a direct detection.

\subsection{Plate calibrations}

For each plate used in the GSC-II, the results of the image processing pipeline discussed in the previous sections provide a list of identified objects with position measurements (and errors), integrated density measurements, and a set of shaperelated parameters. These are the raw data used by the calibration procedures to derive celestial coordinates, natural magnitudes, and classification.

\subsubsection{Astrometry}

The astrometric calibrations of the GSC 2.3 are based on reference catalogs that provide local representations of a fundamental coordinate system. The chosen astrometric reference catalogs are the ACT and Tycho-2, as described in Section 2.2. Since, on average, there are a few hundred reference stars per plate, the plate positions are tied directly to the reference catalog and hence to the ICRS. The brighter stars appearing in the reference catalogs, i.e., $V<8.5$, are heavily overexposed on the Schmidt plates and thus not useful in calibrating the plates.

In order to compensate for differential refraction across the plate, the measured $x$ and $y$ pixel coordinates are pre-corrected for refraction using the method described by Konig (1962). Determining the relationship between $x, y$ and their associated equatorial coordinates was done by traditional global plate modeling, after an equidistant projection was applied to transform equatorial into tangential coordinates. We found that a quadratic model was suitable to represent the relationship between the $x$ and $y$ plate measures (precorrected for refraction) and the equidistant tangential coordinates $(\xi, \eta)$. Only the objects flagged as astrometric reference stars were used in a leastsquares reduction to determine the coefficients of the model. These coefficients were then used to map all the $x, y$ measurements to the associated $(\xi, \eta)$ values. Having started calibrating the plates in 1998, when Tycho-2 was still not available, the polynomial plate model was determined from the ACT for $\approx 73 \%$ of the plates and from Tycho-2 for the remaining.

Once a significant number of plates from the same survey had been reduced (approximately 100), the estimated tangential coordinates were compared to those calculated from the reference star's coordinates, and used to correct for residual systematics according to the precepts of Taff et al. (1990). Specifically, for each plate the reference stars' residuals were accumulated in $4.4 \mathrm{~mm} \times 4.4$ $\mathrm{mm}$ bins, then added and averaged over all the plates to build a plate-based astrometric mask, which yields the systematic pattern the global plate model failed to remove. A separate astrometric mask for each survey was determined using the ACT catalog. Figure 6 shows the mask applied to the northern blue survey plates (XJ).

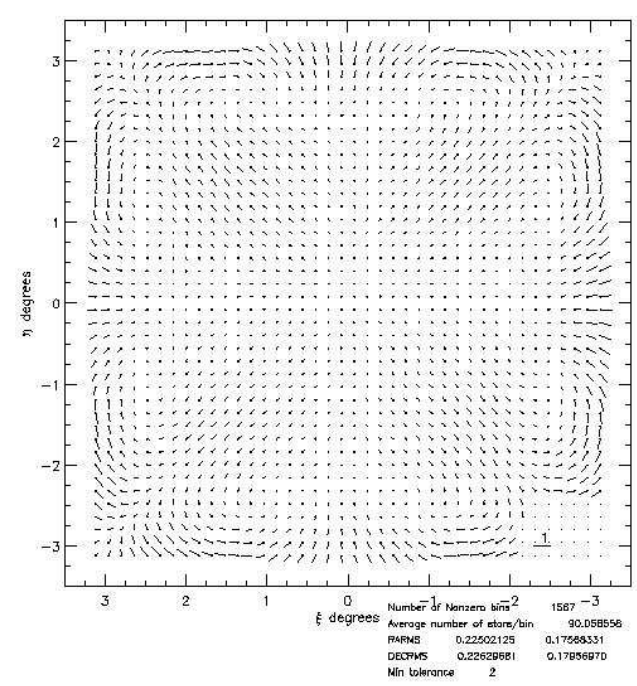

Fig. 6. - XJ astrometric mask $40 \times 40$ bins. The scale of 1 arcsec is plotted in the lower right-hand corner. 


\subsubsection{Photometry}

GSC-II magnitudes were derived via modeling of the nonlinear density-to-intensity response of plate-based photometric calibrators, in the natural system defined by each individual plate (Table 1). The calibrators are essentially based on JohnsonKron-Cousins B, V, R standards from the Guide Star Photometric Catalog (GSPC) I (Lasker et al. 1988) and II (Bucciarelli et al. 2001); additionally, $B_{T}, V_{T}$ photometry of Tycho stars was included in the calibration to constrain the bright range of the response curve.

Initially, the magnitudes of photometric calibrators have been transformed from the original Johnson-Kron-Cousins and Tycho photometric systems to the natural system of the plates by means of linear interpolation of the colorcolor point diagrams shown in Figure 7 These were generated by synthetic photometry (IRAF SYNPHOT package Bushouse and Simon 1994) of stellar spectra from the Bruzual-Persson-GunnStryker Spectrophotometry Atlas (Gunn and Stryker 1983). Note that in the usual case of photometric standards having both $\mathrm{B}-\mathrm{V}$ and $\mathrm{V}-\mathrm{R}$ available, the transformation based on the color closer to the requested photographic passband was utilized (e.g. $B_{J}-V_{\mathrm{pg}}$ and $V_{\mathrm{pg}}-R_{F}$ as a function of $B-V$ and $V-R$, respectively).

The instrumental photometric parameter of choice was the integrated density, defined as the logarithm of the total density,

$$
P=\log _{10} \sum_{i, j}\left(D_{i, j}-D_{\mathrm{bck}}\right)
$$

calculated as the sum of the PDS-measured density, $D_{i, j}$ (ADC units), above the local background, $D_{\text {bck }}$, for each pixel pertaining to the selected object. The fit to standard magnitudes was performed using Chebyshev polynomials, namely:

$$
m=\sum_{i=0}^{4} A_{k} T_{k}\left(P^{\prime}\right)
$$

where $T_{0}(x)=0.5, T_{1}(x)=x, T_{2}(x)=2 x^{2}-1$, $T_{3}(x)=4 x^{3}-3 x$, and $T_{4}(x)=8 x^{4}-8 x^{2}+1$, $A_{k}$ are the unknowns to be estimated, and $P^{\prime}$ is the photometric parameter linearly scaled to map the range $[-1,1]$, where Chebyshev polynomials can be suitably applied. A typical pipeline output, as given in Figure 8, shows that these polynomial bases fit nicely the characteristic curve over a large range of densities, in particular at the faint end, where the large majority of the objects lie. In this respect, extensive experiments have demonstrated that the use of polynomials of order higher than fourth was not advisable because of their high instability toward the plate magnitude limit. The resulting coefficients were then used to convert the measured integrated densities into magnitudes for all the objects detected on the plate.

As a protection against gross errors in the calibration, reference stars with large residuals were rejected from the fit, according to the criterion $\Delta$ $>\sigma \times \epsilon_{1}$, where $\Delta$ is the residual of the star under test, $\sigma$ is the formal error of the fit, and $\epsilon_{1}$ is an adjustable parameter, generally set equal to 2 . The procedure was stopped when no further stars were rejected, or when the difference of formal error between successive iterations was less than $\epsilon_{2}$, an adjustable parameter typically set at 0.03 . Occasionally, such iterations failed to achieve formally acceptable solutions. In this case, manual intervention was required, for example, to change the order of the Chebyshev polynomial or to modify the acceptance criteria for the reference stars.

The color transformations become poorly defined at extreme color indices; therefore, only standard stars in the ranges of $-0.34 \leq B-V \leq 1.85$ and $-0.14 \leq V-R \leq 1.42$ were accepted. Moreover, those stars for which the integrated density could not be adequately determined were discarded from the initial set. This could depend on a variety of reasons, such as image processing failures, defective images, and centroiding biased by faint components of unresolved blends. Therefore, in general, stars brighter than 8.5 mag as well as deblended objects were eliminated. Since Tycho stars are generally more numerous than GSPC-II stars on any plate, thereby overconstraining the bright side of the fit, a weighting scheme determined by the magnitude range of the selected catalog divided by its number of stars was applied. Figure 9 shows an example of the spatial distribution of the photometric calibrators, with the GSPC-II sequence near the plate center and the Tycho stars located all over the plate.

Generally, GSPC (I/II) stars range between 9 th to 19 th magnitude (in $V$ ), while the GSC objects are both brighter and fainter, such that 

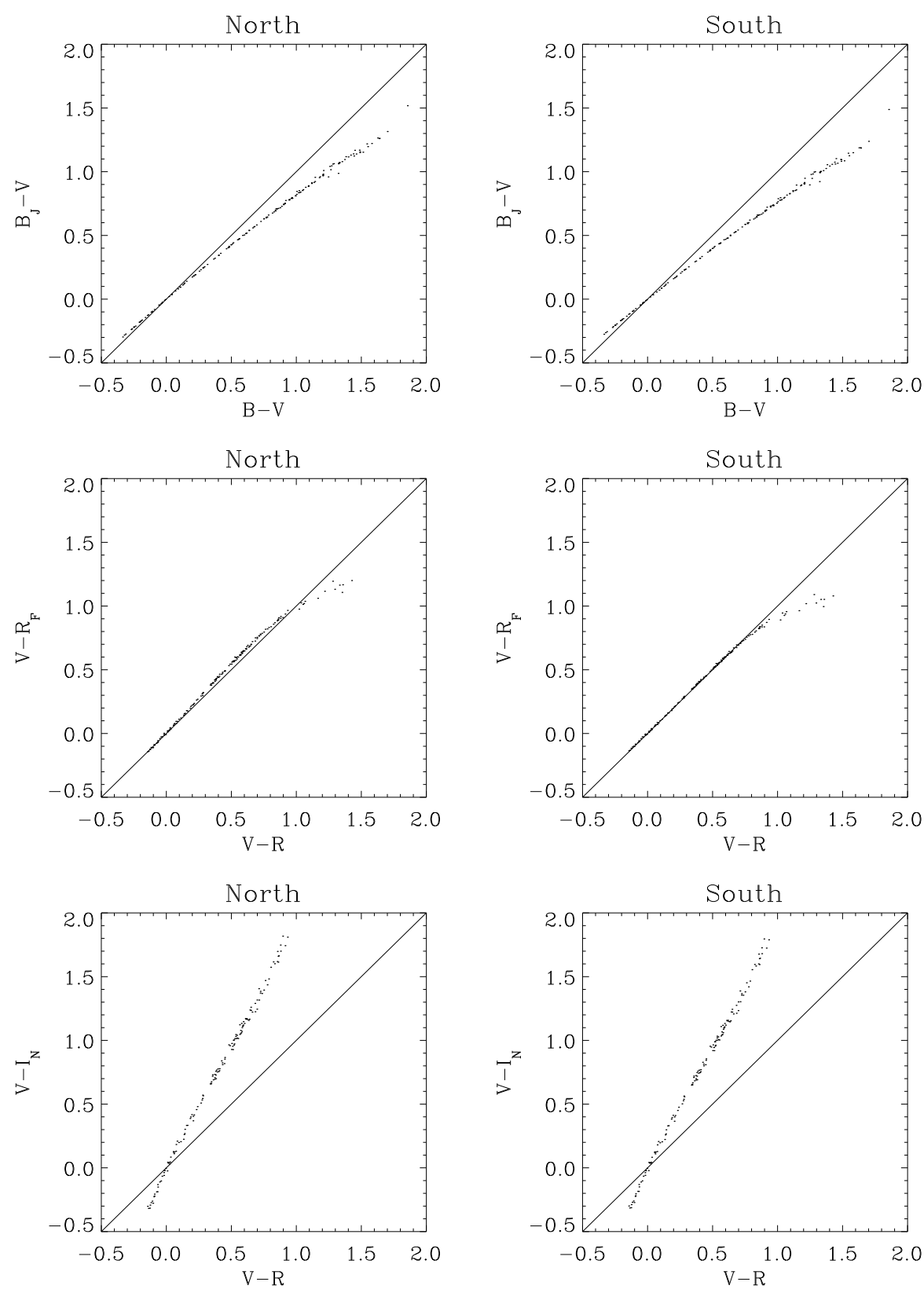

Fig. 7.- Color-color plots used to transform GSPC-I/II calibrators from the $\mathrm{BVR}_{\mathrm{c}}$ photometric system to photographic passbands $B_{J}, R_{F}$, and $I_{N}$ ( $x=y$ lines are displayed for reference). 


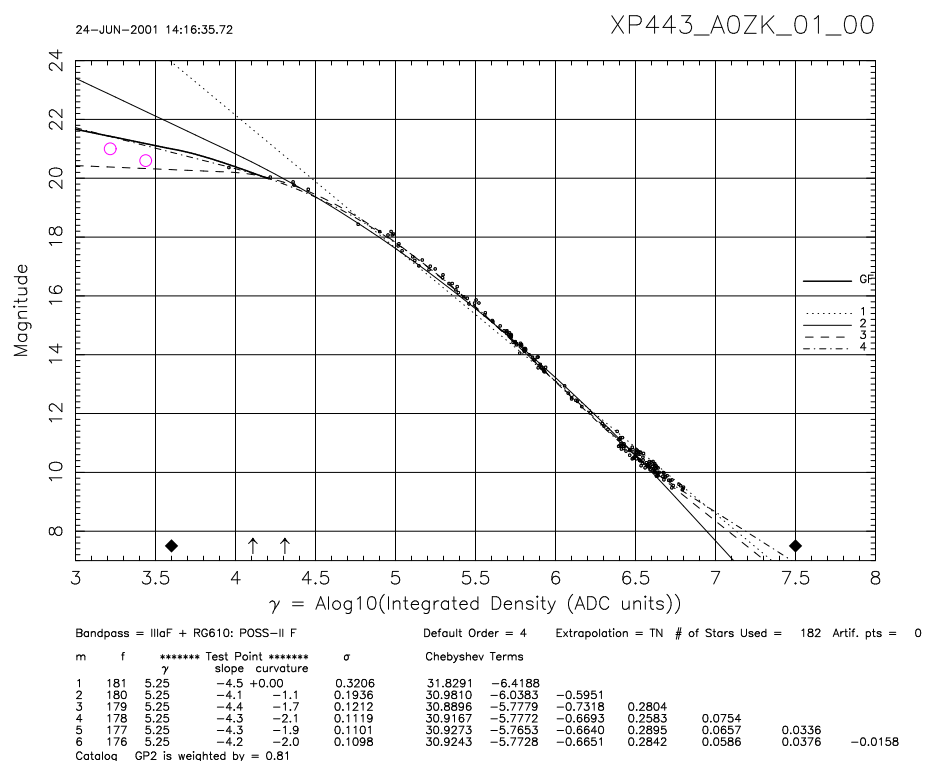

Fig. 8.- Photometric calibration of plate XP443. Small circles represent GSPC-I/II and Tycho stars used to fit a fourth-order polynomial (dot-dashed line) on $R_{F}$ vs. $P$. Artificial stars (large circles) were not applied in this case.
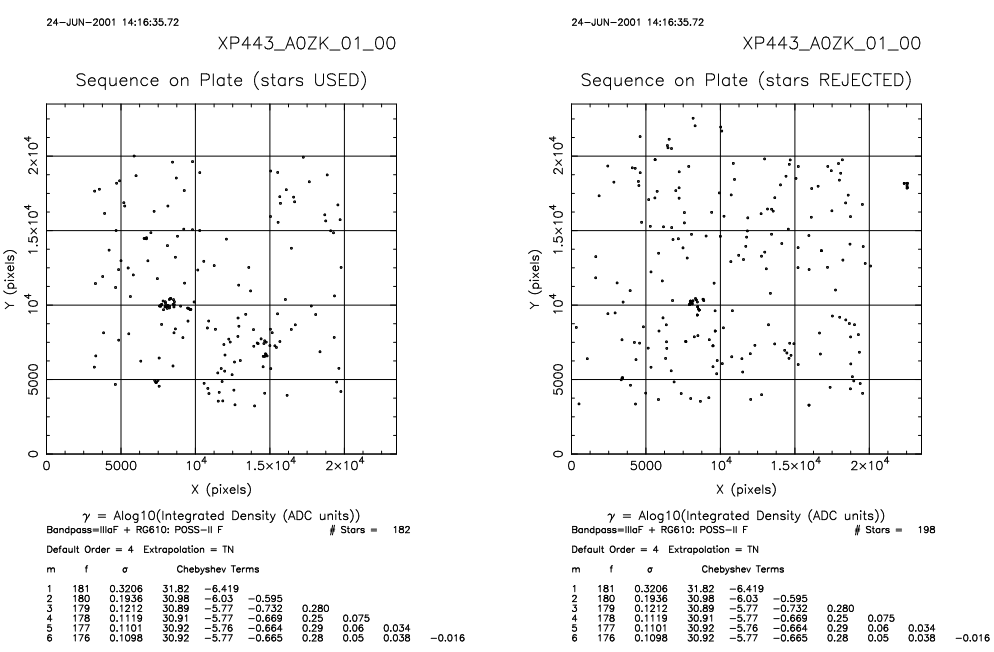

Fig. 9.- Photometric calibration of plate XP443. Plate distribution of the photometric calibrators (left panel). Note the faint GSPC-I/II sequence located at $X \approx 8000$ and $Y \approx 10,000$, while Tycho stars are spread over the plate. The right panel shows the objects rejected for various reasons (blends, outliers, colors outside the range, etc.), plus a GSPC-I/II sequence very close to the plate border $(X \approx 22,000, Y \approx 18,000)$. 


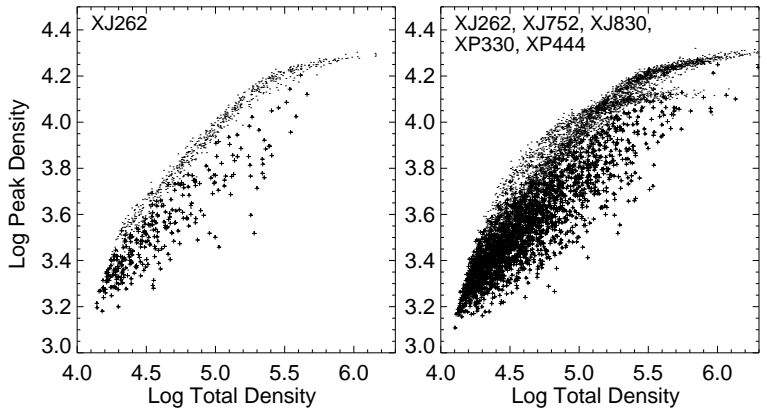

Fig. 10.- Total density vs. peak density for stars (dots) and galaxies (plusses) measured on GSCII photographic plates. (a) The distribution for a single plate (XJ262) is well defined and could be used for classification. (b) The distribution for five plates differs for different plates and so a classifier for all plates could not be constructed from these parameters.

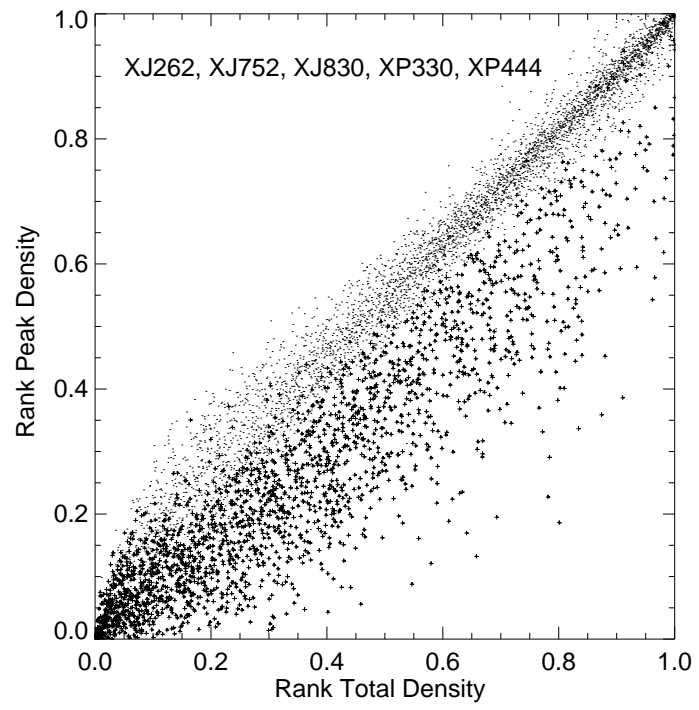

Fig. 11.- Distribution of total density vs. peak density for objects from Figure 10 after transformation using ranks. Stars and galaxies from different photographic plates separate very well. An accurate classifier can be constructed based on these ranked parameters. extrapolations from the photometric reductions are required. Because the images of bright stars (brighter than 9.0) are heavily exposed on Schmidt plates, their integrated density is not well determined. Therefore, no extrapolation of the photometric reduction for objects brighter than those used in the calibration was performed, and the corresponding magnitude flag set to -99 . In the latest catalog version, all the bright magnitude stars having a Tycho-2 counterpart were replaced with the Tycho-2 magnitude value (see Section 4).

The method of extrapolation used for the faint stars was tangential, i.e., a linear extrapolation with the slopes set to be tangent to the end points (brightest and faintest standard stars) of the fitted function. However, by using a fourth-order Chebyshev polynomial, the extrapolated region was often unstable. In order to stabilize this region, we introduced an artificial reference star and assigned to it the plate magnitude limit of the surveyobtained from the literature and reported in Table1-which was associated to the integrated density equal to that of the smallest detectable image on the plate. In general, the use of an artificial point improved the situation, especially on plates where the faintest GSPC stars were in the range of 16-17 mag. On the other hand, in cases where the standard sequence had a fainter limit, the use of the artificial star was not needed and could even cause GSPC stars to be rejected by the iterative process - an undesirable effect - so the artificial point was not used on these plates.

Variations in the photometric sensitivity across the Schmidt plates are caused by sensitivity variations in the emulsion, telescope vignetting, and point-spread function (PSF) changes across the field. We have not corrected for such effects with this release.

It should also be emphasized that the photometric pipeline, which is tuned for point-like objects, systematically overestimates the magnitude of bright galaxies $\left(R_{F}<18\right)$ which do not have a stellar PSF. This limitation was acceptable since the primary purpose of GSC-II is to provide guide stars for telescope operations. It is possible that a future version may use a different calibration technique to provide more accurate galaxy magnitudes. 


\subsubsection{Image Classification}

The main motivation for classification in the GSC series is to reliably identify stars, which can be used for guiding, from "nonstars", which cannot. At the scale of the Schmidt plates, such "nonstars" include not only galaxies, but blends of overlapping images which were not resolved into individual objects by the deblending step of the plate processing pipeline. A third category, "artifact", is used to label images that appear on the plates but do not correspond to astronomical objects - for example, pieces of diffraction spikes, halos, etc. In a subsequent release of the catalog, we plan to replace the generic "nonstar" category by "galaxy" and "blend" labels, at least for the brighter objects where such discrimination is possible. In general, nonstars can be considered to be primarily galaxies far from the galactic plane, and primarily blends close to the plane.

A set of 30 features, describing the shape, uniformity, and brightness, was calculated for each object, as detailed in Table 2, This set was selected based on good star/galaxy separation in feature plots. The "raw" features were then transformed by the statistical process of "ranking", that is, ordering a population of objects by the value of the feature, and mapping the full range of the feature into a range of 0:1. The ranked features were then used in the remainder of the classification process (see White 1997). This ranking has the effect of significantly reducing plate-to-plate variations in the features (see figures 10 and 11), removing the necessity of creating a specific training set for each plate. This can be illustrated by considering the case of ellipticity, a common classification feature: the roundest objects on any plate are likely to be stars, even if one plate is poorly guided compared to the other. The ranking was performed separately in different zones of the plate, which was divided typically into a $7 \times 7$ grid, whereas some especially crowded plates were gridded more finely due to memory limitations. Objects lying near the grid boundaries were interpolated.

An oblique decision tree (OC1 Murthy et al. 1994) was used to perform the classifications. Unlike classical, or axis-parallel, decision trees that can split on only one variable at a time, OC1 can perform splits on a linear combination of all the features. This permits OC1 to find and sensibly exploit relationships among the image features. To lower the variance contribution to the classification error, a set of five decision trees were created from the training set of 5334 hand-classified objects based on stars and galaxies from the deep CCD catalog of Postman et al. (1996), using different randomizations in the tree-building process to produce five pruned trees from the same data. During the plate classification task, each object was classified by all five trees, and the results were voted to produce a final, single plate, classification.

The training set and decision trees were tuned to produce the fewest number of misclassifications over the entire population of objects on the plate. Since the majority of the detected objects are faint objects, and since bright and faint objects classify differently, this effectively meant optimizing for faint objects at the expense of bright objects.

\section{The GSC-II Database}

The COMPASS database is the primary repository for all the data from the plate image processing pipeline. The GSC-II is exported from the database as a subset of the multiband and multiepoch surveys because the same objects on the sky appear on different plates. The database establishes the associations between objects on the different surveys and provides a central indexing scheme for which the export task can extract the calibrated parameters for each object.

\subsection{Database Structure and Operations}

The database architecture is built on a hierarchical object-oriented framework using Objectivity, a commercial Object-Oriented Database Management System. We regarded as important elements for the selection of this system its low development costs and its scalability to multi-terabyte data sets, along with the capability of mapping the object modeling of the raw data directly into the database schema. The latter has allowed us to identify a unique correspondence between the plate-based raw data and partitions of the celestial sphere (sky regions) through the creation of a federated database file system (see diagram in Figure 12), with each plate covering multiple regions and each region containing several overlapping plate 


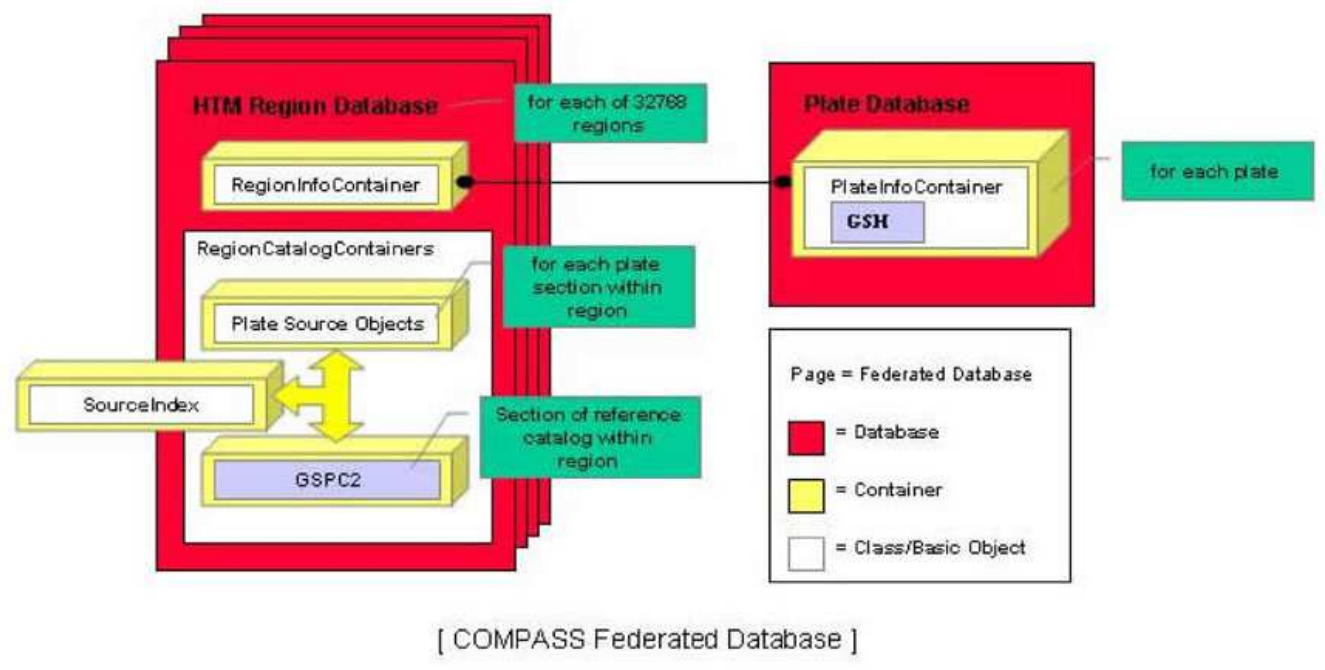

Fig. 12.- Representation of the GSC-II database structure. 
sections.

The subdivision of the celestial sphere is based on the HTM (Hierarchical Triangulated Mesh Kunszt et al. 2001), a spherical quad-tree index library developed by the Sloan Digital Sky Survey (SDSS) project at Johns Hopkins University. The regions are approximately equal area of the order of 1 square degree. Several large-scale archives have adopted HTM to facilitate interoperability for data correlations in addition to providing a high-speed spatial index into a data set.

Each of the HTM regions in the database contains a number of "containers" corresponding to the plate measurements, the reference catalog data (GSC-I, GSPC-II, Tycho-2, ACT and SKY2000), and a master index which identifies each unique object on the sky within that region and has links to the individual observations and catalog entries.

\subsection{Object Matching and Naming}

As each plate was processed and loaded into the database, a matching task was run which identified objects on that plate with previously loaded objects. Object matching was based on position only and was run iteratively with search tolerances increasing from 1 to 4 arcsec. The matching code computed the distance with respect to all the previous observations belonging to any source within the searching radius and assigned the new object to the source containing the nearest neighbor. This procedure is repeated with a larger radii on the remaining objects and unmatched objects are added as new sources to the master index.

A known problem with the matching is due to noise fluctuations in the extended saturated cores of very bright stars resulting in the detection of spurious objects. Typically, these bright stars are contained in the SKY2000 and Tycho-2 catalogs, so the matching of these spurious objects to the same catalog entries is the cause of multiple entries in the GSC-II. This is not a problem for HST operations since the Tycho-2/SKY2000 values take precedence but we plan on fixing this issue in a later release.

In order to maintain backward compatibility for $H S T$ operations, the master index was preloaded with the GSC-I objects so that the GSC-I identifications were maintained. As objects were added to the index, an internal identification was as- signed which was the HTM container name (e.g., N012301) plus a running sequential number. This was adopted to aid fast object lookups within the COMPASS database. It should be noted that this name is converted to a 10 -character base-36 number known as the HSTid in GSC2.3 and later and that this is the "official" name. This was done because of the ten-character limit for the Guide Star names throughout the HST ground systems and flight software once $H S T$ pointing switched over to using the new catalog for pointing.

\subsection{The Export Catalog}

The GSC 2.3 export catalog provides positions, multicolor photometry, and morphological classification for about one billion unique objects. For each source, the GSC-II database may contain multiple measurements deriving from the observations matched from the overlapping multi-epoch and multicolor plates. In this section, the selection criteria adopted to filter the available data are illustrated. It is important to recall in this context that the main driver for the GSC-II project was to support and/or to improve on the efficiency of observation planning and operations in space and ground observatories which sponsored the project; the catalog extraction rules ultimately define the properties of the catalog released.

The magnitude limits of the export catalog were set at $B_{J}<19.5$ or $R_{F}<18.5$ for the first public version, GSC 2.2, released on 2001 July, while for the GSC 2.3 almost all the objects down to the magnitude limits of the plates (see Table 1) have been extracted from the database and included in the export catalog.

The magnitude limits of the GSC 2.2 basically correspond to the range which satisfies our guide star requirements for telescope operations in terms of positional and photometric accuracy. In particular, the colors provided in the export catalog are indispensable for both bright-object protection of the MAMA detectors on HST and adaptive optics operations of ground-based telescopes. The classification between stellar sources and nonstellar objects (extended objects or blends), is critical for the selection of both guide stars and adaptive optics reference stars and the GSC-II is sufficiently reliable within the stated magnitude range.

The deeper GSC 2.3 release was mainly mo- 
tivated by our commitment to distribute to the international astronomical community a large and valuable data set for a wide variety of astrophysical studies. Moreover, GSC-II data have been already used for various technical analysis which required a complete deep all-sky catalog, such as the guide star studies for the JWST (Spagna 2001; Stys and Kriss 2003), and the definition studies of the Gaia mission (Drimmel et al. 2005).

\subsubsection{Source selection.}

All the database entries were screened, but only objects detected and measured at least on one IIIaF or IIIaJ or IV-N plate were exported. Objects classed as defect on only one plate were discarded. A magnitude threshold, $m \leq 25$, significantly fainter than the plate limits of all the photographic surveys was applied in order to prune both faint false positive detections and spurious objects.

In order to improve the completeness and accuracy of the bright guide stars, which are strongly saturated in the GSC-II long-exposure plates, the GSC 2.3 has been supplemented with data from the Tycho-2 and SKY2000 (Mvers et al.2002) catalogs which contain stars down to $V \approx 12$ and 8 , respectively. The data for any given object were taken preferentially from the Tycho-2 when available, then SKY2000 and finally from the Schmidt plate measurements.

The GSC 2.3 also contains the names of the GSC-I objects that have been matched with the GSC-II objects.

\subsubsection{Astrometry.}

Generally, GSC 2.3 provides positions, $(\alpha, \delta)$, measured on the red IIIaF plate, and multiple measurements are resolved by selecting the closest solution to the plate center. In case the object was not detected on any IIIaF plate, then the positions from the blue IIIaJ, visual IIaD, and infrared (IR) IV-N plates have been used following this priority order. The position epoch is set accordingly. The position errors are estimated by means of a simple general model aimed to provide conservative uncertainties for telescope operations. These figures do not correspond to formal errors and cannot be used to define confidence limits in a statistical sense.
If a source was selected from the Tycho-2 catalog, the GSC 2.3 provides the positions, the proper motions and the mean observing epoch in right ascension (R.A.), which is slightly different from the declination (decl.) epoch in the Tycho2 catalog. For SKY2000 objects, coordinates and proper motions are provided at epoch J2000.0. It should be noted that at this time we are not publishing computed motions due to a small systematic error in the coordinates (see Section 5.3)

\subsubsection{Photometry.}

Photographic magnitudes $B_{J}, R_{F}, V_{\mathrm{pg}}, I_{N}$,plus the blue POSS-I $O$ are provided, if available, for all the exported objects. In case of multiple measurements, which may occur at the plate borders where different plates of the same band overlap, we selected the observation closest to the plate center, without applying any averaging procedure. As a result of the main source selection criterion, at least one $B_{J}, R_{F}$, or $I_{N}$ magnitude is always present, while additional blue $O$ and $V_{\text {pg }}$ magnitudes are given for the objects also matched on the POSS-I O, Palomar Quick-V, and SERC Quick-V surveys. As for the positions, magnitude errors are approximate and conservative estimates.

In the case of Tycho-2 objects $B_{T}$ and $V_{T}$ magnitudes are provided, whilst SKY2000 objects may provide additional Johnson UBV magnitudes.

\subsubsection{Source Classification.}

Classification voting was implemented on the set of observations for an individual sky source. For each source we have at least two classifications coming from the plates in the different bands, but we may have up to 25 if the object falls into a region of multiple plate overlap. The final published classification of a matched object is the mode of the individual classifications (ignoring artifact classification on the assumption that any object matched on two plates is real). Unmatched artifacts are excluded from the catalog. In the event of a tie, e.g. as many stellar as nonstellar votes, we decided in favor of non-stellar based on our experience that it is easier to misclassify a galaxy as a star rather than vice versa. Individual classifications based on older $25 \mu \mathrm{m}$ data are excluded from the voting to ensure consistency. 
Each source was classed as star or nonstar and, in the case of ties, nonstar classifications were selected to ensure highest quality stellar sources. We used only the classification of the objects derived from the higher-resolution scans (1.0" pixel); classification of low resolutions scans $\left(1.7^{\prime \prime}\right.$ pixel) were utilized in the rare case of objects without any detection on the high-resolution scans. A quality flag which indicates an unanimity voting was also added to the export catalog. "Defects" revealed and matched on more than one plate were classified as nonstars.

Since this catalog is primarily for the selection of guide stars and the GSC-I classification was strongly biased toward this, then if an object was cross-matched with the GSC-I that classification was adopted and it superseded the voting scheme described here.

Finally, the morphological parameters (ellipticity and orientation angle) of the image used to derive the positions were exported too.

\subsubsection{GSC 2.3 Export file format}

The export catalog is a distributed FITS file system based on the linear quad-tree HTM sky partitioning scheme, with a level-6 triangulation implementation (see Kunszt et al. 2000). Each FITS file includes all calibrated celestial sources for a single triangular section of sky (HTM level 6 leafnode), roughly corresponding to one square degree of sky data. The file names are the quadtree representation of each triangular section, with the first character being either an "N" or "S" representing north or south.

Each FITS file contains three sections, the required primary header, an ASCII table extension (containing a lookup table that access software can use to speed up reading data subsets), and a BINARY table containing the actual catalog data.

Table 3 shows the fields officially released with GSC 2.3, including object names, astrometric and photometric parameters, object classification, as well as some auxiliary parameters and flags. The binary FITS tables also include several empty fields for future releases or containing data for internal testing; these are not part of GSC 2.3 and must not be used nor distributed.

As explained in the notes of Table 3, all the photometric fields may contain magnitudes for dif- ferent, although similar, passbands. GSC-II users can identify the exact filter by looking at the magnitude-code fields, for which we list in Table 4 the photometric codes used in GSC 2.3.

Further details on the processing of the GSC-II objects are given in the source status flag, whose structure is described in detail in Table 5. Tycho2 stars are identified by a Quality Status Flag of $999999 n n^{1}$ and SKY2000 stars are identified by a Quality Status Flag of 88888800.

\subsection{GSC 2.3 distribution}

The export catalog, GSC 2.3, requires a storage capability of approximately $170 \mathrm{~GB}$ and it is currently maintained by STScI and INAFOATo. Copies of the catalog have been already distributed to the project patrons (ESO, GEMINI, ESA) as well as the main astronomical data centers and several research institutions. Due to limited resources, the GSC-II team does not plan to provide an extensive distribution service of the complete catalog and requests will be reviewed on a case-by-case basis. However, public access to GSC-II is available through both Web and VO-compliant (Virtual Observatory) interfaces at STScI, OATo and CDS.

\section{Catalog Properties}

The application of the "selection" criteria explained in the previous section generated the export catalog, GSC 2.3, containing 945,592,683 objects in total, classified as stars $(22 \%)$ and nonstars $(78 \%)$.

Table 6 presents the global statistics of GSC 2.3, including the number of objects for which photographic magnitudes, $B_{J}, V_{\mathrm{pg}}, R_{F}, I_{N}$, are provided, and the number of objects related to GSC-I, Tycho-2, and SKY2000.

All-sky cumulative starcounts in the $B_{J}, R_{F}$, $I_{N}$ are provided in Table 7.

The GSC2.3 all-sky density map in galactic coordinates is presented in Figure 13, which includes both stellar and nonstellar objects. The counts are dominated by stars close to the galactic plane, and most of the objects classified as nonstars are actually unresolved stellar blends belonging to crowded

\footnotetext{
${ }^{1}$ Here $n n$ is the number of observations used to compute Tycho 2 position and proper motion.
} 
fields toward low galactic latitudes. Also, an analysis of the counts shows that at $R_{F} \leq 20$ about $4 \%$ of the GSC2.3 sky has a density of above $10^{5}$ objects per square degree, reaching in some cases up to $10^{6}$ before getting confusion limited.

The reliability of the astrometry, photometry, and classification of GSC 2.3 can be investigated by comparisons with independent reference material as well as by internal checks. The latter take advantage of the significant plate overlap and can probe the degree of catalog consistency down to the very plate limit; nonetheless, they are not discussed here because, since the plate-based nature of GSC-II is not preserved in the way the export catalog was constructed, an error analysis based on the plate overlaps would not give the right information about the GSC 2.3 errors.

\subsection{Comparison Catalogs}

The UCAC 2 (Zacharias et al. 2004) contains almost 50 million objects down to $R \approx 16$, covering the entire southern hemisphere and a large fraction of the northern one (complete to $\delta=40^{\circ}$, and up to $\delta=52^{\circ}$ in some areas). Its astrometric precision is between 40 and 70 mas per coordinate depending on magnitude, and systematic errors are within 10 mas; these values are lower than the typical GSC-II errors, except for the faintest UCAC 2 objects, so that this catalog appears ideal to reveal the astrometric precision of GSC 2.3. The only drawback is the magnitude limit being significantly brighter than that of GSC 2.3. It is worth noting that the next release of this catalog (UCAC 3) which is all-sky would be an excellent reference catalog to recalibrate the plates since it is deeper than Tycho-2 and would reduce some of the systematic errors in GSC-II that we describe later.

In order to extend the astrometric error analysis to fainter magnitudes we also matched GSC 2.3 with SDSS DR5 (Adelman-McCarthy et al. 2007). This provides astrometrically and photometrically calibrated data for an area of $\sim 8000 \mathrm{deg}^{2}$ around the northern Galactic cap; a total of about $6 \cdot 10^{6}$ objects including stars, galaxies and quasars are in common with GSC 2.3. For point sources brighter than $r=20$, the astrometric accuracy of SDSS is 75 mas per coordinate with an additional 20-30 mas systematic error (see Pier et al.2003). At the survey limit $(r=22)$, the astrometric accuracy is limited by photon statistics to approximately 100 mas root mean square ( $\mathrm{rms}$ ). The photometric calibration is accurate to roughly $0.02 \mathrm{mag}$ in the $g$-, $r$-, and $i$-bands (Ivezić et al. 2004), so the SDSS can also be used to estimate the GSC 2.3 photometric errors. A comparison against GSPC-II also provides a final global validation of the photometry.

In order to compare the astrometry to an all-sky catalog of similar size, yet derived from independent data, we also matched GSC 2.3 against the Two Micron All Sky Survey (2MASS; Skrutskie et al. (2006)) Point Source Catalog (PSC). This lists 470,992,970 objects distributed over the entire sky, providing near-IR photometry with signal-to-noise ratio $(\mathrm{S} / \mathrm{N})$ better that 10 down to $J \simeq 14.2, H \simeq 15.1$, and $K_{s} \simeq 14.2$. Note that the GSC 2.3 does include the bulk of 2MASS objects, as these basically span the entire magnitude range of GSC-II, thanks to the wide color spectrum covered by both catalogs and the natural spread of stellar temperatures and galactic extinction. The astrometric accuracy of 2MASS is 70-80 mas per coordinate over the magnitude range $9<K_{s} \lesssim 14$, while for brighter sources it is approximately 120 mas. For fainter sources the error increases monotonically. The systematic errors are of the order of 10 mas, on average, and up to a maximum of 25 mas for the worse cases.

The USNO-B (Monet et al. 2003) or the Southern SuperCosmos Survey (SSS, Hambly et al. 2001) were not used as external checks since they are derived from much of the same plate material as GSC-II, so do not provide independent measurements of the quality. As a sanity check GSC 2.3 was compared to both USNO-B and SSS but the differences did not reveal anything new that is considered significant.

In addition to the large-scale comparisons performed with the above catalogs, we further checked object classification against the selectedby-eye galaxy catalog of the NPM/SPM programs (Klemola et al. 1994; Platais et al. 2002) and various NGP surveys (Infante 1993; Graham et al. 2007), the APM (Irwin and McMahon 1992; Loveday 1996) and APS (Cornuelle et al. 1997) automated galaxy classification catalogs. 


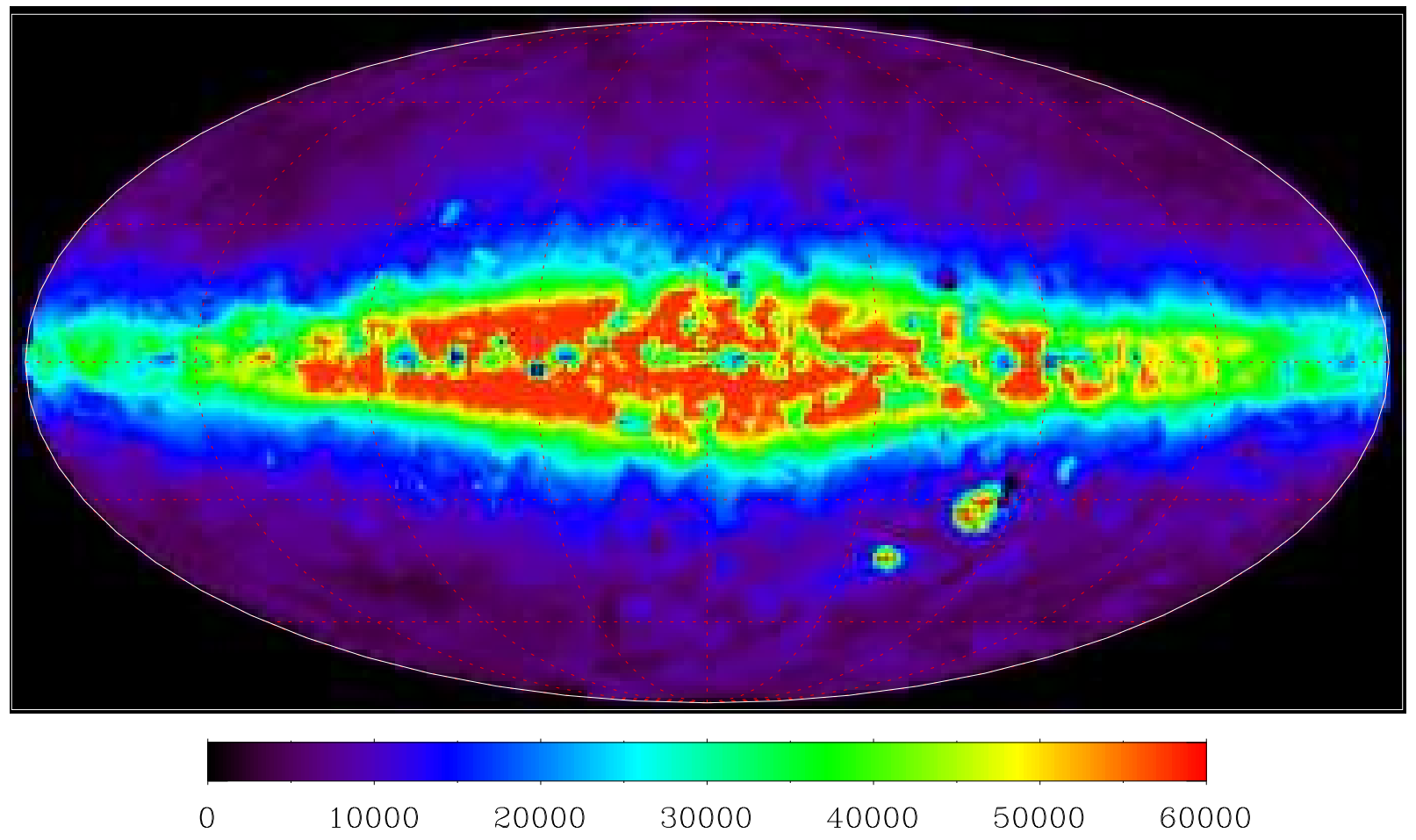

Fig. 13.- GSC 2.3 all-sky map. Cumulative counts in galactic coordinates, including both stellar and extended objects. The color scale indicates the GSC 2.3 density ranging from 0 to 60,000 objects per square degree. The image resolution is a smooth version of the densities obtained from the HTM counts. 

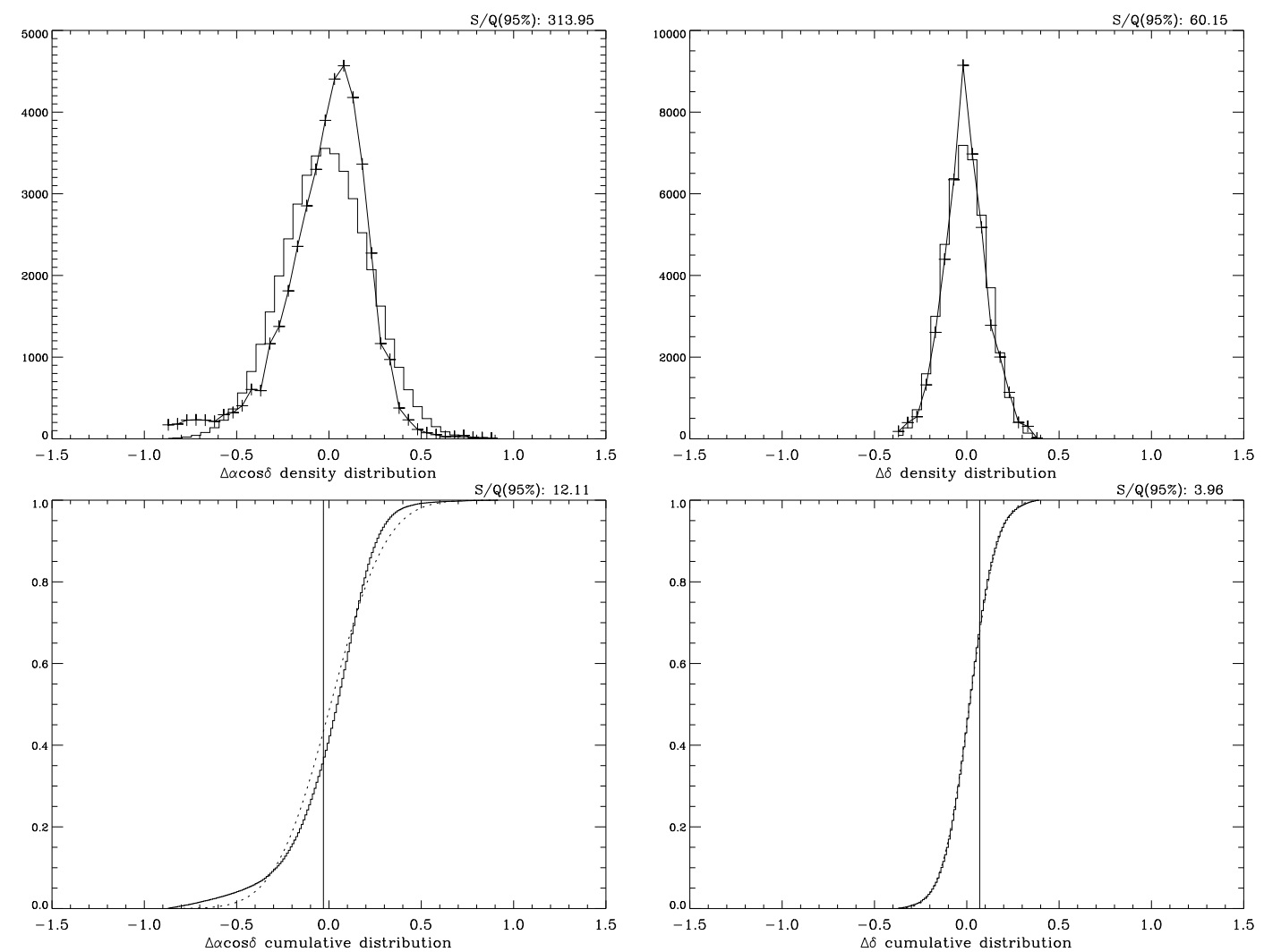

Fig. 14. - Comparison to the UCAC 2 catalog for a sky region centered at $0 \mathrm{~h}$, spanning all declinations, and in the magnitude range 14.5-15.0. The panels show the results of the $\chi^{2}$ (top panels, the histogram is the theoretical $\chi^{2}$ distribution), and K-S test (bottom panels, the dotted line is the theoretical distribution). The empirical distributions do not satisfy the tests $(S / Q>1$, see the text). This is attributed to the presence of residual systematic errors.

\subsection{Astrometric errors}

As discussed in Sect. 3.3 (astrometric calibrations), the GSC-II is affected by systematic errors, which are function of both position and magnitude. In the following, we investigate size and variation of such errors by comparing the GSC2.3 astrometry to the positions of selected external catalogs. These were calibrated with Tycho-2 stars, thus we are in a position to check at the same time on the degree of global and local adherence of the GSC 2.3 positional system to that of the ICRF reference frame. We also give an estimation of the positional random error for both point-like and extended objects, which is the relevant quantity in any application requiring knowledge of the relative astrometric precision.

\subsubsection{Statistical tests}

A purely statistical approach to test the degree of randomness of the astrometric errors is the method of hypothesis testing. Specifically, we applied on our data two goodness-of-fit tests, i.e., the $\chi^{2}$ and the Kolmogorov-Smirnov (K-S) tests, as detailed in the following.

Let $\epsilon_{i 1}$ and $\epsilon_{i 2}$ be the actual coordinate errors of star $i$ from the GSC 2.3 and the comparison catalogs, respectively. Assuming that $\epsilon_{i 1}$ and $\epsilon_{i 2}$ are independent normally distributed random variables with respective variance $\sigma_{1}^{2}$ and $\sigma_{2}^{2}$, then their difference $d_{i}=\epsilon_{i 1}-\epsilon_{i 2}$ is also normally distributed, with variance equal to $\sigma_{1}^{2}+\sigma_{2}^{2}$.

This assumption on the distribution function of $d_{i}$ was the working hypothesis to which the $\chi^{2}$ and the Kolmogorov-Smirnov tests were applied. Since 

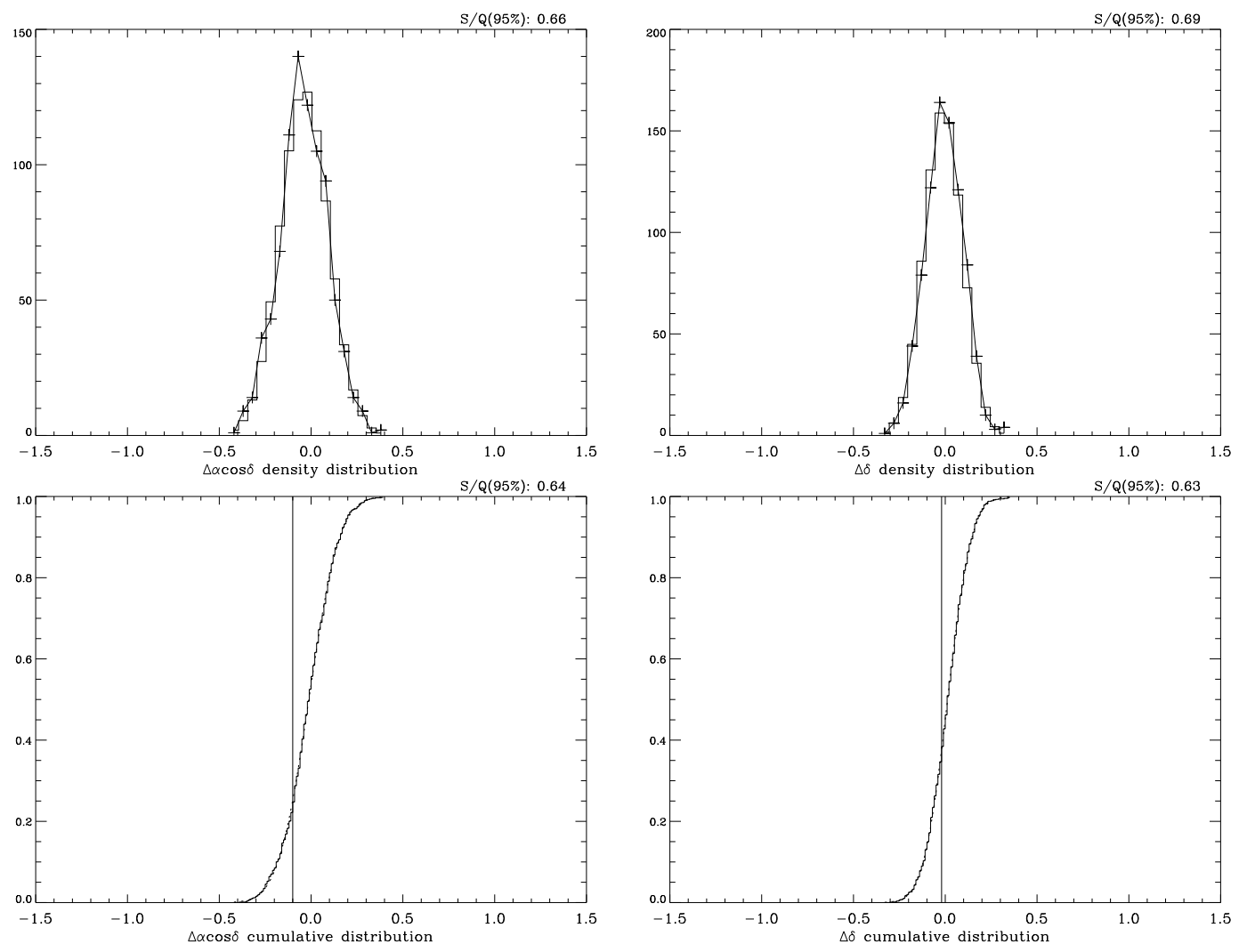

Fig. 15.- As in Figure14 these panels show the results of the $\chi^{2}$ and K-S tests for the GSC 2.3 versus UCAC 2 residuals, this time restricted to one plate and to the magnitude range $R_{F}=14.5-15$. In this case, both significance tests are satisfied $(S / Q<1)$.

each star has only one observation per plate, we had to resort to an "idealized" experiment in order to have the required redundancy. To this end, we considered measurements of different stars close in magnitude as belonging to the same parent population, i.e., each measure was treated as a single realization of the statistic under test. Such approach is correct as long as the measurement/model errors are independent (or marginally dependent) on sky/plate position or other effects not taken into account by the plate model; if any of these conditions is not satisfied, the goodness-of-fit tests are doomed to fail, whereas a successful test would confirm the existence of such conditions.

Assuming a Gaussian probability density distribution (pdf) of the $d_{i}$, we estimated first the population standard deviation by using the formula $\sigma=\sqrt{\frac{\pi}{2}} d$, where $d=\frac{1}{N} \sum_{i}\left|d_{i}-\langle d\rangle\right|$ is the sample mean deviation, which is less sensitive to outliers. Then, we applied a $3 \sigma$ rejection criteria to the data and calculated the usual sample mean $\mu$ and standard deviation $\sigma$ of the cleaned sample. Finally, by binning the residuals at $0 . " 05$ steps, we built the test statistic

$$
\chi^{2}=\sum_{i=1}^{k} \frac{\left(N_{i}-N p_{0 i}\right)^{2}}{N p_{0 i}}
$$

where $N$ is the total number of residuals, $N_{i}$ is the number of residuals in the i-th bin, and

$$
p_{0 i}=1 /(\sqrt{2 \pi} \sigma) \int_{x_{i}-\Delta x / 2}^{x_{i}+\Delta x / 2} \exp \left(\frac{-(t-\mu)^{2}}{2 \sigma^{2}}\right) d t
$$

the probability of a residual falling into the $i$ th of the $k$ bins, which can be evaluated using the error function. Note that the $\sigma$ and $\mu$ parameters used in the integral above are estimated from the sample itself. 
The approximate distribution of $\chi^{2}$, valid for large samples, is that of the chi-square probability function with $k-3$ degrees of freedom.

An alternative significance test we have applied to our sample is the double-sided K-S statistic, which is exact in the sense that does not require data binning.

The K-S test statistic is defined as

$$
D_{k}=\max \left|S_{k}(x)-F(x)\right|
$$

with

$$
S_{k}(x)= \begin{cases}0 & \text { for } x<d_{1} \\ k / N & \text { for } d_{k} \leq x<d_{k+1} \\ 1 & \text { for } d_{k} \leq x\end{cases}
$$

and

$$
\begin{gathered}
F(x)=1 /(\sqrt{2 \pi} \sigma) \int_{-\infty}^{x} \exp \left(\frac{-(s-\mu)^{2}}{2 \sigma^{2}}\right) d s \\
=0.5+\frac{\operatorname{erf}(x)}{2}
\end{gathered}
$$

where erf is the error function.

Both tests adopted a $5 \%$ risk level of rejecting the null hypothesis $\left(H_{0}\right)$ of Gaussian distribution of the residuals, and therefore compared the statistics derived from the sample $(S)$ with $95 \%$ quantiles $(Q)$ taken from the $\chi^{2}$ with $k-3$ degrees of freedom and the $K-S$ theoretical functions. If the sample values of the two statistics, $S_{\chi^{2}}$ or $S_{D_{n}}$, were larger than the quantiles, i.e. $S / Q>1$, the $H_{0}$ hypothesis was rejected; vice versa, if $S / Q<1$, the Gaussian test was accepted.

We have tested the residual differences computed against SDSS and UCAC 2 inside R.A. strips of $2 \mathrm{~h}$ and spanning the entire decl. range. The results of the tests for different magnitude bins and for a number of different plates show that, although not strictly Gaussian, the distributions are well behaved. As a general trend, the errors in decl. are better behaved, and the R.A. error increases more toward fainter magnitudes. As an example, Figure 14 shows the results obtained by comparing GSC 2.3 coordinates against UCAC 2 on a strip of sky between 0 and $2 \mathrm{~h}$ and $-90<\delta<+50$ degrees.

If we do not bin in magnitude, the distribution does not behave like a Gaussian as would be expected from combining different Gaussian populations; however, even limiting the magnitude range to within $R_{F}=14.5-15$, which gives a total number of matched (stellar) objects of about 44,000, both tests still fail. In particular, the distribution of $\Delta \alpha \cos \delta$ diverges from the expected one, and presents a significantly larger dispersion than the $\Delta \delta$ distribution. This occurrence is indirect evidence of local systematics errors which distorts the shape of the estimated distribution function. In fact, if we restrict the sample to a single plate, and the same magnitude range (862 stars sample), both tests are better behaved, and the $H_{0}$ hypothesis is accepted, as illustrated in Figure 15.

\subsubsection{Magnitude-Dependent errors}

Morrison et al. (1996, 2001) found that positional differences of GSC-I objects matched on overlapping plate areas lead to the presence of a radial magnitude term with the origin in the plate center. Such an effect was characterized and removed from GSC-I by means of the Astrographic Catalog (Urban et al. 1998), whose magnitude limit is around 14-14.5, hence suitable to probe the faint limit of GSC-I. However, applying the same precepts to GSC-II data would fail to detect nonradial magnitude terms which we know to exist.

Monet et al. (2003) were able to use a special catalog (YS4.0, magnitude limit $V \sim 18$ ) compiled from the NPM and SPM plate scans to detect and remove magnitude-dependent residual errors during the construction of USNO-B by means of the mask method (Taff et al. 1990).

For our magnitude error analysis, we crossmatched GSC 2.3 against the adopted external catalogs using a search radius of 3 arcsec to recover the common objects and build the differences:

$\Delta \alpha \equiv\left(\alpha_{\mathrm{GSC}}-\alpha_{\mathrm{cat}}\right) \cos \delta_{\mathrm{GSC}} \Delta \delta \equiv\left(\delta_{\mathrm{GSC}}-\delta_{\mathrm{cat}}\right)$

Then, we binned the data at 0.5-mag steps and for each of such bins computed the statistics described below.

We began with estimating the global rms of the residuals as

$$
\epsilon_{\Delta \alpha}^{2}=\frac{1}{N} \sum_{i=1}^{N}\left(\Delta \alpha_{\mathrm{i}}\right)^{2}
$$

where $N$ is the number of objects (stellar or 

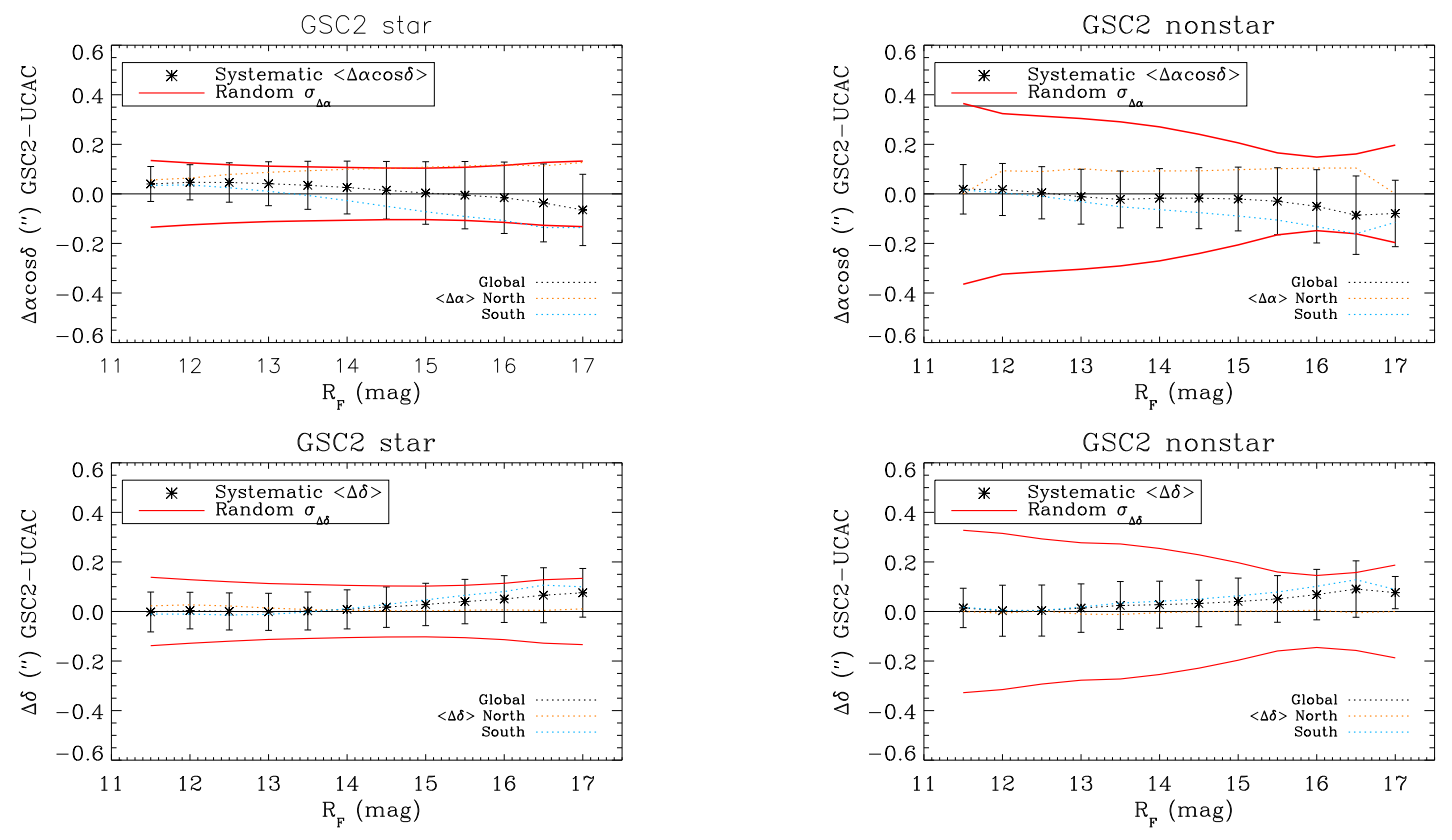

Fig. 16.- GSC 2.3 vs. UCAC 2. Astrometric residuals of GSC 2.3 sources classified as point-like objects (left panels) and extended objects (right panels). The symmetric red solid lines show the random errors, $\pm \sigma_{\Delta \alpha}, \pm \sigma_{\Delta \delta}$, as a function of the magnitude (see Equation 5). Asterisks connected by the black dotted line show the means $\langle\Delta \alpha\rangle,\langle\Delta \delta\rangle$ averaged over all the HTM regions (Equation 7), while the error bars are the standard deviation of the systematic field-to-field variations (Equation 6). The orange and blue dotted lines show the averaged $\langle\Delta \alpha\rangle,\langle\Delta \delta\rangle$ means over the northern and southern regions, respectively.

nonstellar) inside a particular magnitude bin. It is evident from this definition that $\epsilon_{\Delta \alpha}$ represents a catalog average of the total error, as it includes both systematic and random errors, and it is the quantity closest to the real astrometric error. Besides, relative astrometry over small fields (of the order of half a degree or less) is mainly affected by random errors; therefore, next we estimated the magnitude dependence of the random component of the GSC 2.3 error. For each of the $32768 \mathrm{HTM}$ regions we measured the local systematics, $\langle\Delta \alpha\rangle_{r}$, and its variance, $\sigma_{\Delta \alpha, r}^{2}$, as

$$
\langle\Delta \alpha\rangle_{r}=\frac{1}{N_{r}} \sum_{i=1}^{N_{r}} \Delta \alpha_{i}
$$

and

$$
\sigma_{\Delta \alpha, r}^{2}=\sum_{i=1}^{N_{r}} \frac{\left(\Delta \alpha_{i}-\langle\Delta \alpha\rangle_{r}\right)^{2}}{\left(N_{r}-1\right)}
$$

respectively, with $N_{r}$ being the total number of objects per $0.5 \mathrm{mag}$ bin in region $r$. Consequently, we estimated the catalog random error as the weighted mean of the standard deviations in each HTM region, i.e.,

$$
\sigma_{\Delta \alpha}^{2}=\frac{1}{N} \sum_{r=1}^{N_{R}} N_{\mathrm{r}} \sigma_{\Delta \alpha, r}^{2}
$$

where $N_{R}$ is the total number of HTM regions. Outliers due to mismatches were rejected, and GSC 2.3 entries flagged as Tycho-2 stars excluded. Also, magnitude bins with poor samples (less than 20 matched objects) or very crowded samples (more that 5000 objects) were not used in this analysis. Analogous quantities were evaluated for the residuals in decl.

The statistics in Equations 2 and 5 are summarized in Table 8, which reports the results of the comparisons with UCAC 2 for the brightto-intermediate magnitude range and with SDSS for the intermediate-to-faint range. We note that 

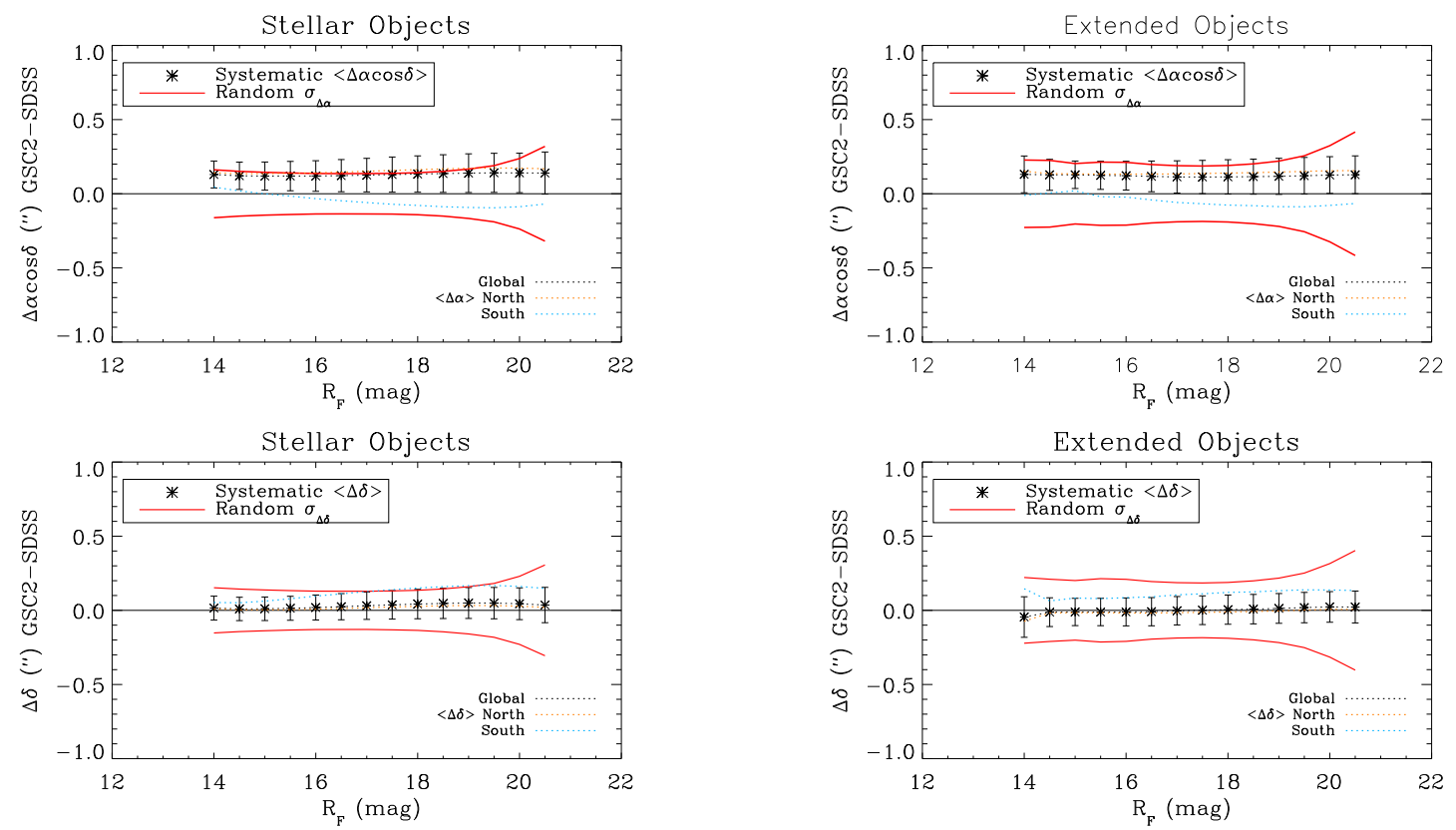

Fig. 17.- Similar to Figure 16 but for the comparison GSC 2.3 versus SDSS. Astrometric residuals as a function of the magnitude of GSC 2.3 sources classified as point-like objects (left panels) and extended objects (right panels). Same symbols as in Figure 16. Random position errors (red solid lines) are almost constant; for stellar objects $\sigma_{\Delta \alpha} \simeq \sigma_{\Delta \delta} \simeq 0.14$ down to $R_{F} \simeq 18.5$ and comparable with the small scale field-to-field systematic zero-point variations shown by the error bars. The dotted lines confirm the presence of the systematic offset between the northern/sourthern hemispheres already found at brighter magnitudes by the comparison against UCAC 2. Notice that the global mean residuals (asterisks) are very close to northern zero-points because of the small fraction of SDSS objects with negative declinations. 
the SDSS astrometry appears more precise than 2MASS at the faint limit, and therefore more representative of the actual GSC 2.3 error, although for only a portion of the sky.

The data confirm the better behavior of the point-like objects ("stars"), which attain a random error of $\sigma_{\Delta \alpha} \simeq \sigma_{\Delta \delta} \leq 0.14$ arcsec at intermediate magnitude, $14<R_{F}<18.5$, increasing up to 0.23 arcsec at the faint end $\left(R_{F} \approx 20\right)$. As expected, larger residual errors are obtained for extended objects ("nonstar"), which may include galaxies and nebulæ, as well as many blends and unresolved binaries, in particular at bright magnitudes or in crowded fields toward low galactic latitudes. In this respect, we note that the higher errors of the extended objects in the UCAC 2 comparison, as opposed to the SDSS comparison, are explained by the dominance of blended images in the former sample, whereas the latter is mostly made of truly extended objects, being the SDSS catalog confined to high galactic latitudes.

The total rms errors, $\epsilon_{\Delta \alpha}$ and $\epsilon_{\Delta \delta}$, which also include the contribution of systematic errors 2 , are of about $0.2^{\prime \prime}$ per component at intermediate magnitudes $\left(14<R_{F}<18.5\right)$, and increase to $0.3^{\prime \prime}$ down to $R_{F} \approx 20$.

An estimate of the systematic field-to-field variations in each magnitude bin can be obtained by taking the standard deviation of the $\langle\Delta \alpha\rangle_{r}$ around their averages computed over all the HTM regions, i.e.

$$
\sigma_{\langle\Delta \alpha\rangle}^{2}=\sum_{r=1}^{N_{R}} \frac{\left(\langle\Delta \alpha\rangle_{r}-\langle\Delta \alpha\rangle\right)^{2}}{\left(N_{R}-1\right)}
$$

where $\langle\Delta \alpha\rangle$ is given by

$$
\langle\Delta \alpha\rangle=\frac{1}{N_{R}} \sum_{r=1}^{N_{R}}\langle\Delta \alpha\rangle_{r}
$$

Similar expressions hold for the field-to-field variations in decl. The quantities $\sigma_{\langle\Delta \alpha\rangle}$ and $\sigma_{\langle\Delta \delta\rangle}$ are showed as the error bars in Figures 16 and 17 and they are close in size to the random errors for stellar objects in the intermediate magnitude range. The corresponding averages, i.e. Equation 7 for R.A., are indicated by asterisks in the same

\footnotetext{
${ }^{2}$ It is convenient to recall here that we chose not to remove the errors of the reference catalogs described in Section 5.1
}

Figures and represent a uniform weight mean. This is a better indicator of the GSC2.3 astrometric zero-point when compared to the straight mean taken over all of the residuals of the matched objects. For, the simple average tends to overrepresent the regions close to the galactic plane. The catalog zero point appears quite small for bright objects, as they are close to the magnitudes of the Tycho-2 stars used to calibrate the plates, while it increases up to $\sim 150$ mas in R.A. at 20th magnitude. However, a realistic estimate of the GSC 2.3 adherence to the ICRF must also account for $l o$ cal systematics, which can vary significantly from region to region; one large-scale example is the northern/southern dicotomy revealed by the orange and blue lines of Figures [16] and 17, which depict the magnitude dependence of the mean astrometric zero point in the northern and southern regions. This effect will be addressed in the next section.

\subsubsection{Spatial Dependent Errors}

To investigate spatial-dependent errors we have checked GSC 2.3 residuals versus SDSS positions of 17,507 stars along an equatorial strip with $145^{\circ} \leq \alpha \leq 205^{\circ}$ and for the magnitude range of $16.5 \leq R_{F} \leq 17.0$. From the plots of Figure 18 one can immediately note the plate-to-plate discontinuity and the gradients within each plate. In particular, the systematic zero-point scatter amounts to $0.07^{\prime \prime}$ and $0.06^{\prime \prime}$ in R.A. and decl. respectively, as derived by the standard deviation of the running mean of the residuals (red solid line), while the standard deviation of the residuals is $0.15^{\prime \prime}$ and $0.14^{\prime \prime}$.

An all-sky view of the GSC 2.3 systematic and random errors estimated by Equations 3 and 4. and utilizing 2MASS as comparison catalog, is given by the equatorial maps in Figure 19 for the magnitude range of $15<R_{F}<15.5$. The random error map shows a nonuniform distribution, with significant variations with respect to the mean values $\left(\sigma_{\Delta \alpha}\right.$ and $\left.\sigma_{\Delta \delta}\right)$ reported in Table 8. In particular, we note the poorer behavior along the galactic equator (crowding), as well as the dependence on R.A., which might indicate effects due to poorer observing conditions (seasonal effects and/or suboptimal hour angles during exposures). The higher errors at the plate corners form the peculiar speckled pattern which is most 

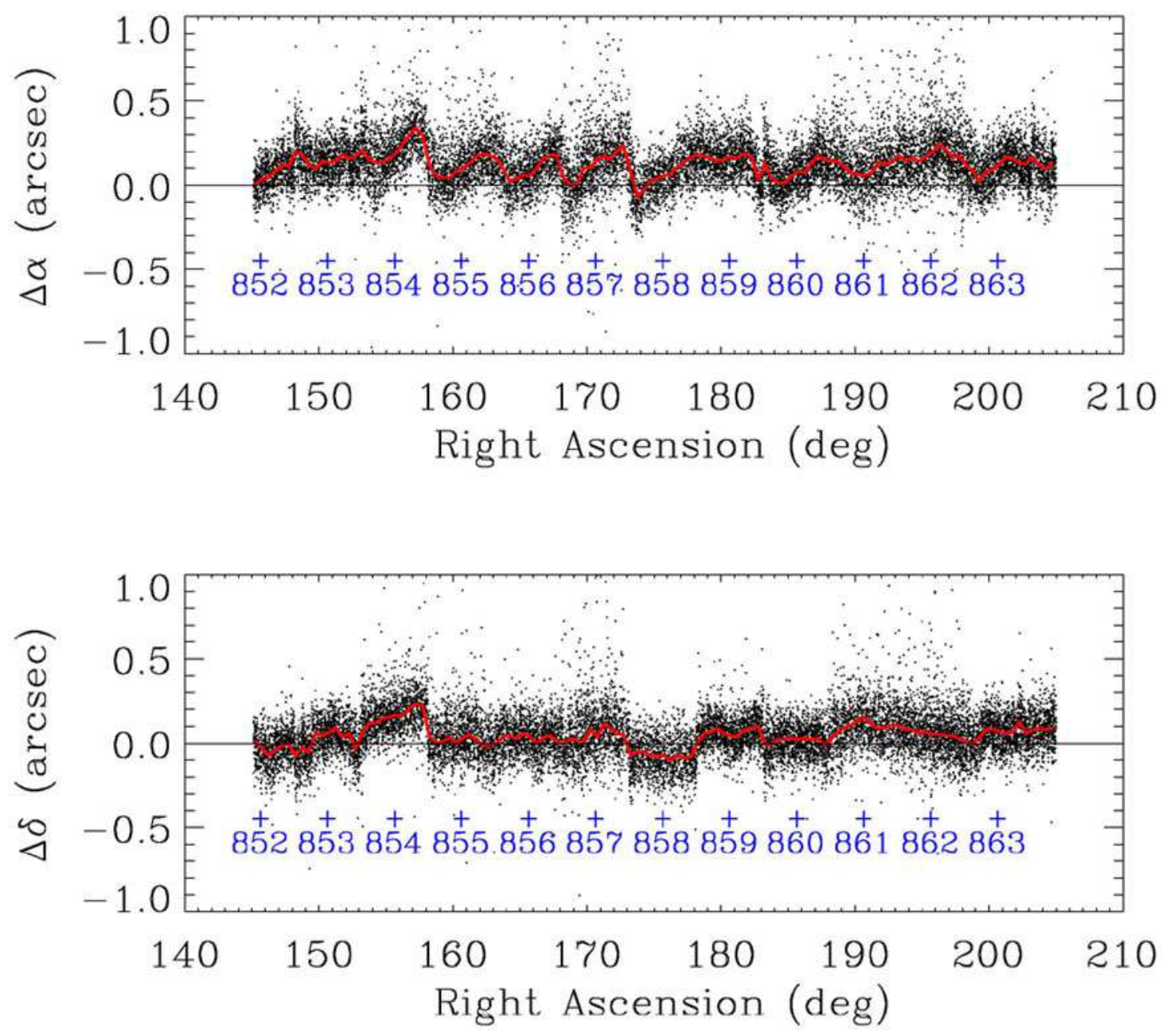

Fig. 18.- GSC 2.3 vs. SDSS. Astrometric residuals (dots), $\Delta \alpha \cos \delta$ and $\Delta \delta$, for 17,507 stars with $16.5 \leq$ $R_{F} \leq 17.0$ along an equatorial strip in the range $145^{\circ} \leq \alpha \leq 205^{\circ}$. The red solid lines are the running means of the astrometric residuals which indicate the geometric systematic errors. Here, the crosses indicate the R.A. of the centers of the POSS-II fields along the celestial equator at $\delta=0$. For this sample, the rms's of the residuals are $0.15^{\prime \prime}$ and $0.14^{\prime \prime}$ for R.A. and decl. respectively, while the zero-point scatter is $0.07^{\prime \prime}$ and $0.06^{\prime \prime}$ as derived by the standard deviation of the running mean. 

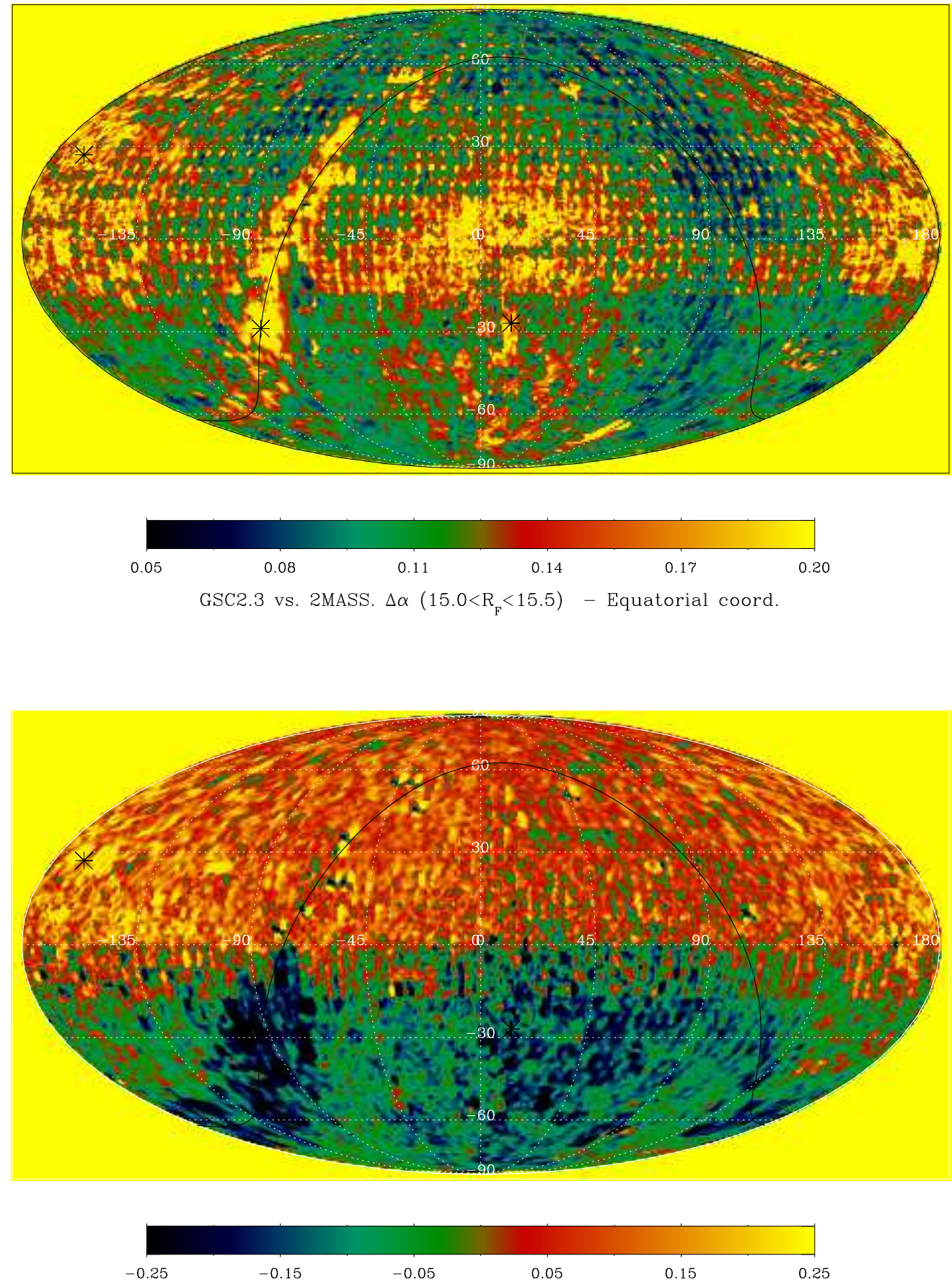

Fig. 19.- Equatorial maps of the GSC 2.3 regional random errors $\sigma_{\Delta \alpha, r}$ (top panel) and systematic errors $\langle\Delta \alpha\rangle$ (bottom panel) versus 2MASS, in the magnitude range $15<R_{F}<15.5$. The black solid lines trace the Galactic equator, while the asterisks represent the Galactic poles and the Galactic center. Similar maps are found for the decl. errors. 

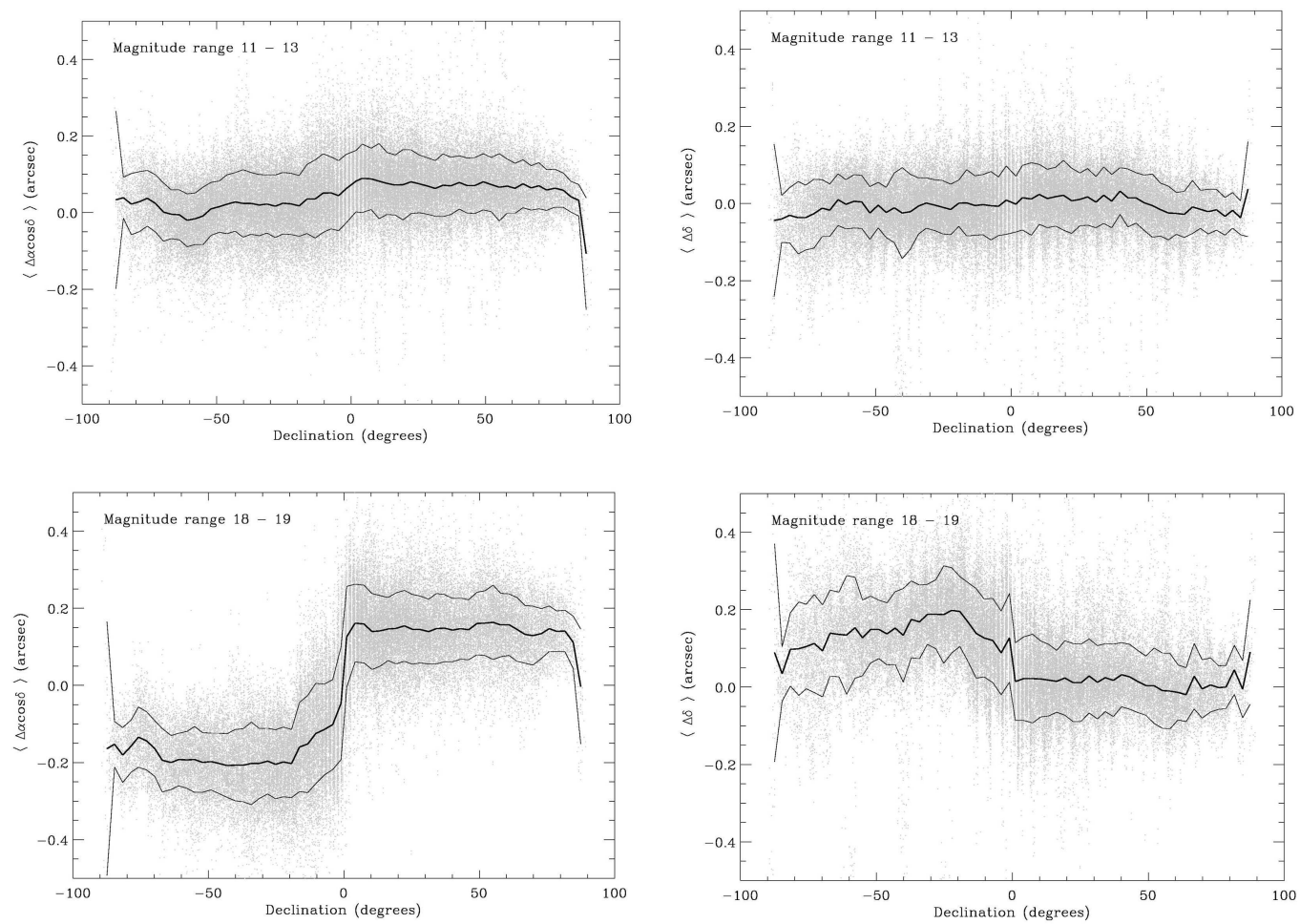

Fig. 20.- GSC 2.3 - 2MASS R.A. (left) and decl. (right) differences as a function of decl. for the $R_{F}$ magnitude ranges 11-13 (top panels) and 18-19 (bottom panels). The central solid line marks the median for the data binned in decl., while the external ones trace the rms deviations about each median.

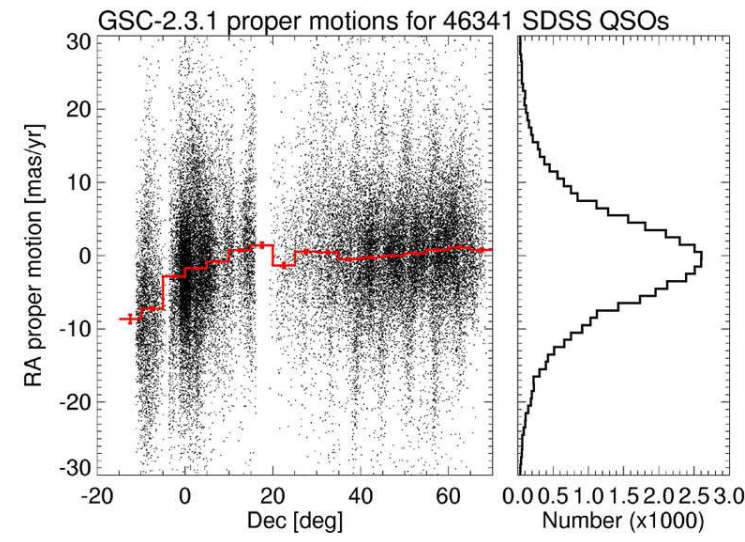

Fig. 22.- Same measurements as in Figure 21 but plotted as a function of decl. to show the systematic proper motion error in the southern hemisphere visible along the equator where the effects of the sphere mapping geometry are minimized.

The bottom panel shows a definite systematic difference between north and south, which we further analyzed. To this end, we generated platebased vector plots of the R.A. and declination differences versus 2MASS with a 1-mag step resolution and looked for possible signatures of different surveys, emulsion/filter combinations, and decl. These maps present a characteristic pattern, whose typical amplitude is negligible in the magnitude range of the reference catalog used for the astrometric solution, and increases toward fainter stars up to an average of $\sim 0.25 \operatorname{arcsec}$ (at mag $\simeq$ 20 ). Moreover, the net residual is positive or negative according to decl./survey telescope, as Figure 20 clearly shows. This sign dichotomy, which is more pronounced in R.A. than in decl., can be explained by noticing that the northeast are flipped for a northern versus southern telescope mount- 

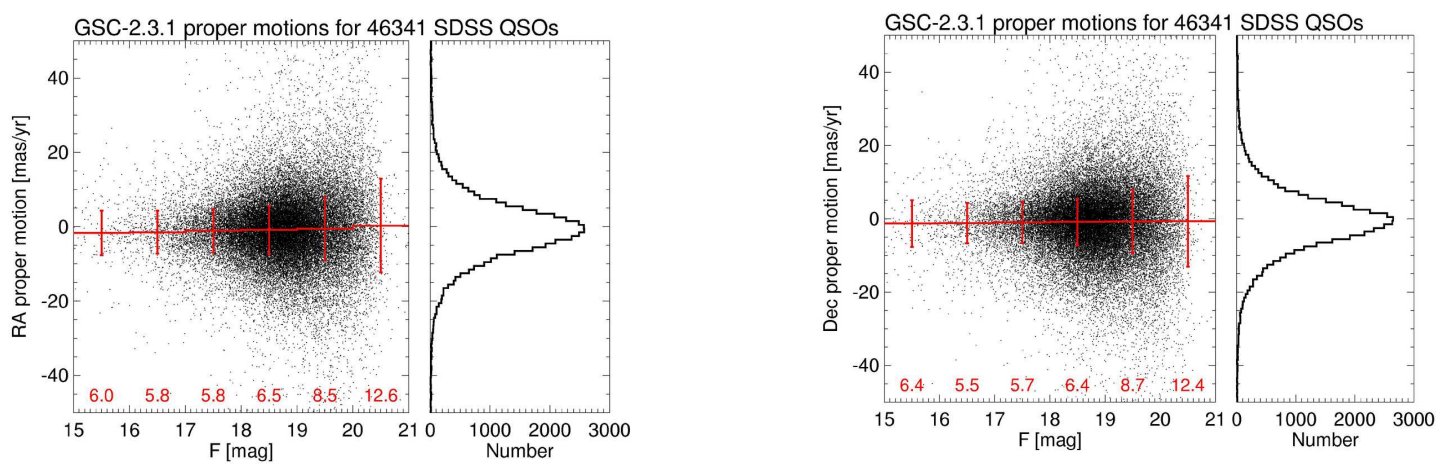

Fig. 21.- Measured proper motions of SDSS quasars in (a) R.A. and (b) decl. Since QSOs should have zero proper motion, this is a measure of the proper motion errors (see text).

ing, while, given the similarities of the observing systems utilized (telescopes, filters, plates, strategies), the systematic components have similar behavior in plate-based coordinates. Therefore, the net average astrometric residual as a function of decl. changes in sign around the equator for both coordinates. As expected, this systematic feature becomes more pronounced for fainter stars, farthest from the magnitude range of the reference catalog to which GSC 2.3 has been tied.

The analysis presented in above sections has shown that we are dealing with a complex systematic pattern of astrometric residuals, containing magnitude and spatial dependences ultimately leading down to the local nature of the observation/reduction process. It is also clear that such systematics need to be modeled locally in order to be effectively removed; this task will be addressed in the next GSC-II release. Notwithstanding, the size of the GSC 2.3 astrometric errors is such that, although they cannot be neglected for proper motion determination, they do not impair the operational use of the catalog.

\subsection{Proper Motion errors}

As mentioned in the previous section, we expected that the complex astrometric residuals would affect production of absolute proper motions when derived from simple fitting to the multi-epoch positions available in the GSC-II database. In order to test this we took a sample of quasi-stellar objects (QSOs) that had been identified in the SDSS and computed a proper motion from the GSC 2.3 measurements at all available epochs using a simple least-squares linear fit. The QSOs should have zero proper motion since the ICRS reference frame is tied to the extragalactic dynamical system. The measured proper motions are therefore an estimate of our errors. These results are shown in Figure 21. At first glance it would appear that the proper motions are determined with an rms error of 6-12 mas $\mathrm{yr}^{-1}$ (as a function of magnitude). However, if one looks at these as a function of decl. in Figure 22 we see that, whilst the northern hemisphere proper motions are determined to 6-8 mas/yr, there is a significant systematic error for the southern hemisphere which is increasing the overall error. We believe that whilst the systematic positional errors tend to cancel out among the different northern surveys, they do not for the southern ones. Therefore, although we have exported GSC2.3 proper motions from the database, these are used internally for telescope operations, and they will not be released to the scientific community until the systematic component is dealt with and minimized.

\subsection{Photometric errors}

GSC 2.3 photographic magnitudes result from the photometric plate calibrations described in Sect. 3.3.2, and from the selection criteria which defined the export catalog, as discussed in Sect. 4. Note that the GSC-II pipeline has been tuned for point-like sources and it is not optimized for galaxy photometry. Similarly to the astrometry, GSC-II photometry is affected by both positionand magnitude-dependent systematic errors. In this section, we assess the GSC 2.3 photometric ac- 


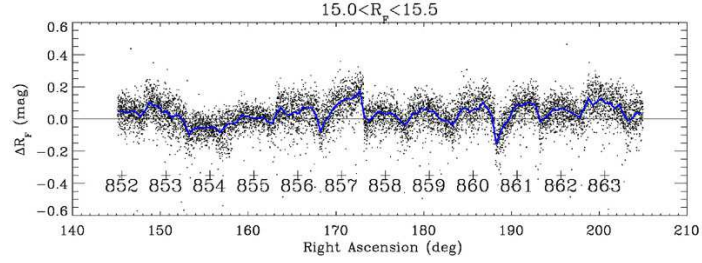

$16.5<\mathrm{R}_{\mathrm{R}}<17.0$

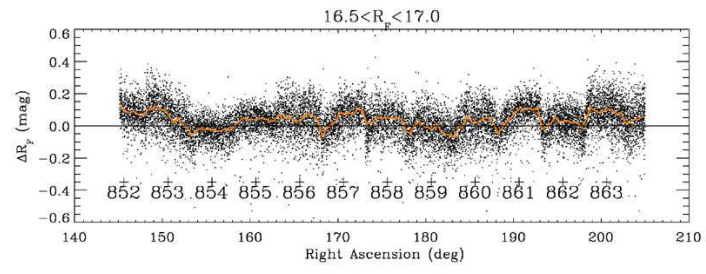

$18.0<\mathrm{R}_{\mathrm{F}}<18.5$

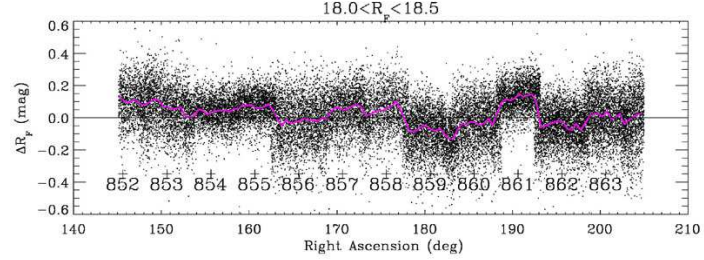

Fig. 23.- Photometric residuals, $\Delta R_{F}$, between GSC 2.3 and SDSS for a $2.5^{\circ} \times 60^{\circ}$ strip along the celestial equator. The three panels show the residuals of $9544,17,507$, and 29,709 stellar objects selected within the magnitude range $R_{F}=15.0$ $15.5,16.5-17.0$, and 17.5-18.0, respectively. The thick solid lines show the running means of the residuals. The crosses at the bottom indicates the position of the centers of the plates covering this equatorial region.

curacy via comparisons with the GSPC-II and the SDSS, once the reference magnitudes have been transformed into the natural plate bandpasses by means of the synthetic color transformations discussed in Sect. 3.3.2.

The small-scale test shown in Figure 23 gives the residuals $\Delta R_{F}$ with respect to SDSS for a strip of $2.5^{\circ} \times 60^{\circ}$ along the celestial equator covered by a dozen plates. The thick solid lines are running means which evidence a zero-point systematic error as a function of R.A., clearly correlated to the plate pattern. Peak-to-peak differences of about \pm 0.15 mag are present, although the standard deviation of the zero-point variation does not exceed 0.05-0.06 mag. The presence of a mild magnitude term is also revealed by comparing the three panels which show the residuals in the magnitude ranges $R_{F}=15.0-15.5,16.5-17.0$, and 17.5-18.0.
As expected, the random scatter around the mean clearly increases as a function of magnitude, from $\sigma_{R} \simeq 0.10 \mathrm{mag}$ to $0.14 \mathrm{mag}$ of the first and last panels, respectively.

An all-sky comparison is the one against GSPCII, which we call semi-internal as this catalog was used to calibrate the plates, even though a percentage of the matched objects did not participate in the calibration because many objects were rejected during the pipeline reduction for various reasons (e.g. color outside the valid range of the transformations, position close the plate border, etc.). Figure 24 shows the distributions of the residuals, $\Delta R_{F}$, between GSC 2.3 and GPSC-II magnitudes computed for 204,322 and 175,364 objects down to the plate limit in the northern and southern hemisphere respectively. The distribution is well behaved, with a maximum close to zero within a few hundredths of magnitudes and a standard deviation of about $0.3 \mathrm{mag}$, estimated after outliers rejection at $3 \sigma$. We note an excess of objects in the tail of the distributions with respect to a pure Gaussian, and also a slight positive skewness formed by objects having $R_{F}^{\mathrm{GSC} 2.3}<R_{F}^{\mathrm{GSPC}-\mathrm{II}}$, possibly due to unresolved binaries which result systematically brighter on the photographic plates. The residual distribution of the point-like objects is also shown in the right panel; as expected, it appears similar but with a smaller dispersion.

The bottom panels show the same statistics for objects at galactic latitudes higher than $|b|=30^{\circ}$, where the crowding is less critical. Here the limits of the photometric accuracy can be directly tested; in the case of stellar objects, dispersions attain 0.13 and $0.16 \mathrm{mag}$ in the northern and southern hemisphere respectively. The results of the statistics of all the distributions shown Fig 24 are listed in Table 9 .

An independent analysis of GSC 2.3 was done with respect to the SDSS DR5 that provides high accuracy ugriz photometry which was transformed into the GSC-II photometric system, $B_{J} R_{F} I_{N}$, and compared with the photographic magnitudes. In Tables 10, 11, and 12 we report the standard deviation of the residuals of stellar objects as a function of magnitude, in the northern and southern hemispheres. In the north, statistics are given for a $20 \%$ sample of randomly selected objects, while all the southern matches were used. 

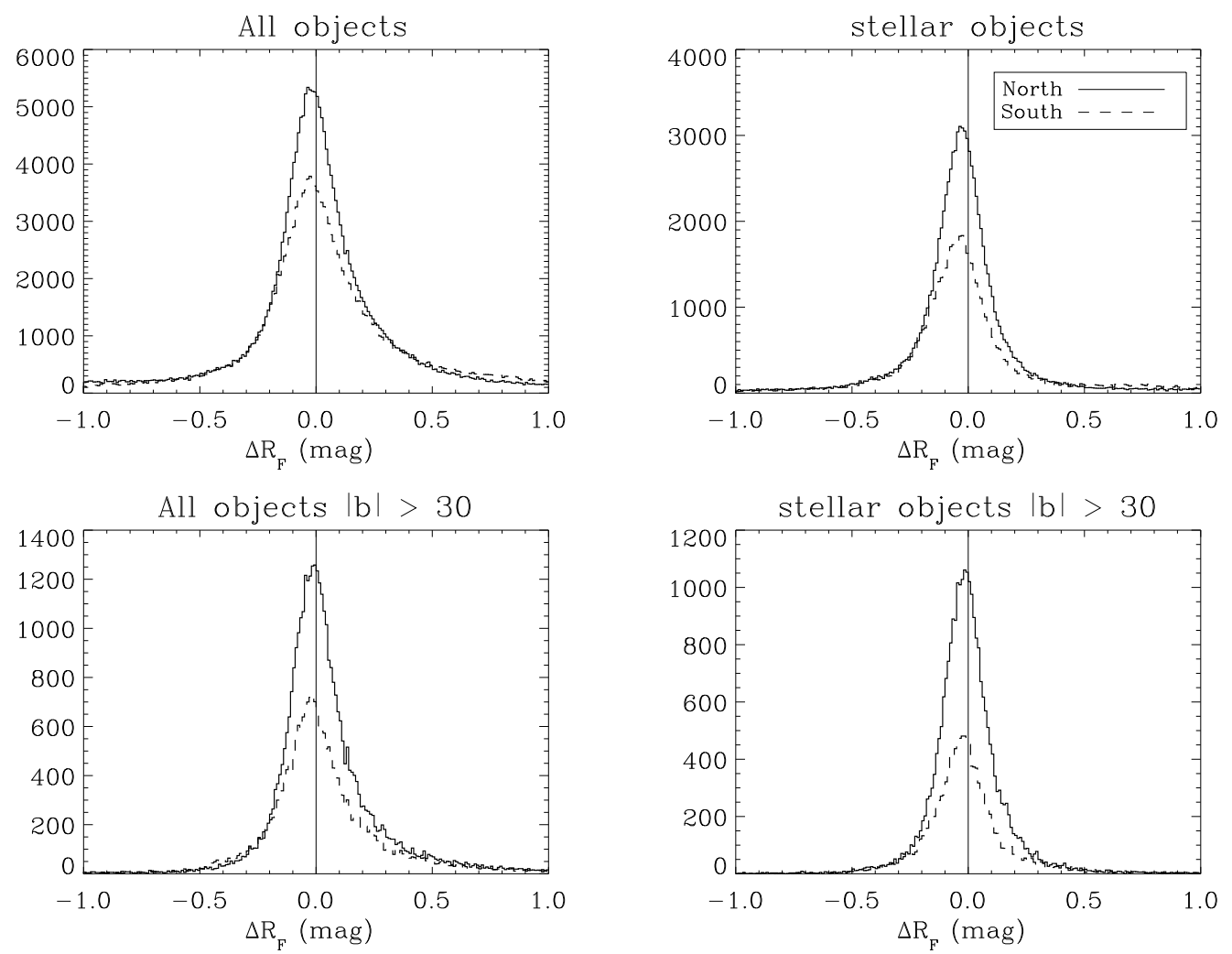

Fig. 24.- Distributions of the $\Delta R_{F}$ residuals between GSPC-2 and GSC 2.3 magnitudes for all the sky (top panels) and high galactic latitudes (bottom panels). The solid and dashed lines indicate the statistics computed in the northern and southern hemisphere, respectively, for all the objects (left panels) and stellar objects only (right panels).

The listed values were computed after rejecting $3 \sigma$ outliers. Figure 25] shows the same statistics for a subsample of 2000 objects per 0.5 mag bin, randomly drawn from the whole sample.

At intermediate magnitudes, we attain a precision of $\sigma_{\Delta B_{J}} \simeq 0.16, \sigma_{\Delta R_{F}} \simeq 0.12$, and $\sigma_{\Delta I_{N}} \simeq$ 0.13 . The errors increase for brighter and saturated stars, as well as for the faintest object up to $0.20-0.25 \mathrm{mag}$ in the second-last magnitude bin before the plate limit. Note that these values, which are averaged on a large sky area, include both random and position-dependent systematic errors (see Fig. 23).

The global zero points of the stellar objects, $\left\langle\Delta B_{J}\right\rangle, \Delta\left\langle R_{F}\right\rangle, \Delta\left\langle I_{N}\right\rangle$, averaged over the entire sky area where GSC 2.3 and SDSS DR5 overlap (about 8000 square degrees, mostly in the northern hemisphere) are usually very small, within a few hundredths of magnitude, as expected by local systematic errors which randomize on large scales.

This is not true in the case of extended objects that, as shown in Figure 26, appear systematically brighter in GSC 2.3 with respect to SDSS. The magnitude offset, $\Delta m$, increases monotonically toward brighter magnitudes up to a few magnitudes, because extended images such as galaxies or blends are less affected by the saturation of the photographic emulsion than point-like objects. In fact, $\Delta m \rightarrow 0$ for fainter sources which present densities in the linear part of the characteristic function of the photographic emulsions. Figure 26 also shows a certain number of bright sources having $\Delta m \approx 0$. Actually, these correspond to real point-like objects which have been misclassified by the classification algorithm.

Local systematic errors have been investigated 

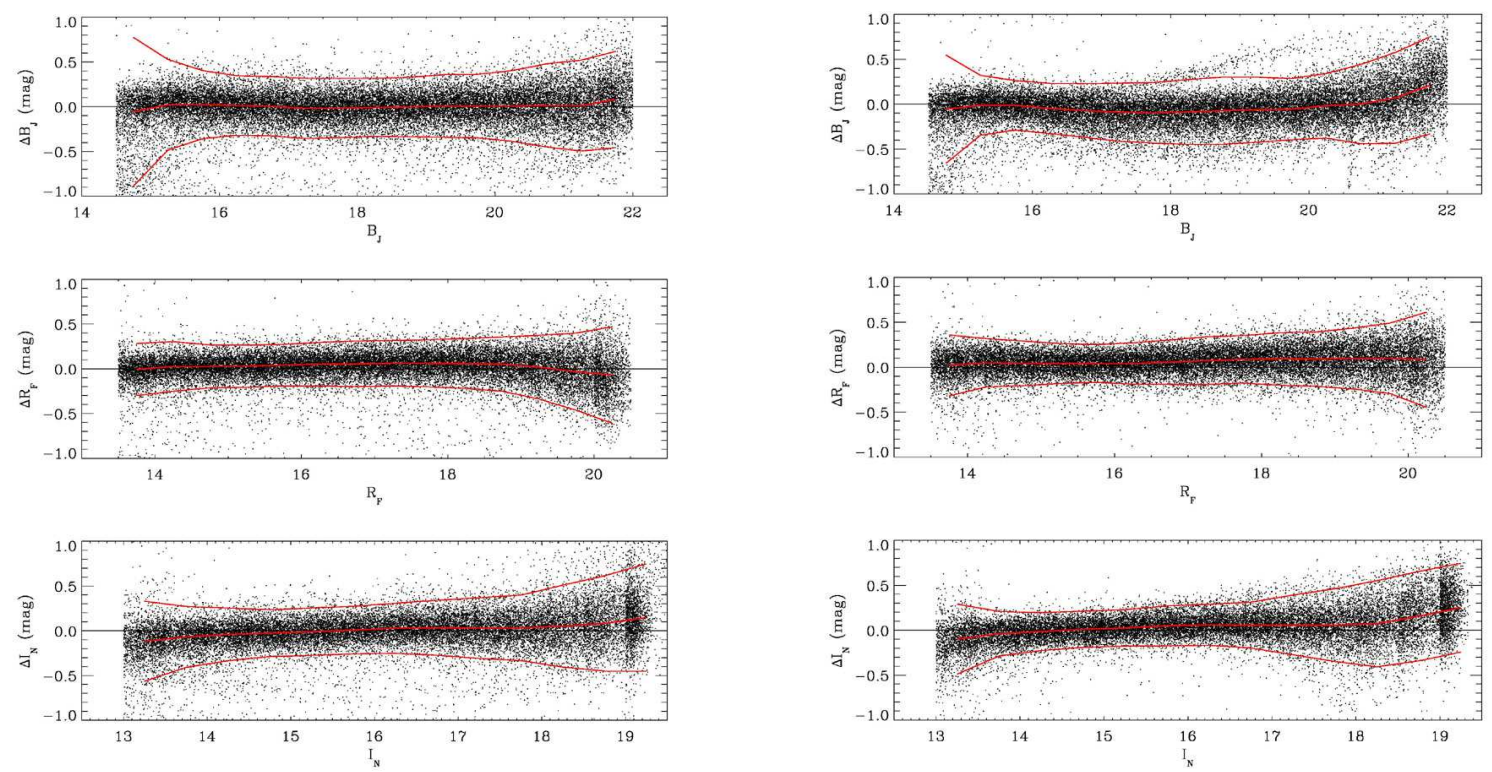

Fig. 25.- Stellar objects in the northern (left panel) and southern (right panel) hemisphere. Distributions of the $\Delta B_{J} \Delta R_{F} \Delta I_{N}$ residuals between GSC 2.3 and SDSS magnitudes for stellar objects in the northern hemisphere. Stars are uniformly distributed in magnitude, with a density of 2000 objects per 0.5 mag bin. The solid lines indicate the mean and the boundary lines at $\pm 2 \sigma$.
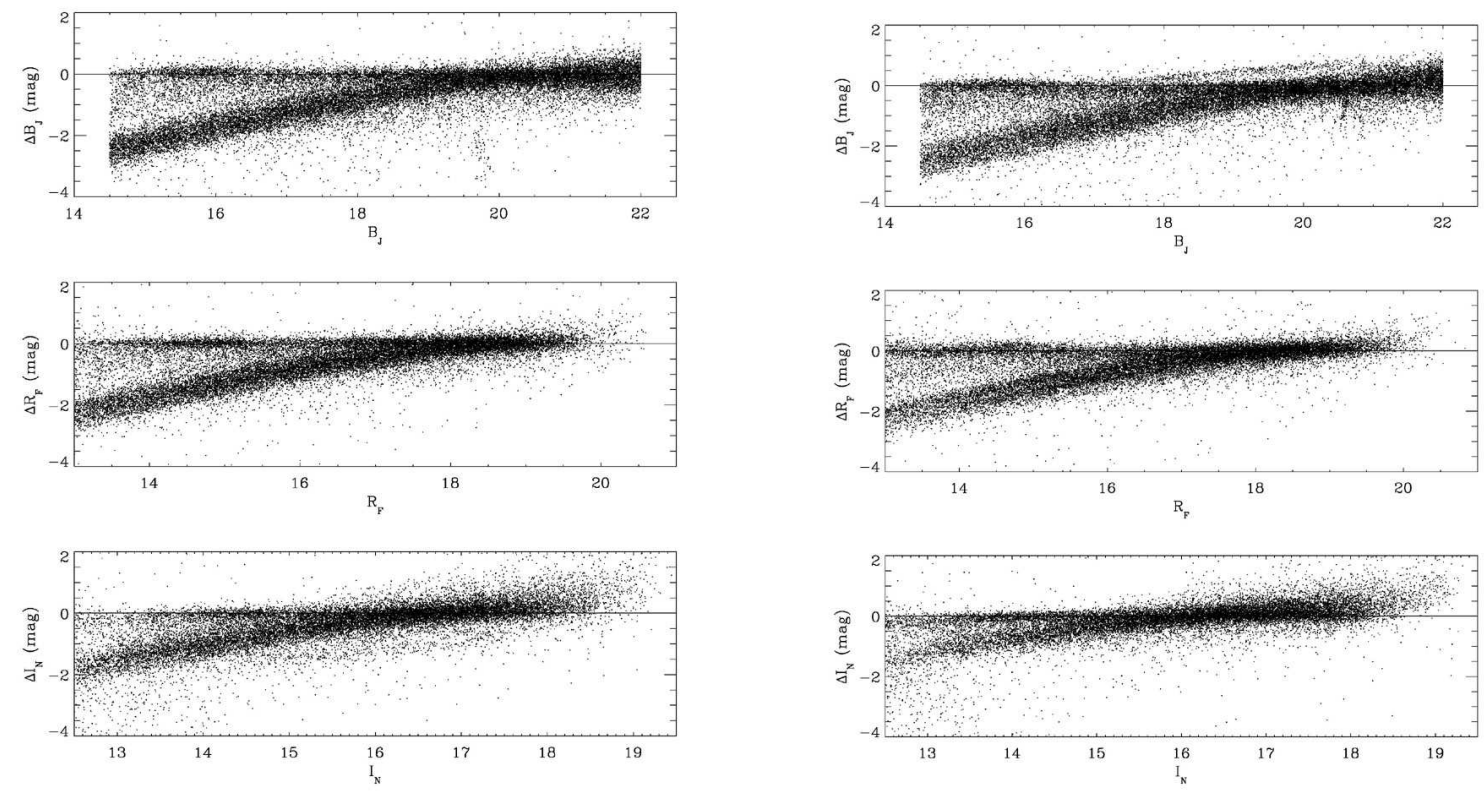

Fig. 26.- Nonstellar object in the northern (left panels) and southern hemisphere (right panels). Distributions of the $\Delta B_{J} \Delta R_{F} \Delta I_{N}$ residuals between GSC 2.3 and SDSS magnitudes for nonstellar objects in the Northern hemisphere. Stars are uniformly distributed in magnitude, with a density of 2000 objects per 0.5 mag bin. 

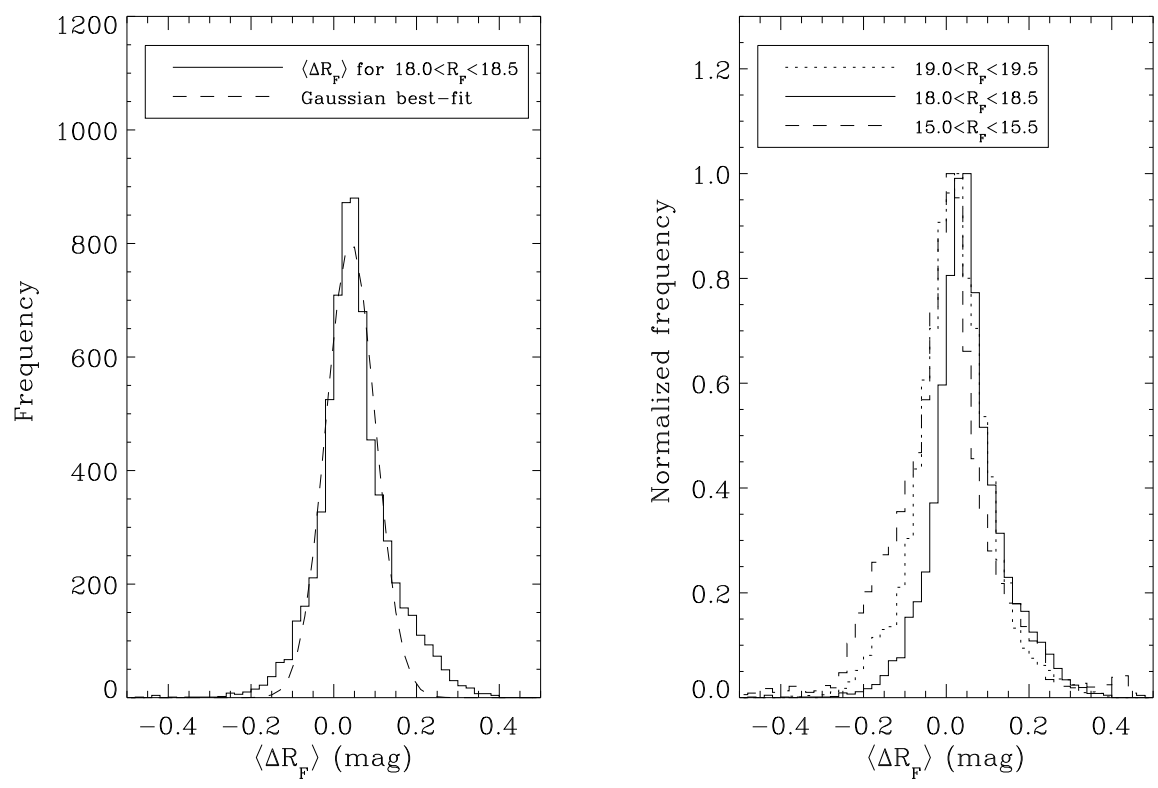

Fig. 27.- GSC 2.3 - SDSS residuals. Distribution of the mean zero point, $\left\langle\Delta R_{F}\right\rangle$, of stellar objects in the sky area $150^{\circ}<\alpha<220^{\circ}$ and $20^{\circ}<\delta<50^{\circ}$. The mean residuals have been computed within square regions of about $30^{\prime} \times 30^{\prime}$.

GSC 2.3 - SDSS residuals $\left\langle\Delta \mathrm{R}_{\mathrm{F}}\right\rangle$

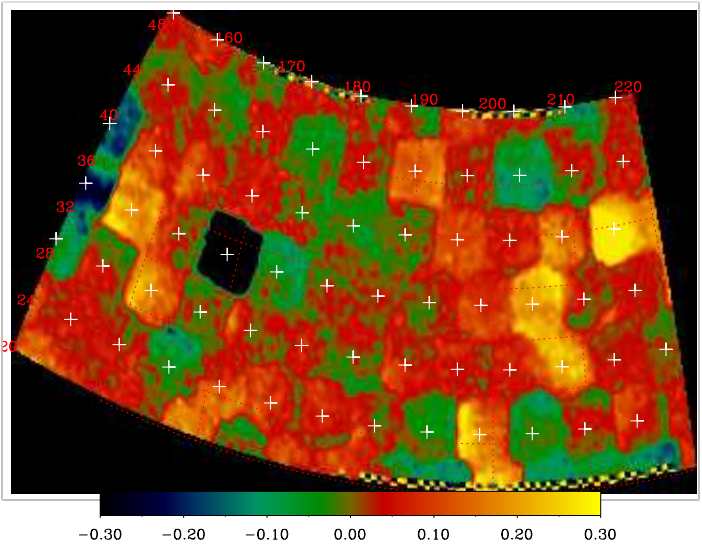

GSC 2.3 - SDSS residuals $\sigma_{\mathrm{B},}$

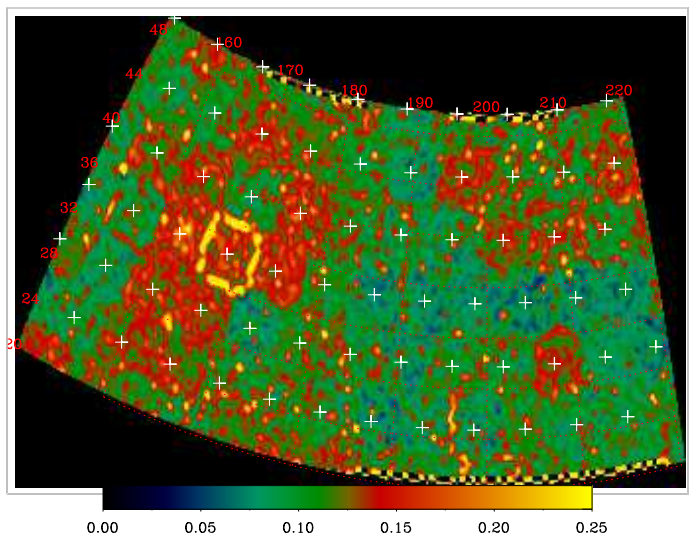

Fig. 28.- GSC 2.3 - SDSS residuals. Color map of $\left\langle\Delta R_{F}\right\rangle$ (left panel) and $\sigma_{\Delta R_{F}}$ (right panel) of stellar objects measured in the sky area $150^{\circ}<\alpha<220^{\circ}$ and $20^{\circ}<\delta<50^{\circ}$ with a spatial resolution of about $30^{\prime} \times 30^{\prime}$. The white crosses indicate the centers of the POSS-II plates. 
in details within a test area of 1700 square degrees selected at $\left(150^{\circ}<\alpha<220^{\circ}, 20^{\circ}<\delta<\right.$ $\left.50^{\circ}\right)$. There, magnitude residuals were computed in small regions of about $30^{\prime} \times 30^{\prime}$ and for different magnitude bins. The left panel of Figure 27 shows the distribution of the mean residuals, $\left\langle\Delta R_{F}\right\rangle$, of the stellar objects in the magnitude range, $18.0 \leq R_{F}<18.5$, which corresponds to the typical magnitude limit of the GSPC-II reference stars used for the photometric calibration of the plates.

The zero-point distribution appears well behaved and roughly Gaussian, although with bigger tails. It presents a mean of +0.05 mag and a standard deviation of 0.09 mag whose value represents the typical scatter of the local systematic errors that are present in the GSC 2.3 photometry.

The right panel of Figure 27 compares the zero-point distribution of three magnitude bins, $15.0 \leq R_{F}<15.5,18.0 \leq R_{F}<18.5$, and $19.5 \leq R_{F}<20.0$. The two brightest bins show similar distributions, having the same standard deviation, $\sigma_{\left\langle\Delta R_{F}\right\rangle} \simeq 0.09 \mathrm{mag}$, but a relative shift of a few hundredths of a magnitude. The faintest magnitude bin is close to the plate limit and shows a zero-point distribution which appears asymmetric and with a larger dispersion, $\sigma_{\left\langle\Delta R_{F}\right\rangle} \simeq 0.12$ mag, with respect to the previous ones. This depends on the lack on some plates of faint photometric reference stars needed to fit the nonlinear density to magnitude transformation described in Sect. 3.3.2. Note that $\sim 90 \%$ of the sky has been calibrated with photometric sequences down to $R_{F} \gtrsim 18$ and $B_{J} \gtrsim 19.5$, while for $\sim 50 \%$ of the sky the photometric sequences attain $R_{F} \gtrsim 19.5$ and $B_{J} \gtrsim 20.5$. Careful catalog users can look at the $4 \mathrm{th}, 5 \mathrm{th}$, and 6 th digits of the source status flag (3) in the export catalog in order to check if magnitudes are calibrated by means of interpolated or extrapolated transformations.

Finally, the scale length and spatial properties of the photometric systematic errors are graphically represented in Figure 28 which show maps of the photometric zero point, $\left\langle\Delta R_{F}\right\rangle$, and the local random error, $\sigma_{\left\langle\Delta R_{F}\right\rangle}$, of stellar objects with $18.0 \leq R_{F}<18.5$ over the wide test field of about 1700 square degrees. The patchy pattern shown in the left panel of Fig 28 is clearly correlated to the distribution of the photographic plates, whose centers are located on a grid having a step of $5^{\circ}$ indicated by the crosses in the figure. The zeropoint scatter is generally consistent with the 0.09 mag standard deviation derived from the distribution shown in Fig. 27. However, here we note the presence of a plate located at $\alpha \approx 170^{\circ}$ and $\delta \approx 34^{\circ}$ severely affected by large zero-point error because of the missing of a deep photometric sequence in this region.

The right panel of Figure 28 shows the maps of the local random photometric error, $\sigma_{\left\langle\Delta R_{F}\right\rangle}$, which appears quite uniform, thanks to the selection criterions described in Sect. 4.2.1. It is also interesting to note that a significantly higher error is only present on the borders of the critical plate above discussed and which overlap with the neighbor plates.

Similar error statistics have been found for the other GSC-II passbands.

\subsection{Classification}

The reliability and efficiency of the classification procedures in the GSC 2.3 are a complex interaction of many effects: plate-based imaging variations, external galaxy/star distributions, internal calibrations such as object magnitude determinations, etc. A complete performance evaluation is beyond the scope of this discussion and we limit ourselves to finding the reliability of the stellar classification because this is what is crucial for the operational uses of the GSC 2.3.

As already mentioned, we have tested our classification against various external catalogs; for this discussion we will limit ourselves to the SDSS DR5 comparison but note that the results with other catalogs are similar.

In the SDSS there are eight classifications which, in addition to star and galaxy, include cosmic rays, defects, star trails, and other artifacts. We consider here only the objects labeled as galaxy and star. For each HTM region with more than ten matches we calculated the stellar classification success probability by comparing the number of SDSS stars classified as GSC 2.3 stars with the total number of SDSS stars and similarly for galaxies and nonstars.

We limit our comparison to objects in the magnitude range $R_{F}=15-20$ as objects brighter than 15 are dominated by stars, and for objects fainter than 20 we do not have enough pixels to classify. 

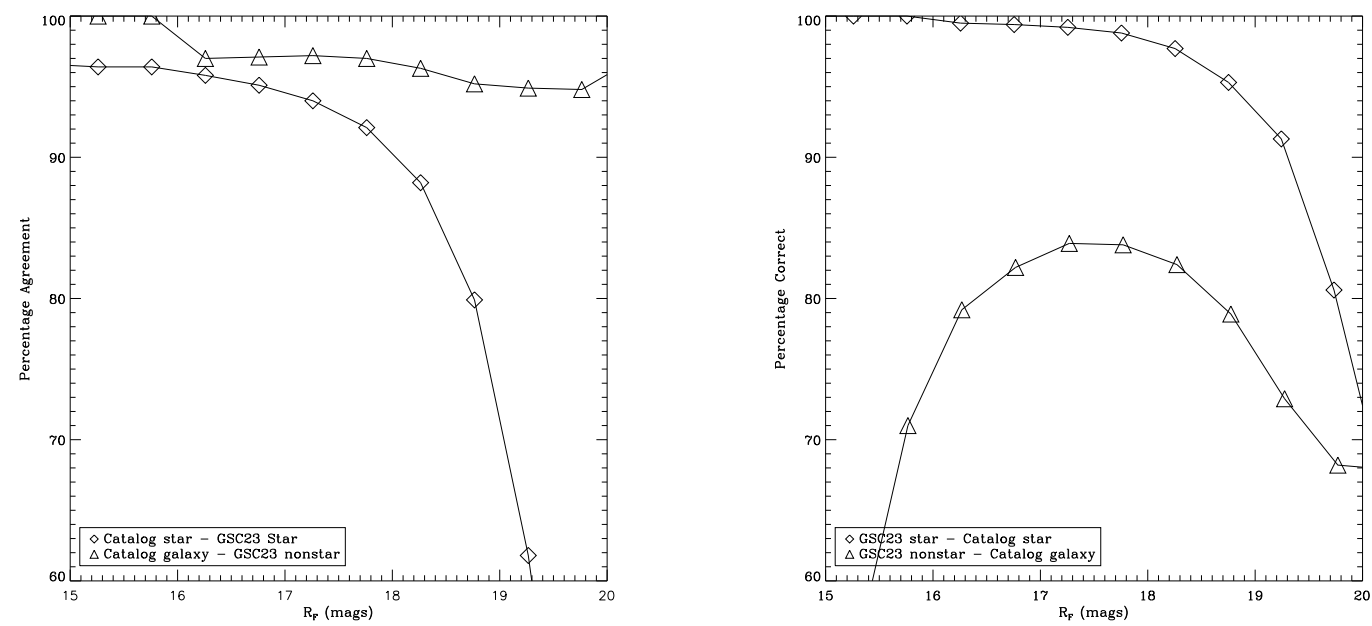

Fig. 29.- - Left panel: ratio between the number of correctly classified GSC 2.3 stars/galaxies and the total number of matched SDSS stars/galaxies, as a function of $R_{F}$ magnitude (completeness); Right panel: ratio between the number of correctly classified GSC 2.3 stars/galaxies and the total number of GSC 2.3 classified as stars/galaxies matched with SDSS as a function of $R_{F}$ magnitude (sample purity).
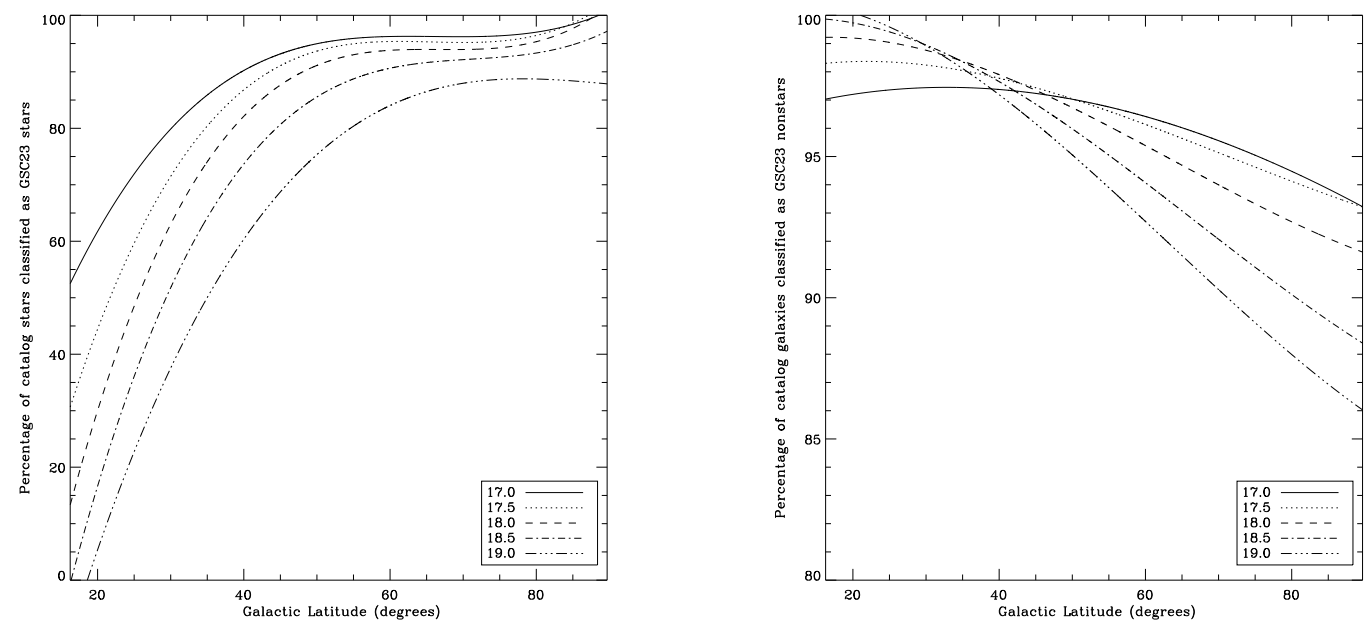

Fig. 30.- Probability of a GSC 2.3 classification agreement with the SDSS (left: stars, right: galaxies) as a function of galactic latitude, and for different magnitude ranges. 
In the left panel of Figure 29 we plot the median probability for the sampled regions as a function of $F$ magnitude. The percentage of stars correctly classified drops with magnitude from better than $90 \%$ at 18.0 to less than $50 \%$ at 20.0 , while the probability that a galaxy is classified as nonstar in the GSC 2.3 remains above $90 \%$ for all the magnitude range.

We expect a variation in galactic latitude but to examine this we restrict the magnitude range to $R_{F}=17-19$ where the number of galaxies and stars is approximately equal and the probability of a correct classification is better than $80 \%$. In Figure 30 we show the variation of the probability of a correct classification as a function of galactic latitude and magnitude. The probability of a correct classification for stars drops drastically from better than $80 \%$ at latitudes greater than 50 degrees to less than $40 \%$ for latitudes less than 20 degrees. The galaxy/nonstar agreement remains better than $80 \%$ but is worse at the poles than at the plane.

As previously stated, the purity of the stellar sample, i.e. that an object classified as a star is indeed a star, is important for guide stars use in telescope operations. This leads to the strategy of adopting a classifier that conservatively classifies objects as "stars", but accurately classifies extended objects as "non-stellar". These properties are reflected in Figure 30, the low "accuracy" of the stellar classifier at faint magnitudes is a result of the classifier being conservative, which does not affect operations negatively as the number of stars is ever increasing at fainter magnitudes.

The right panel of figure 29 plots the reliability or purity of the stellar classification as a function of magnitude. This is calculated by comparing the number of objects classified as stars both in SDSS and GSC 2.3 to the total number of GSC 2.3 stars. From this we can see that our stellar classification is reliable at the $90 \%$ level to fainter than 19th magnitude.

\subsection{Completeness}

The magnitude limit of the Schmidt plates on which the GSC-II is based is nominally 20.5 in $R_{F}$, so that the catalog is expected to be complete to at least $R_{F}=20$. This completeness limit is confirmed at high galactic latitudes using the SDSS
(Drimmel et al. 2007, in preparation) along an equatorial strip covering more the 300 square degrees; more than $99 \%$ of the SDSS point sources are successfully matched to a GSC 2 object down to magnitude $R_{F} \approx 20$ using a search radius of 5 ", while more than $97 \%$ of the SDSS extended objects were matched at magnitude $R_{F}=19$. However, in very crowded fields near the Galactic plane the GSC-II may suffer incompleteness at magnitudes brighter than $R_{F}=20$ due to crowding effects. For this reason completeness in the GSC-II is not uniform over the sky.

To assess the completeness limit for each HTM region we use a model-independent approach based on the assumption that, for a small magnitude interval (1-1.5 mag), the slope of the logarithmic magnitude distribution, $\partial \log n(m) / \partial m$ $(n(m)$ being the object field density at magnitude $m$, is nearly constant and positive, consistent with the expectation that the number of objects increases at ever fainter magnitudes. The constancy of the slope is clearly an approximation; for galaxies this assumption is very nearly true for sufficiently large areas of the sky, while for stars the slope changes slowly. In any case, these assumptions are reasonable for the magnitudes we are considering, whether we are considering stars or galaxies or all objects together. Thus, we bin the objects in $0.5 \mathrm{mag}$ bins to magnitude $R_{F}=20$ for each HTM region and apply the following procedure to estimate the completeness limit of each HTM region.

First, the magnitude bin containing the maximum number of stars is adopted as a first guess of the last (faintest) complete bin. This guess is checked by performing a linear fit to the log counts of the previous two bins. If this presumed last complete bin is the last magnitude bin, or if the fainter bins are empty, then a simple completeness test is performed: if the count in this bin agrees to within two times the Poisson error of the count predicted from the linear fit, then the maximum magnitude of this bin is adopted as the magnitude limit. For the large majority of regions more than 30 degrees from the Galactic plane, the last bin $\left(R_{F}=19.5\right.$ to 20$)$ meets this criterion and the limiting magnitude of the counts $\left(R_{F}=20\right)$ is adopted as the completeness limit.

If the above criterion is not satisfied then a refined estimate of the last complete bin is made by 


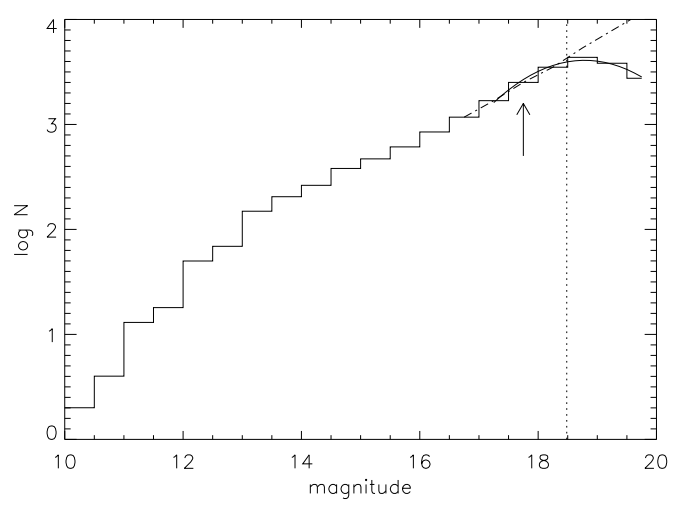

Fig. 31.- Example of determination of the magnitude limit. The histogram shows the raw GSC 2 object counts from HTM region S2200131, as a function of $R_{F}$ magnitude. The vertical arrow indicates the last complete bin, the dash-dotted line shows the expected magnitude distribution, the thick solid curve represents the actual magnitude distribution, and the dotted line indicates the determined $90 \%$ completeness limit.

requiring that 1) the slope of the log-magnitude distribution decreases less than $30 \%$ over $1 \mathrm{mag}$, or that the count is not less than $2 \sigma$ of the count predicted from a linear fit to the previous two magnitude bins, and 2) that the local slope of the logmagnitude distribution is positive. The slope and change in slope of the counts are evaluated using a second-order polynomial that fits the counts in the hypothesized last complete bin and the previous two bins. We also require that the slope of the above-mentioned linear fit be positive. If any of these conditions are not satisfied, the previous (brighter) magnitude bin is tested. This procedure is repeated until a magnitude bin is found that satisfies the above conditions. Note that if the actual count in the presumed last complete bin exceeds the predicted counts it is taken as being the last complete bin.

Once the last complete bin is determined, we estimate the expected counts after the last complete bin. The expected counts are based on a local linear fit to the faintest part of the logarithmic magnitude distribution determined to be complete, though now based on three magnitude bins, including the last complete bin if the count in this

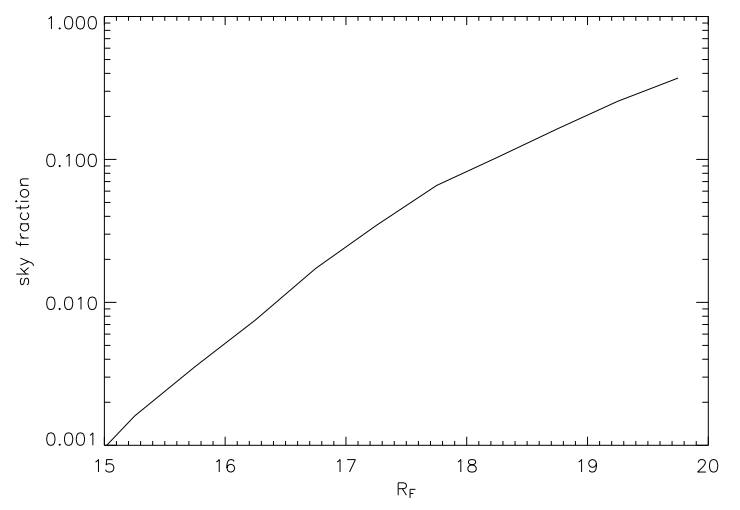

Fig. 32.- Fraction of sky less than $90 \%$ complete, as a function of $R_{F}$ magnitude.

bin does not differ more than $2 \sigma$ from the estimated count based on the previous two bins. If the last complete bin does not satisfy this criteria then the previous three bins are used in the linear fit. This linear model is our expected logmagnitude distribution, that is $\log n_{e}$.

A check is then made to see if any fainter bins are within 2 sigma of our expected magnitude distribution, or exceeds the predicted counts. If so, the faintest such bin is considered as our faintest complete bin, though we retain the expected magnitude distribution already found. This check is necessary only to handle a few regions with particularly "noisy" counts. Indeed, the complexity of the procedure described above was necessitated to assure that a last complete bin could be robustly determined for all HTM regions, including the relatively few pathological cases that can occur in very crowded regions where the photometric calibration was problematic.

We now make a more refined estimate of the completeness limit using a second-order polynomial to describe the part of the log-magnitude distribution where incompleteness sets in, from the bin preceding the last complete bin, and up to two bins beyond the last complete bin, using only bins with nonzero counts. Thus, since at this point in the algorithm the last complete bin does not coincide with the last nonempty bin, the polynomial is fit to three or more points. We adopt this polynomial as the measured log-magnitude distribution, $\log n$. To determine to what limiting magnitude 


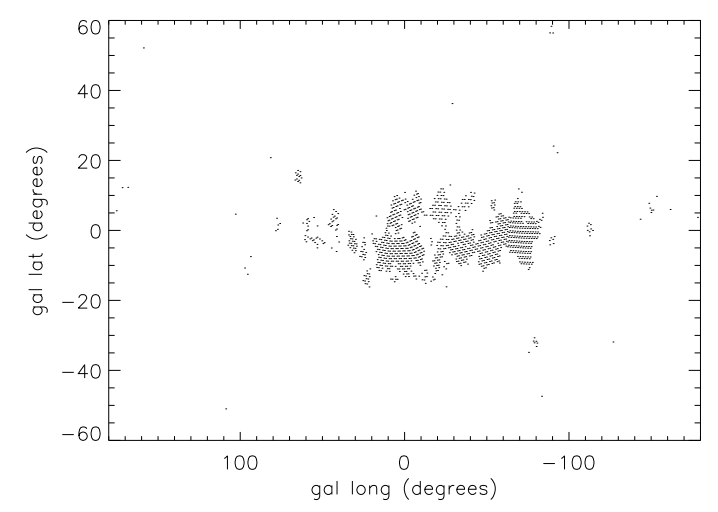

Fig. 33.- Position on the sky of the HTM regions showing less than $90 \%$ completeness at magnitude $R_{F}=18.5$.

the counts are, say, $90 \%$ complete, we find the root to the equation

$$
\log n(m)-\log n_{e}(m)-\log (.9)=0,
$$

that is, the magnitude at which the ratio of the measured and expected counts, $n / n_{e}$, is equal to 0.9 .

Figure 31 shows an example of the application of this procedure to the counts of a region showing evidence of incompleteness.

Using the above procedure we estimate the $90 \%$ completeness limit for each HTM region using the total object counts. As a statistical measure of completeness we show in figure 32 the fraction of sky that is less than $90 \%$ complete as a function of magnitude. For example, $10 \%$ of the sky shows incompleteness at magnitude $R_{F}=18.5$ or brighter, while at 17 th magnitude $3 \%$ of the sky shows evidence of incompleteness.

Incompleteness at magnitudes brighter than about $R_{F}=19$ is strictly related to the object field density, as in this case incompleteness arises not from detector (plate) sensitivity, but from the photographic plates being unable to distinguish objects from the "background" light of fainter objects whose images are blended due to the finite resolution of the plates.

Figure 33 shows the location on the sky of the HTM regions showing incompleteness at magnitudes brighter than $R_{F}$ 18.5.

\section{Conclusions and future work}

In Table 13, we compare the requirements provided in the GSC-II implementation planoriginally foreseen to reach magnitude 18 in $V-$ with the actual performance of the current version of the catalog down to the same magnitude limit. In all items we have met or exceeded the specifications except for the proper motions.

Table 14] summarizes the main characteristics of GSC 2.3, which essentially superseeds the requirements in that it reaches 2-3 mag depeer. The astrometric and photometric errors reported in the table are derived from those of Tables 8 and 11$]$ and positional uncertainties are obtained by summing in quadrature the error in each coordinate. A consideration of the global statistics shows that even with the inclusion of fainter objects we are able to meet the original specifications.

Whilst no further improvements are required (or funded) for its use in $H S T$ operations, we intend to continue development to produce the best possible catalog for scientific as well as operational uses. Our future plans include an astrometric recalibration using the UCAC 3 and applying a magnitude-dependent correction; this should not only reduce the absolute positional errors but amend the systematic error that is affecting the computed proper motions. A photometric re-reduction of all plates will also benefit from the improved overall quality of the available calibrating sequences. Finally, we plan to reclassify the objects splitting the nonstar classification into galaxy and blend as well as better determine the magnitudes of galaxies using an algorithm that does not assume a stellar profile (Petrosian et al. 2007).

As of cycle 15, GSC 2.3 is the guide catalog for $H S T$, and it is a reference catalog for the VLT and Gemini adaptive optics programs, where real guide stars are still preferred to laser "guide stars"; it has also been provided to the Chandra, Galex, XMMNewton and Swift missions. In the preparation for the Gaia mission, the current catalog version is used as a snapshot of what Gaia is expected to observe (Drimmel et al. 2006) and as a base for the Initial Gaia Source List being compiled by OATo for the Gaia data reduction. Finally, GSCII future release(s) will be at the heart of the Astrometric Support System of the very ambitious 
Large Sky Area Multi-Object Fiber Spectroscopic Telescope (LAMOST) undertaken by the Chinese Academy of Science, and will be part of the guide system for JWST (Spagna 2001; Stys and Kriss 2003).

In addition to the operational uses of the catalog itself, the GSC-II database has also been mined for many scientific studies, such as planetary nebulae (Kerber et al. 2004), young open clusters (Sciortino et al. 2000), halo white dwarfs (Carollo et al. 2006), peculiar objects (Mirabel et al. 2001; Carollo et al. 2002), X-ray pulsars (Panzera et al. 2003), and the structure and kinematics of stellar populations of our Galaxy (Vallenari et al. 2006; Kinman et al. 2007).

Finally, it is worth going back to the density map presented in Figure 13, as it does illustrate GSC 2.3: it provides accurate positions, magnitudes in three bands, and stellar classification for $4 \pi$ steradians to levels of completeness commensurate with the underlying stellar density. Future versions extracted from the GSC-II II database will only improve on this situation, including proper motions and increasing the catalog accuracy as a result of improvements in reference catalogs and reduction procedures.

\section{Acknowledgements}

The Guide Star Catalog II is a joint project of the STScI and the OATo. Space Telescope Science Institute is operated by the Association of Universities for Research in Astronomy, for the National Aeronautics and Space Administration under contract NAS5-26555. The Osservatorio Astronomico di Torino is operated by the Italian National Institute for Astrophysics (INAF).

Additional support was provided by the European Southern Observatory, Space Telescope European Coordinating Facility, the International GEMINI project, and the European Space Agency Astrophysics Division.

The DSS was produced at the Space Telescope Science Institute under U.S. Government grant NAG W-2166. Additional support was provided by Beijing Astronomical Observatory, Canadian Astronomical Data Center, Centre de Donnee Stellaire, European Southern Observatory, and the National Astronomical Observatory Japan.
The DSS images are based on photographic data obtained using the Palomar Oschin Schmidt Telescope and the UK Schmidt Telescope. The plates were processed into compressed digital form with the permission of these institutions. The National Geographic Society-Palomar Observatory Sky Atlas (POSS-I) was made by the California Institute of Technology with grants from the National Geographic Society. The POSS-II was made by the California Institute of Technology with funds from the National Science Foundation, the National Aeronautics and Space Administration, the National Geographic Society, the Sloan Foundation, the Samuel Oschin Foundation, and the Eastman Kodak Corporation. The UK Schmidt Telescope was operated by the Royal Observatory Edinburgh, with funding from the UK Science and Engineering Research Council (later the UK Particle Physics and Astronomy Research Council), until June 1988, and thereafter by the AngloAustralian Observatory. The blue plates of the southern Sky Atlas and its Equatorial Extension (together known as the SERC-J), the near-IR plates (SERC-I), as well as the Equatorial Red (ER), and the Second Epoch [red] Survey (SES) were all taken with the UK Schmidt telescope at the AAO.

Partial support for the preparation of this publication was provided by INAF through PRIN2005 grant (CRA 1.06.08.02) to OATo. Also, B.D. and M.G.L. acknowledge the continuous support from STScI through the Institute's Visitor Program.

Of the many individuals who gave us their support and assistance throughout the realization of the GSC-II Project, we are especially grateful to Piero Benvenuti, Bob Brucato, George Djorgovski, Rodger Doxsey, Fabio Favata, Attilio Ferrari, Nathalie Fourniol, Riccardo Giacconi, Fred Gillette, Jim Gray, Helmut Jenkner, Nigel Hambly, Malcolm Hartley, Harvey MacGillivray, Giuseppe Massone, David Morgan, Benoit Pirenne, Marc Postman, Phil Puxley, Mike Read, Neill Reid, Alex Szalay, Sue Tritton, Fred Watson, Andreas Wicenec, and Peredur Williams.

\section{REFERENCES}

Adelman-McCarthy, J. K., Agüeros, M. A., Allam, S. S., Anderson, K. S. J., Anderson, S. F., Annis, J., Bahcall, N. A., Bailer-Jones, C. A. L., 
Baldry, I. K., Barentine, J. C., and 144 coauthors 2007, ApJS, 172, 634

Beard, S. M., MacGillivray, H. T., and Thanisch, P. F. 1990, MNRAS, 247, 311

Bucciarelli, B., García Yus, J., Casalegno, R., Postman, M., Lasker, B. M., Sturch, C., Lattanzi, M. G., McLean, B. J., Costa, E., Falasca, A., Le Poole, R., Massone, G., Potter, M., Rosenberg, A., Borgman, T., Doggett, J., Morrison, J., Pizzuti, A., Pompei, E., Rehner, D., Siciliano, L., and Wolfe, D. 2001, A\&A, 368, 335

Bucciarelli, B. and et al. 2006, Guide Star Photometric Catalog V2.4, VizieR Online Data Catalog II 272

Bushouse, H. and Simon, B. 1994, in ASP Conf. Ser. 61, Astronomical Data Analysis Software and Systems III, ed. D.R. Crabtree, R.J. Hanisch, and J. Barnes (San Francisco, CA: ASP), 339

Carollo, D., Bucciarelli, B., Hodgkin, S. T., Lattanzi, M. G., McLean, B., Morbidelli, R., Smart, R. L., Spagna, A., and Terranegra, L. 2006, A\&A, 448, 579

Carollo, D., Hodgkin, S. T., Spagna, A., Smart, R. L., Lattanzi, M. G., McLean, B. J., and Pinfield, D. J. 2002, A\&A, 393, L45

Cornuelle, C. S., Aldering, G., Humphreys, R. M., Larsen, J., and Cabanela, J. 1997, The APS Catalog of the POSS I, Image Database, and Luyten Proper Motion Catalog, in.ASP Conf. Ser. 127, Proper Motions and Galactic Astronomy, ed. R. M. Humphreys (San Francisco, CA: ASP), 55

Djorgovski, S. G., Carvalho, R. R., Gal, R. R., Odewahn, S. C., Mahabal, A. A., Brunner, R., Lopes, P. A. A., and Kohl Moreira, J. L. 2003, The digital Palomar observatory sky survey (DPOSS): general description and the public data release, Bull. Astron. Soc. Braz., 23, 197

Drimmel, R., Bucciarelli, B., Lattanzi, M. G., Spagna, A., Jordi, C., Robin, A. C., Reylé, C., and Luri, X. 2005, What Gaia Will See: All-Sky
Source Counts from the GSC2, in The ThreeDimensional Universe with Gaia, ESA Special Publication 576, ed. C. Turon, K. S. O'Flaherty, and M. A. C. Perryman, (Noordwijk: ESA), 163

Drimmel, R., Spagna, A., Bucciarelli, B., Lattanzi, M., and Smart, R. 2006, Mem. Soc. Astron. Ital., 77, 1172

Graham, A. W., Driver, S. P., Allen, P. D., \& Liske, J. 2007, MNRAS, 378, 198

Gunn, J. E. and Stryker, L. L. 1983, Stellar spectrophotometric atlas, wavelengths from 3130 to $10800 \AA$, ApJS, 52, 121

Hambly, N. C., MacGillivray, H. T., Read, M. A., Tritton, S. B., Thomson, E. B., Kelly, B. D., Morgan, D. H., Smith, R. E., Driver, S. P., Williamson, J., Parker, Q. A., Hawkins, M. R. S., Williams, P. M., and Lawrence, A. 2001, MNRAS, 326, 1279

Høg, E., Fabricius, C., Makarov, V. V., Urban, S., Corbin, T., Wycoff, G., Bastian, U., Schwekendiek, P., and Wicenec, A. 2000, A\&A, 355, L27

Infante, L. 1993, Counts and Colours from the NGP CFHT Faint Galaxy Survey, in ASP Conf. Ser. 51, Observational Cosmology, ed. G. L. Chincarini, A. Iovino, T. Maccacaro, and D. Maccagni (San Francisco, CA: ASP), 304

Irwin, M. and McMahon, R. 1992, APM Northern Sky Catalogue, in IAU Commission on Instruments, vol. 2, 31

Ivezić, Ž., Lupton, R. H., Schlegel, D., Boroski, B., Adelman-McCarthy, J., Yanny, B., Kent, S., Stoughton, C., Finkbeiner, D., Padmanabhan, N., Rockosi, C. M., Gunn, J. E., Knapp, G. R., Strauss, M. A., Richards, G. T., Eisenstein, D., Nicinski, T., Kleinman, S. J., Krzesinski, J., Newman, P. R., Snedden, S., Thakar, A. R., Szalay, A., Munn, J. A., Smith, J. A., Tucker, D., and Lee, B. C. 2004, Astron. Nachr., 325, 583

Jenkner, H., Lasker, B. M., Sturch, C. R., McLean, B. J., Shara, M. M., and Russel, J. L. 1990, AJ, 99, 2082 
Kerber, F., Mignani, R. P., Pauli, E.-M., Wicenec, A., and Guglielmetti, F. 2004, A\&A, 420, 207

Kinman, T. D., Cacciari, C., Bragaglia, A., Buzzoni, A., \& Spagna, A. 2007, MNRAS, 375, 1381

Klemola, A. R., Hanson, R. B., and Jones, B. F. 1994, Lick NPM program: NPM1 Catalog and its applications, in Galactic and Solar System Optical Astrometry, ed. L. V. Morrison and G. F. Gilmore (Cambridge: Cambridge University Press), 20

Konig, A. 1962, Astronomical Techniques, (Chicago, IL: Chicago University Press)

Kunszt, P. Z., Szalay, A. S., Csabai, I., and Thakar, A. R. 2000, The Indexing of the SDSS Science Archive, in ASP Conf. Ser. 216, Astronomical Data Analysis Software and Systems IX, ed. N. Manset, C. Veillet, and D. Crabtree (San Francisco, CA: ASP), 141

Kunszt, P. Z., Szalay, A. S., and Thakar, A. R. 2001, The Hierarchical Triangular Mesh, in Mining the Sky, ed. A. J. Banday, S. Zaroubi, and M. Bartelmann (Berlin: Springer-Verlag), 631

Laidler, V. G., Greene, G. R., Ray, K., Evzerov, A., and Lasker, B. M. 1994, GAMMA-A NewHigh-speed Microdensitometer Built on a PDS Substrate, BAAS, 26, 897

Lasker, B. M., Greene, G. R., Lattanzi, M. J., McLean, B. J., and Volpicelli, A. 1998, DSS-II and GSC-II: STScI All-Sky Image and Catalog Databases, in Astrophysics and Algorithms; a DIMACS Workshop on Massive Astronomical Data Sets

Lasker, B. M. and Lattanzi, M. G. 1994, The Guide Star Catalog II Construction Project: Implementation Plan, Technical Note

Lasker, B. M., Sturch, C. R., Lopez, C., Mallamas, A. D., McLaughlin, S. F., Russell, J. L., Wisniewski, W. Z., Gillespie, B. A., Jenkner, H., Siciliano, E. D., Kenny, D., Baumert, J. H., Goldberg, A. M., Henry, G. W., Kemper, E., and Siegel, M. J. 1988, ApJS, 68, 1
Lasker, B. M., Sturch, C. R., McLean, B. J., Russell, J. L., Jenkner, H., and Shara, M. M. 1990, AJ, 99, 2019

Loveday, J. 1996, MNRAS, 278, 1025

Lutz, R. K. 1980, Comput. J., 23(3), 262

MacGillivray, H. T. and Stobie, R. S. 1984, New results with the COSMOS machine, Vistas Astron., 27, 433

Malagnini, M. L. 1983, A classification algorithm for star-galaxy counts, in ESA SP-201: Statistical Methods in Astronomy, ed. E. J. Rolfe (Noordwijk: ESA), 69

Mirabel, I. F., Dhawan, V., Mignani, R. P., Rodrigues, I., and Guglielmetti, F. 2001, Nature, 413,139

Monet, D. G., Levine, S. E., Canzian, B., Ables, H. D., Bird, A. R., Dahn, C. C., Guetter, H. H., Harris, H. C., Henden, A. A., Leggett, S. K., Levison, H. F., Luginbuhl, C. B., Martini, J., Monet, A. K. B., Munn, J. A., Pier, J. R., Rhodes, A. R., Riepe, B., Sell, S., Stone, R. C., Vrba, F. J., Walker, R. L., Westerhout, G., Brucato, R. J., Reid, I. N., Schoening, W., Hartley, M., Read, M. A., and Tritton, S. B. 2003, AJ, 125,984

Morgan, D. H. 1995, Sky Surveys and Atlases from the Large Schmidt Telescopes, in ASP Conf. Ser. 84, IAU Colloq. 148, The Future Utilisation of Schmidt Telescopes, Ed. J. Chapman, R. Cannon, S. Harrison, and B. Hidayat (San Francisco, CA: ASP), 137

Morrison, J. E., Röser, S., Lasker, B. M., Smart, R. L., and Taff, L. G. 1996, AJ, 111, 1405

Morrison, J. E., Röser, S., McLean, B., Bucciarelli, B., and Lasker, B. 2001, AJ, 121, 1752

Murthy, S. K., Kasif, S., \& Salzberg, S. 1994, arXiv cs/9408103

Myers, J. R., Sande, C. B., Miller, A. C., Warren, J. W. H., and Tracewell, D. A. 2002, SKY2000 Master Catalog, Version 4, ftp://cdsarc.u-strasbg.fr/pub/cats/V/109

Panzera, M. R., Campana, S., Covino, S., Lazzati, D., Mignani, R. P., Moretti, A., and Tagliaferri, G. 2003, A\&A, 399, 351 
Perryman, M. A. C. and ESA 1997, The Hipparcos and Tycho catalogues. Astrometric and photometric star catalogues derived from the ESA Hipparcos Space Astrometry Mission. (ESA SP Series Vol. 1200 ISBN: 9290923997 (set)), (Noordwijk: ESA Publications Division)

Petrosian, A., McLean, B., Allen, R. J., and MacKenty, J. W. 2007, ApJS, 170, 33

Pier, J. R., Munn, J. A., Hindsley, R. B., Hennessy, G. S., Kent, S. M., Lupton, R. H., and Ivezić, Ž. 2003, AJ, 125, 1559

Platais, I., Girard, T. M., Kozhurina-Platais, V., van Altena, W. F., Lopez, C. E., Mendez, R. A., Ma, W.-Z., Yang, T.-G., MacGillivray, H. T., and Yentis, D. J. 2002, SPM Catalog 2.0, VizieR Online Data Catalog I/277

Postman, M., Lubin, L. M., Gunn, J. E., Oke, J. B., Hoessel, J. G., Schneider, D. P., and Christensen, J. A. 1996, AJ, 111, 615

Russell, J. L., Lasker, B. M., McLean, B. J., Sturch, C. R., and Jenkner, H. 1990, AJ, 99, 2059

Sciortino, S., Micela, G., Favata, F., Spagna, A., and Lattanzi, M. G. 2000, A\&A, 357, 460

Skrutskie, M. F., Cutri, R. M., Stiening, R., Weinberg, M. D., Schneider, S., Carpenter, J. M., Beichman, C., Capps, R., Chester, T., Elias, J., Huchra, J., Liebert, J., Lonsdale, C., Monet, D. G., Price, S., Seitzer, P., Jarrett, T., Kirkpatrick, J. D., Gizis, J. E., Howard, E., Evans, T., Fowler, J., Fullmer, L., Hurt, R., Light, R., Kopan, E. L., Marsh, K. A., McCallon, H. L., Tam, R., Van Dyk, S., and Wheelock, S. 2006, AJ, 131, 1163

Spagna, A. 2001, Guide Star Requirements for NGST: Deep NIR Starcounts and Guide Star Catalogs, STScI-NGST-r-0013b Technical Report

Stys, J. and Kriss, G. 2003, The Suitability of Guide Star Catalog 2 (GSC-2) as a Source for JWST Guide Stars, BAAS 202, 410

Taff, L. G., Lattanzi, M. G., and Bucciarelli, B. 1990, ApJ, 358, 359
Thanisch, P. F., M. B. V. and Robin, A. 1984, Image Vis. Comput., 2, 191

Urban, S. E., Corbin, T. E., and Wycoff, G. L. 1998, AJ, 115, 2161

Vallenari, A., Pasetto, S., Bertelli, G., Chiosi, C., Spagna, A., and Lattanzi, M. 2006, A\&A, 451, 125

White, R. L. 1997, Object Classification in Astronomical Images, in Statistical Challenges in Modern Astronomy II ed. G. J. Babu and E. D. Feigelson (Berlin: Springer-Verlag), 135

White, R. L. and Percival, J. W. 1994, Compression and progressive transmission of astronomical images, in Proc. SPIE: Advanced Technology Optical Telescopes V, Vol. 2199, ed. L. M. Stepp, 703

White, R. L., Postman, M., and Lattanzi, M. G. 1992, Compression of the Guide Star Digitised Schmidt Plates, in ASSL: Digitised Optical Sky Surveys, Vol. 174, ed. H. T. MacGillivray, and E. B. Thomson, 167

Zacharias, N., Urban, S. E., Zacharias, M. I., Wycoff, G. L., Hall, D. M., Monet, D. G., and Rafferty, T. J. 2004, AJ, 127, 3043

This 2-column preprint was prepared with the AAS LATEX macros v5.2. 
TABLE 1

Plate MATerial Utilized For the CONSTRUCTion OF THe GSC-II.

\begin{tabular}{|c|c|c|c|c|c|c|c|c|}
\hline $\begin{array}{l}\text { Source }^{\mathrm{a}} \\
\text { Code }\end{array}$ & Survey $^{\mathrm{b}}$ & $\begin{array}{l}\text { Declination } \\
\text { Range }\end{array}$ & Epoch & $\begin{array}{c}\text { Emulsion } \\
+ \text { Filter }\end{array}$ & Band & $\begin{array}{l}\text { Depth } \\
\text { (mag) }\end{array}$ & Fields & $\begin{array}{c}\text { In GSC } \\
2.2 / 2.3\end{array}$ \\
\hline $\mathrm{N}^{(1)}$ & Pal-QV & $\delta \geq 0^{\circ}$ & $1983-85$ & $\mathrm{IIaD}+\mathrm{W} 12$ & $V_{12}$ & 19.5 & 616 & $\mathrm{~N} / \mathrm{Y}$ \\
\hline $\mathrm{S}^{(2)}$ & SERC J & $\delta<-15^{\circ}$ & $1975-87$ & IIIaJ+GG395 & $B_{J}$ & 23.0 & 606 & $\mathrm{Y} / \mathrm{Y}$ \\
\hline $\mathrm{S}^{(2)}$ & SERC EJ & $-15^{\circ}<\delta \leq 0^{\circ}$ & $1979-88$ & IIIaJ+GG395 & $\mathrm{B}_{J}$ & 23.0 & 288 & $\mathrm{Y} / \mathrm{Y}$ \\
\hline $\mathrm{XE}^{(3)}$ & POSS-I E & $\delta \geq-30^{\circ}$ & $1950-58$ & $103 \mathrm{aE}+$ red plexiglass & $E$ & 20.0 & 935 & $\mathrm{~N} / \mathrm{N}$ \\
\hline $\mathrm{XO}$ & POSS-I O & $\delta \geq-30^{\circ}$ & $1950-58$ & $103 \mathrm{aO}$ unfiltered & $O$ & 21.0 & 935 & $\mathrm{~N} / \mathrm{Y}$ \\
\hline XJ & POSS-II J & $\bar{\delta} \geq 0^{\circ}$ & $1987-00$ & IIIaJ+GG385 & $B_{J}$ & 22.5 & 897 & $\mathrm{Y} / \mathrm{Y}$ \\
\hline $\mathrm{XP}$ & POSS-II F & $\delta \geq 0^{\circ}$ & 1987-99 & IIIaF+RG610 & $R_{F}$ & 20.8 & 897 & $\mathrm{Y} / \mathrm{Y}$ \\
\hline XI & POSS-II N & $\delta \geq 0^{\circ}$ & 1989-02 & IV-N +RG9 & $I_{N}$ & 19.5 & 897 & $\mathrm{~N} / \mathrm{Y}$ \\
\hline $\mathrm{XS}$ & AAO-SES & $\delta<-15^{\circ}$ & $1990-00$ & $\mathrm{IIIaF}+\mathrm{OG} 590$ & $R_{F}$ & 22.0 & 606 & $\mathrm{Y} / \mathrm{Y}$ \\
\hline ER & SERC ER & $-15^{\circ}<\delta \leq 0^{\circ}$ & $1990-98$ & IIIaF+OG590 & $R_{F}$ & 22.0 & 288 & $\mathrm{Y} / \mathrm{Y}$ \\
\hline IS & SERC I & $\delta \leq 0^{\circ}$ & $1990-02$ & IV-N + RG715 & $I_{N}$ & 19.5 & 731 & $\mathrm{~N} / \mathrm{Y}$ \\
\hline IS & MW Atlas & $\delta<0^{\circ}$ & $1978-85$ & IV-N + RG715 & $I_{N}$ & 19 & 173 & $\mathrm{~N} / \mathrm{Y}$ \\
\hline $\mathrm{XV}^{(4)}$ & SERC-QV & $-70^{\circ}<\delta \leq 0^{\circ}$ & $1987-88$ & $\mathrm{IIaD}+\mathrm{GG} 495$ & $V_{495}$ & 14 & 94 & $N / Y$ \\
\hline $\mathrm{GR}^{(5)}$ & AAO-SR & $-70^{\circ}<\delta \leq 0^{\circ}$ & 1996-99 & $\mathrm{IIIaF}+\mathrm{OG} 590$ & $R_{F}$ & 20 & 118 & $\mathrm{Y} / \mathrm{Y}$ \\
\hline
\end{tabular}

${ }^{a}$ The source code used by the GSC-II database to identify individual plates. For example, XJ442, XP442, and XI442 identity the blue, red, and near-infrared plates of the 442th POSS-II field.

${ }^{\mathrm{b}}$ Survey-filter abbreviations: see NOTE below.

${ }^{1}$ The Pal-QV survey was taken specifically for GSC-I. Schott filter GG495 replaced W12 starting 3 June 1984 . Scanned with 25 micron sampling $(1.7 \mathrm{arcsec} / \mathrm{pixel})$.

${ }^{2} \mathrm{~S}$ plates originally scanned with 25 micron sampling, 440 plates at lower latitudes rescanned with 15 micron sampling (1.0 $\mathrm{arcsec} / \mathrm{pixel})$.

${ }^{3}$ POSS-I E filter was red plexiglass no. 2444; scanned at 25 micron sampling except 123 plates scanned at 15 micron, mostly south of $-18^{\circ}$.

${ }^{4}$ SERC "Quick V" survey taken specifically for GSC-I with 4-min exposures to cover crowded southern Milky Way fields $\left(|b|<15^{\circ},-112^{\circ}<l<34^{\circ}\right.$ plus two plates on the LMC). (Three similar "XX" plates were centered on M31 and each of the Magellanic Clouds.)

${ }^{5}$ AAO-SR survey taken specifically for GSC-II with 5-min exposures to cover crowded southern Milky Way fields $\left(|b|<15^{\circ},-112^{\circ}<l<34^{\circ}\right.$ plus two plates on the LMC).

Note.-Here, Pal-QV refers to the Palomar "Quick V" survey (Lasker et al. (1990)), SERC refers to the Science \& Engineering Research Council surveys, POSS I and II are the Palomar Observatory Sky Surveys I and II, AAO-SES and -SR refer to the Anglo-Australian Observatory Second Epoch Survey and Short-Red survey, and the MW Atlas is the SERC I/SR Atlas of the Milky Way and Magellanic Clouds (ref?). POSS-I and Pal-QV surveys utilized a $6^{\circ}$ grid of telescope pointings, with minimum plate overlap, while the other surveys used a $5^{\circ}$ degree grid. Unless stated otherwise, all plates scanned at 15 micron sampling ( 1 arcsec/pixel). Scans performed on glass copies of the S, XE and XO plates, rather than on the original plates.

TABLE 2

Classification PARAmEters.

\begin{tabular}{cc}
\hline \hline Parameter & Definition \\
\hline 1 & Integrated density \\
2 & Peak density \\
3 & Semimajor axis \\
4 & Semiminor axis \\
5 & Ellipticity \\
$6,7,8$ & $\sigma_{x}^{2}, \sigma_{y}^{2}, \sigma_{x y}^{2}$ \\
$9-12$ & Texture features (Malagnini $\left.\frac{(1983)}{(1990)}\right)$ \\
$13-14$ & Spike features (Lasker et al. \\
$15-30$ & Area at 16 detection thresholds \\
\hline
\end{tabular}


TABLE 3

GSC 2.3 (July 2006). Export Binary Table Fields and Types

\begin{tabular}{|c|c|c|c|c|}
\hline Number & Field name & Format & Unit & Notes \\
\hline 1 & gscID2 & Integer*4 $(\mathrm{J})$ & id & GSC 2.3 object id \\
\hline 2 & gsc1ID & Character*11 (11A) & id & GSC 1.1 object id \\
\hline 3 & hstID & Character*11 (11A) & id & $H S T$ object id \\
\hline 4 & RightAsc & Real*8 (D) & $\mathrm{rad}$ & Right ascension ICRF \\
\hline 5 & Declination & Real*8 (D) & $\mathrm{rad}$ & Declination ICRF \\
\hline 6 & PositionEpoch & $\mathrm{Real}^{*} 4(\mathrm{E})$ & year & Position epoch ${ }^{1}$ \\
\hline 7 & raEpsilon & Real*4 (E) & $\operatorname{arcsec}$ & Reference error on R.A. ${ }^{2}$ \\
\hline 8 & decEpsilon & Real*4 $^{*}(\mathrm{E})$ & $\operatorname{arcsec}$ & Reference error on $\mathrm{DEC}^{2}$ \\
\hline 14 & FpgMag & Real*4 $^{*}(\mathrm{E})$ & mag & $R_{F}$ photographic magnitude \\
\hline 15 & FpgMagErr & Real*4 $^{*}(\mathrm{E})$ & mag & Reference error on $R_{F}^{2}$ \\
\hline 16 & FpgMagCode & Integer*2 $^{*}(\mathrm{I})$ & - & Filter code of $R_{F}$ \\
\hline 17 & JpgMag & Real*4 $(\mathrm{E})$ & mag & $B_{J}$ Photographic magnitude \\
\hline 18 & JpgMagErr & Real*4 $^{*}(\mathrm{E})$ & mag & Reference error on $B_{J}$ \\
\hline 19 & JpgMagCode & Integer*2 $^{*}(\mathrm{I})$ & - & Filter code of $B_{J}$ \\
\hline 20 & VMag & Real*4 (E) & mag & $V$ [photographic] magnitude 3 \\
\hline 21 & VMagErr & $\operatorname{Real}^{*} 4(\mathrm{E})$ & mag & Reference error on $V^{2}$ \\
\hline 22 & VMagCode & Integer*2 $^{*}(\mathrm{I})$ & - & Filter code of $V$ \\
\hline 23 & NpgMag & Real*4 (E) & mag & $I_{N}$ photographic magnitude \\
\hline 24 & NpgMagErr & Real*4 $^{*}(\mathrm{E})$ & mag & Reference error on $I_{N}^{2}$ \\
\hline 25 & NpgMagCode & Integer*2 $(\mathrm{I})$ & - & Filter code of $I_{N}$ \\
\hline 29 & BMag & Real*4 (E) & mag & $B$ Magnitude $^{4}$ \\
\hline 30 & BMagErr & $\operatorname{Real}^{*} 4(\mathrm{E})$ & mag & Reference error on $B$ \\
\hline 31 & BMagCode & Integer*2 $(\mathrm{I})$ & - & Filter code of $B$ \\
\hline 47 & classification & Integer*4 $(\mathrm{J})$ & - & Morphological classification 5 \\
\hline 48 & semiMajorAxis & Real*4 (E) & pixels & Image semimajor axis ${ }^{6}$ \\
\hline 49 & eccentricity & Real*4 (E) & - & Image eccentricity ${ }^{6}$ \\
\hline 50 & positionangle & Real*4 (E) & degrees & Image orientation 6 \\
\hline 51 & sourceStatus & Integer*4 $(\mathrm{J})$ & - & Object processing status flag \\
\hline
\end{tabular}

${ }^{1}$ Plate epoch for GSC-II objects. For Tycho 2 objects, for which $T_{\alpha} \neq T_{\delta}$, the R.A. epoch is given.

${ }^{2}$ These astrometric and photometric errors are not formal statistical uncertainties but a raw and conservative estimates to be used for telescope operations.

${ }^{3}$ This field may include: (a) photographic $V_{12}$ or $V_{495}$ from IIaD plates, (b) $V_{T}$ of Tycho-2 stars, or (c) Johnson $V$ from SKY2000.

${ }^{4} B_{T}$ of Tycho-2 stars or Johnson B from SKY2000 or photographic O from POSS-I

${ }^{5}$ Image classification: $0=$ "star" (i.e. point-like object) and $3=$ "nonstar" (i.e. extended object).

${ }^{6}$ Morphological parameters of the same image used for the position. 
TABLE 4

GSC 2.3 BANDPASS CODES.

\begin{tabular}{rllr}
\hline \hline Code & Band & Emulsion+Filter & Survey/Catalog \\
\hline 0 & $B_{J}$ & IIIaJ + GG395 & SERC-J, SERC-EJ \\
1 & $V_{12}$ & IIaD + W12 & Pal-QV \\
3 & $\mathrm{~B}$ & Johnson B & SKY2000 \\
4 & $\mathrm{~V}$ & Johnson V & SKY2000 \\
5 & $R_{F}$ & IIIaF + RG630 & ESO-R \\
6 & $V_{495}$ & IIaD + GG495 & Pal-QV, SERC-SV \\
7 & $\mathrm{O}$ & 103aO unfiltered & POSS-I O \\
18 & $B_{J}$ & IIIaJ + GG385 & POSS-II J \\
35 & $R_{F}$ & IIIaF + RG610 & POSS-II F \\
36 & $R_{F}$ & IIIaF + OG590 & SERC-ER, SERC-SR, \\
& & & AAO-R, AAO-GR \\
37 & $I_{N}$ & IV-N + RG9 & POSS-II IR \\
38 & $I_{N}$ & IV-N + RG715 & SERC-IR \\
41 & $B_{T}$ & & Tycho B \\
42 & $V_{T}$ & & Tycho V \\
\hline
\end{tabular}

TABLE 5

Source Status Flag Codes.

\begin{tabular}{lc}
\hline \hline Digits $\left({ }^{*}\right)$ & Flag Meaning \\
\hline 1 and 2 & Number of observations, some observations maybe excluded from GSC 2.3. \\
3 & Centroider type used: $0=$ barycenter, $1=$ circular ${ }^{\mathrm{a}}, 2=$ elliptical, \\
& $3=\mathrm{FPA}+$ applied barycenter $\mathrm{b}, 4=$ multicircular, \\
& $5=$ multielliptical, $6=\mathrm{FPA}+$ circular, $7 \mathrm{FPA}+$ elliptical \\
4 & Quality of exported $R_{F}$ magnitude: $0=$ not present $1=$ fit, $2=$ extrapolated \\
5 & Quality of exported $B_{J}$ magnitude: $0=$ not present, $1=$ fit, $2=$ extrapolated \\
6 & Quality of exported $V$ magnitude: $0=$ not present, $1=$ fit, $2=$ extrapolated \\
7 & Classification unanimity: $0=$ mixed vote, $1=$ unanimous vote, $3=$ unanimous artifact \\
8 & Classification voters: $0=$ multiple 15 um scans, $1=$ One 15 um scan, \\
& $2=$ multiple 25 um scans, $3=$ One 25 um scan \\
9 & Processing status: $0=$ completed processing, \\
& $1=$ object too big to be cut out on at least one plate \\
10 & Deblended object: $0=$ single object on all plates, \\
& $1=$ child (deblended) object on at least one plate \\
\hline
\end{tabular}

*counting from right to left; flags for Tycho and SKYMAP objects are 99999900 and 88888800 respectively

${ }^{a}$ All non barycentric centroiding using Gaussian fits

${ }^{\mathrm{b}} \mathrm{FPA}=$ Fractional Pixel Allocation 
TABLE 6

GSC 2.3 GLOBAL Statistics

\begin{tabular}{rrrr}
\hline \hline & $\begin{array}{c}\text { Northern } \\
\text { hemisphere }\end{array}$ & $\begin{array}{c}\text { Southern } \\
\text { hemisphere }\end{array}$ & \multicolumn{1}{c}{$\begin{array}{c}\text { All } \\
\text { sky }\end{array}$} \\
\hline $\begin{array}{r}\text { Objects } \\
396700598\end{array}$ & 548892085 & $\mathbf{9 4 5 5 9 2 6 8 3}$ \\
Point-like objects & 88481909 & 118206935 & 206688844 \\
Extended objects & 308218689 & 430685150 & 738903839 \\
\hline$R_{F}$ magnitudes & 354300890 & 452765492 & 807066382 \\
$B_{J}$ magnitudes & 307949064 & 403822005 & 711771069 \\
$V_{\text {pg magnitudes }}$ & 154786490 & 67947515 & 222734005 \\
$I_{N}$ magnitudes & 245102118 & 329725734 & 574827852 \\
$B_{J}, R_{F}$, and $I_{N}$ magnitudes & 275035930 & 330759735 & 605795665 \\
& 210601441 & 236097358 & 446668799 \\
\hline GSC 1.1 objects & 9071325 & 9598246 & 18669571 \\
Tycho 2 stars ${ }^{1}$ & 1201897 & 1321635 & 2523552 \\
Sky2000/Skymap ${ }^{1}$ & 348458 & 190364 & 473943 \\
\hline
\end{tabular}

\footnotetext{
${ }^{1}$ Without double/multiple entries (see explanation in section 4.2): 2,519,152 Tycho-
} 2 stars and 175,632 SKY2000 stars 
TABLE 7

GSC 2.3 (July 2006). All-Sky Cumulative COUnts ${ }^{1}$.

\begin{tabular}{|c|c|c|c|c|}
\hline mag & $\mathrm{N}_{\mathrm{obj}}\left(B_{J}\right)$ & $\mathrm{N}_{\mathrm{obj}}\left(R_{F}\right)$ & $\mathrm{N}_{\mathrm{obj}}\left(I_{N}\right)$ & $\mathrm{N}_{\mathrm{obj}}\left(B_{J}+R_{F}+I_{N}\right)^{2}$ \\
\hline 10.0 & 20715 & 35949 & 192984 & 50794 \\
\hline 10.5 & 34491 & 82556 & 443489 & 175119 \\
\hline 11.0 & 54584 & 239047 & 1010629 & 564997 \\
\hline 11.5 & 86600 & 706719 & 2190201 & 1519395 \\
\hline 12.0 & 154228 & 1829779 & 4415724 & 3367609 \\
\hline 12.5 & 364579 & 3994288 & 8089061 & 6305430 \\
\hline 13.0 & 1060240 & 7458931 & 13612182 & 10657925 \\
\hline 13.5 & 2603441 & 12638946 & 21619677 & 16943348 \\
\hline 14.0 & 5056922 & 20196571 & 32990022 & 25837448 \\
\hline 14.5 & 8668396 & 31041175 & 48953930 & 38151152 \\
\hline 15.0 & 13898051 & 46366494 & 71017843 & 54914864 \\
\hline 15.5 & 21361566 & 67781378 & 101286676 & 77348928 \\
\hline 16.0 & 31792178 & 97002324 & 142016145 & 107002624 \\
\hline 16.5 & 46052408 & 135717387 & 196139738 & 145725200 \\
\hline 17.0 & 65119781 & 186270427 & 267399004 & 195532864 \\
\hline 17.5 & 90046889 & 250652663 & 349775619 & 254950176 \\
\hline 18.0 & 121721348 & 327112112 & 434137985 & 319880384 \\
\hline 18.5 & 161745847 & 405027755 & 510834052 & 383714272 \\
\hline 19.0 & 210802304 & 489823020 & 562417267 & 427113344 \\
\hline 19.5 & 270784037 & 583790690 & 574291825 & 443187232 \\
\hline 20.0 & 340876792 & 685566332 & 574700787 & 446393632 \\
\hline 20.5 & 417992984 & 783784283 & 574823790 & 446588640 \\
\hline 21.0 & 497409326 & 805951305 & 574827840 & 446645408 \\
\hline 21.5 & 573255946 & 806469272 & 574827852 & 446675616 \\
\hline 22.0 & 649675534 & 806698629 & 574827852 & 446689312 \\
\hline 22.5 & 707695255 & 806828565 & 574827852 & 446696416 \\
\hline 23.0 & 711708557 & 806937362 & 574827852 & 446698848 \\
\hline 23.5 & 711722103 & 807017618 & 574827852 & 446699680 \\
\hline 24.0 & 711736633 & 807061276 & 574827852 & 446699680 \\
\hline all & 711771069 & 807066382 & 574827852 & 466699774 \\
\hline
\end{tabular}

\footnotetext{
${ }^{1}$ Cumulative counts from 0 magnitude to $m$ given in the first column

${ }^{2}$ The number of objects with computed photometry in the 3 passbands are accumulated according to magnitude steps in $R_{F}$
} 
TABLE 8

GSC 2.3 - UCAC 2/SDSS: Astrometric Residuals.

\begin{tabular}{|c|c|c|c|c|c|c|c|c|c|c|}
\hline \multirow[b]{2}{*}{$\begin{array}{c}R_{F} \\
\text { (mag) }\end{array}$} & \multicolumn{5}{|c|}{ stellar objects } & \multicolumn{5}{|c|}{ extended objects } \\
\hline & $N_{\text {star }}$ & $\begin{array}{c}\sigma_{\Delta \alpha} \\
\left({ }^{\prime \prime}\right)\end{array}$ & $\begin{array}{r}\epsilon_{\Delta \alpha} \\
\left({ }^{\prime \prime}\right)\end{array}$ & $\begin{array}{r}\sigma_{\Delta \delta} \\
\left({ }^{\prime \prime}\right)\end{array}$ & $\begin{array}{l}\epsilon_{\Delta \delta} \\
\left({ }^{\prime \prime}\right)\end{array}$ & $N_{\text {ext }}$ & $\begin{array}{c}\sigma_{\Delta \alpha} \\
\left({ }^{\prime \prime}\right)\end{array}$ & $\begin{array}{r}\epsilon_{\Delta \alpha} \\
\left({ }^{\prime \prime}\right)\end{array}$ & $\begin{array}{r}\sigma_{\Delta \delta} \\
\left({ }^{\prime \prime}\right)\end{array}$ & $\begin{array}{l}\epsilon_{\Delta \delta} \\
\left({ }^{\prime \prime}\right)\end{array}$ \\
\hline \multicolumn{11}{|l|}{ UCAC 2} \\
\hline $11.5-12.0$ & 786859 & 0.16 & 0.20 & 0.17 & 0.20 & 81641 & 0.63 & 0.67 & 0.59 & 0.63 \\
\hline $12.5-13.0$ & 2688254 & 0.16 & 0.19 & 0.16 & 0.19 & 438091 & 0.57 & 0.62 & 0.53 & 0.58 \\
\hline $13.5-14.0$ & 5467031 & 0.14 & 0.19 & 0.14 & 0.18 & 1559410 & 0.55 & 0.60 & 0.52 & 0.57 \\
\hline $14.5-15.0$ & 9741299 & 0.13 & 0.19 & 0.13 & 0.17 & 4559916 & 0.48 & 0.54 & 0.46 & 0.51 \\
\hline $\begin{array}{c}15.5-16.0 \\
\text { SDSS }\end{array}$ & 16289992 & 0.12 & 0.20 & 0.13 & 0.18 & 9553274 & 0.42 & 0.48 & 0.40 & 0.45 \\
\hline $14.5-15.0$ & 566319 & 0.14 & 0.21 & 0.14 & 0.16 & 37169 & 0.21 & 0.27 & 0.21 & 0.24 \\
\hline $15.5-16.0$ & 878746 & 0.13 & 0.21 & 0.13 & 0.16 & 115608 & 0.22 & 0.28 & 0.22 & 0.25 \\
\hline $16.5-17.0$ & 1194037 & 0.13 & 0.22 & 0.13 & 0.16 & 394648 & 0.18 & 0.25 & 0.18 & 0.21 \\
\hline $17.5-18.0$ & 1528006 & 0.14 & 0.23 & 0.14 & 0.17 & 1011007 & 0.18 & 0.25 & 0.18 & 0.21 \\
\hline $18.5-19.0$ & 1896983 & 0.16 & 0.26 & 0.16 & 0.20 & 2759341 & 0.21 & 0.28 & 0.21 & 0.24 \\
\hline 19.5-20.0 & 1608896 & 0.23 & 0.31 & 0.23 & 0.25 & 7965088 & 0.32 & 0.37 & 0.31 & 0.34 \\
\hline
\end{tabular}

TABLE 9

GPSC-2 - GSC 2.3 PHOTOMETRIC RESIDUALS

\begin{tabular}{lrrrrrr}
\hline \hline \multicolumn{1}{c}{ Objects } & \multicolumn{3}{c}{ Northern Hemisphere } & \multicolumn{3}{c}{ Southern Hemisphere } \\
& Nbr & $\left\langle\Delta R_{F}\right\rangle$ & $\sigma_{\Delta R_{F}}$ & \multicolumn{1}{c}{ Nbr } & $\left\langle\Delta R_{F}\right\rangle$ & $\sigma_{\Delta R_{F}}$ \\
\hline All objects & 204322 & 0.01 & 0.28 & 175364 & 0.04 & 0.33 \\
Stellar objects & 84996 & -0.03 & 0.18 & 60613 & -0.02 & 0.25 \\
All objects (high latitude) & 36714 & 0.03 & 0.18 & 23977 & 0.02 & 0.22 \\
Stellar objects (high latutude) & 25508 & -0.01 & 0.13 & 12846 & -0.02 & 0.16 \\
\hline
\end{tabular}

TABLE 10

GSC 2.3 - SDSS PHOtOMETRIC RESIDUALS $\Delta B_{\mathrm{J}}$

\begin{tabular}{crrrr}
\hline \hline & \multicolumn{2}{c}{ North } & \multicolumn{2}{c}{ South } \\
\multicolumn{1}{c}{$B_{\mathrm{J}}$} & \multicolumn{1}{c}{$\mathrm{n}$} & \multicolumn{1}{c}{$\sigma_{\Delta B_{\mathrm{J}}}$} & \multicolumn{1}{c}{$\mathrm{n}$} & $\sigma_{\Delta B_{\mathrm{J}}}$ \\
\hline $14.5-15.0$ & 44333 & 0.42 & 23713 & 0.31 \\
$15.5-16.0$ & 83030 & 0.19 & 47614 & 0.14 \\
$16.5-17.0$ & 129263 & 0.17 & 76672 & 0.14 \\
$17.5-18.9$ & 167603 & 0.17 & 102888 & 0.17 \\
$18.5-19.0$ & 203192 & 0.16 & 114972 & 0.19 \\
$19.5-20.0$ & 246944 & 0.18 & 118327 & 0.18 \\
$20.5-21.0$ & 263612 & 0.22 & 109137 & 0.22 \\
21.5 .22 .0 & 150603 & 0.27 & 58421 & 0.27 \\
\hline
\end{tabular}


TABLE 11

GSC 2.3 - SDSS PHOtometric RESIDUALS $\Delta R_{\mathrm{F}}$

\begin{tabular}{crrrr}
\hline \hline & \multicolumn{2}{c}{ North } & \multicolumn{2}{c}{ South } \\
$R_{\mathrm{F}}$ & \multicolumn{1}{c}{$\mathrm{N}$} & $\sigma_{\Delta R_{\mathrm{F}}}$ & \multicolumn{1}{c}{$\mathrm{N}$} & $\sigma_{\Delta R_{\mathrm{F}}}$ \\
\hline $13.5-14.0$ & 49732 & 0.16 & 26838 & 0.17 \\
$14.5-15.0$ & 91999 & 0.13 & 52713 & 0.12 \\
$15.5-16.0$ & 146229 & 0.12 & 84616 & 0.11 \\
$16.5-17.0$ & 198843 & 0.12 & 117889 & 0.13 \\
$17.5-18.0$ & 254016 & 0.13 & 140616 & 0.13 \\
$18.5-19.0$ & 306114 & 0.15 & 143052 & 0.15 \\
$19.5-20.0$ & 222294 & 0.22 & 88951 & 0.21 \\
\hline
\end{tabular}

Table 12

GSC 2.3 - SDSS PHOtOMETRIC RESIDUALS $\Delta I_{\mathrm{N}}$

\begin{tabular}{crrrr}
\hline \hline & \multicolumn{2}{c}{ North } & \multicolumn{2}{c}{ South } \\
$I_{\mathrm{N}}$ & \multicolumn{1}{c}{$\mathrm{N}$} & $\sigma_{\Delta I_{\mathrm{N}}}$ & \multicolumn{1}{c}{$\mathrm{N}$} & $\sigma_{\Delta I_{\mathrm{N}}}$ \\
\hline $13.5-14.0$ & 56684 & 0.17 & 34795 & 0.12 \\
$14.5-15.0$ & 120778 & 0.13 & 66884 & 0.10 \\
$15.5-16.0$ & 186497 & 0.14 & 106972 & 0.11 \\
$16.5-17.0$ & 258430 & 0.15 & 150240 & 0.13 \\
$17.5-18.0$ & 308639 & 0.19 & 164387 & 0.21 \\
$18.5-19.0$ & 143655 & 0.26 & 59368 & 0.25 \\
\hline
\end{tabular}


TABLE 13

GSC II SPECIFICATIONS ${ }^{\mathrm{a}}$ AND PERFORMANCE $\left(V_{\text {lim }}=18\right)$

\begin{tabular}{|c|c|c|}
\hline & Specifications & Performance \\
\hline \multicolumn{3}{|l|}{ Astrometry } \\
\hline Reference frame & ICRF & ICRF \\
\hline Absolute position error & $<0 . " 5$ & $<0 . " 3 \mathrm{~b}$ \\
\hline Relative position error over a $0 .^{\circ} 5$ field & $\leq 0 .{ }^{*} 2$ & $<0 . " 2 \mathrm{~b}$ \\
\hline Adherence to reference frame & $<0 . " 15$ & $<0 . " 15^{\mathrm{c}}$ \\
\hline Proper motion error (total) & $<0.004$ " year $^{-1}$ & Not released \\
\hline \multicolumn{3}{|l|}{ Photometry } \\
\hline Passbands & At least 2 (1 color $)$ & $B_{J}, R_{F}, I_{N}$, Some $V$ and $O$ \\
\hline Magnitude error & $0.1-0.2 \mathrm{mag}$ & $<0.2$ \\
\hline Completeness to magnitude limit & Yes & For $90 \%$ of the sky ${ }^{d}$ \\
\hline Stellar classification: & $95 \%$ & $98 \%$ e \\
\hline
\end{tabular}

${ }^{a}$ source: GSC-II Implementation Plan (Lasker and Lattanzi 1994). Performance statistics are given for objects to magnitude limit $\mathrm{V}=18$, as in the original implementation plan

${ }^{\mathrm{b}}$ From a global comparison to DR5 of the SDSS, for objects classified as stellar. The random contribution for extended sources is approximately $20 \%$ worse

${ }^{\mathrm{c}}$ Averaged over all plates, all magnitudes

${ }^{\mathrm{d}}$ Completeness is reached outside of the galactic plane; for details see Section 5.6

e $98 \%$ of objects classified as stars are confirmed as stellar by a comparison to SDSS; see Section 5.5 for details 
TABLE 14

GSC 2.3 Global PRoperties

\begin{tabular}{lcc}
\hline \hline & & \\
\hline Total objects & $945,592,683$ & \\
Magnitude limit & $B_{J}=22.5, R_{F}=20.5$, and $I_{N}=19.5$ & \\
Mean epoch of positions & 1992.5 & \\
Reference frame & ICRF & \\
Astrometric reference catalogs & ACT + Tycho- $~$ & Extended objects \\
Average positional accuracy $(a)$ & Stellar objects & $0 . " 35$ \\
$R_{F}<18.5$ & $0 . " 28$ & $0 . " 37$ \\
$18.5 \leq R_{F} \leq 19.5$ & $0 . " 30$ & $0 . " 50$ \\
$R_{F}>19.5$ & $0 . " 40$ & Extended objects \\
Average positional precision ${ }^{(b)}$ & Stellar objects & $0 . " 25$ \\
$R_{F}<18.5$ & $0 . " 20$ & $0 . " 30$ \\
$18.5 \leq R_{F} \leq 19.5$ & $0 . " 22$ & $0 . " 44$ \\
$R_{F}>19.5$ & $0 . " 32$ & \\
Photometric reference catalogs & GSPC2 + Tycho \\
Average photometric accuracy(stellar sources) $)^{(c)}$ & & \\
$R_{F}<18.5$ & 0.13 mag & \\
$18.5 \leq R_{F} \leq 19.5$ & 0.15 mag & Up to $R_{F}=20, l>30^{\circ}(d)$ \\
$R_{F}>19.5$ & $0.22 \mathrm{mag}$ & \\
Completeness & $>98 \%$ & \\
\hline
\end{tabular}

${ }^{(a)}$ Accuracy referes to the combined contribution of random and systematic errors. The values are global averages for the SDSS DR5 sample. Note that plate-to-plate as well as north-south discrepancies can reach a few tenths of an arcsecond. This is mainly due to residual systematic effects, as described in Section 5.2, which will be addressed in the next catalog release.

${ }^{(b)}$ These values, estimated using formula 5 , only show the random part contribution to the positional error.

${ }^{(c)}$ Based on the SDSS DR5 sample. Systematic offsets of the order few hundredths of a magnitude; photometry of non-stellar sources suffers from systematic errors which, for very bright objects, can be as high as $\approx 2$ mag, see Fig. 26.

${ }^{(d)}$ For the Galactic plane, the fraction of sky with less than $90 \%$ completeness limit is shown in Fig. 32 as function of magnitude. 